Aus dem Institut für Diagnostische und Interventionelle Radiologie

(Prof. Dr. med. J. Lotz)

der Medizinischen Fakultät der Universität Göttingen

\title{
Evaluation eines Echtzeit-Verfahrens in der kardialen Magnetresonanztomographie bei Patienten mit Herzrhythmusstörungen am Beispiel von Vorhofflimmern
}

\author{
INAUGURAL-DISSERTATION \\ zur Erlangung des Doktorgrades \\ der Medizinischen Fakultät der \\ Georg-August-Universität zu Göttingen
}

vorgelegt von

Eckhart Thassilo von Loesch

aus

Kassel

Göttingen 2016 
Diese Arbeit wurde im Rahmen der interdisziplinären Arbeitsgruppe kardiovaskuläre Bildgebung der UMG (Leitung: Prof. Dr. med. J. Lotz) angefertigt, unterstützt und betreut.

Dekan:

Referent/in

Ko-Referent/in:

Drittreferent/in:
Prof. Dr. rer. nat. H. K. Kroemer

Prof. Dr. med. C. Unterberg-Buchwald

Prof. Dr. med. Matthias Sigler

Datum der mündlichen Prüfung: 09.03.2017 
Hiermit erkläre ich, die Dissertation mit dem Titel „Evaluation eines Echtzeit-Verfahrens in der kardialen Magnetresonanztomographie bei Patienten mit Herzrhythmusstörungen am Beispiel von Vorhofflimmern" eigenständig angefertigt und keine anderen als die von mir angegebenen Quellen und Hilfsmittel verwendet zu haben.

Göttingen, den 


\section{Inhaltsverzeichnis}

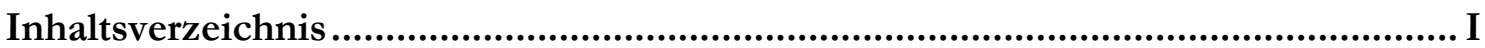

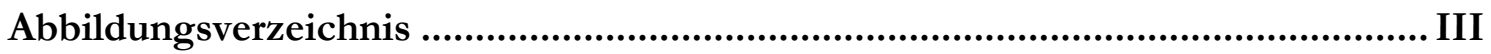

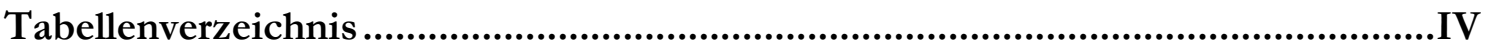

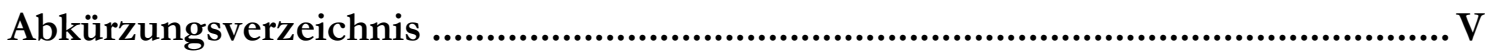

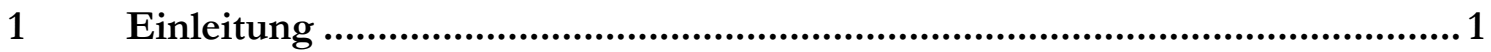

$1.1 \quad$ Stellenwert der MRT in der Herzbildgebung .......................................................................... 1

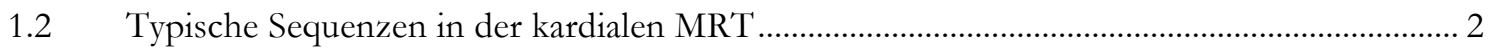

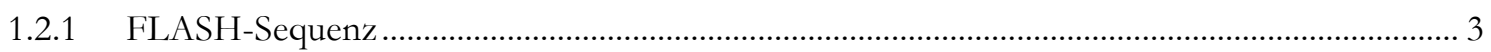

1.2.2 Balanced-steady-state-free-precession-(bSSFP)-Sequenz ...................................................... 3

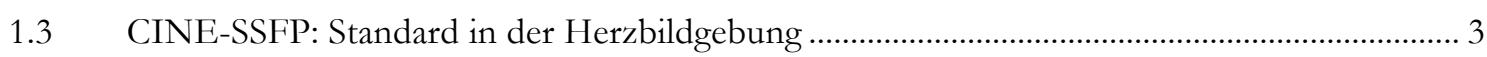

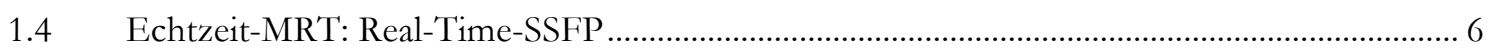

1.5 Kardiale MRT bei Patienten mit Arrhythmien ....................................................................... 7

1.6 Definition des Krankheitsbildes Vorhofflimmern (VHF) …….............................................. 8

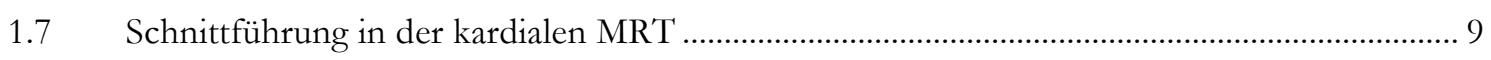

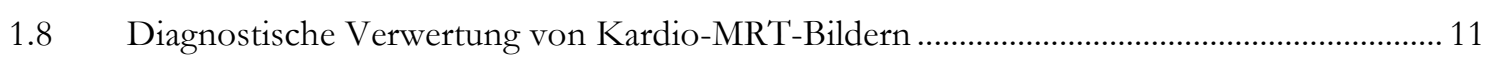

1.9 Vergleichsmethode: Transthorakale Echokardiographie ............................................................ 12

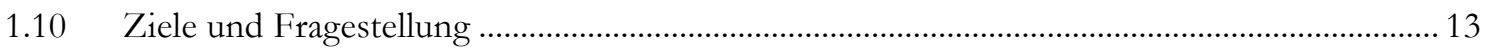

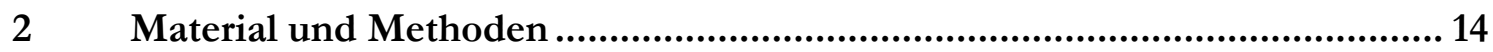

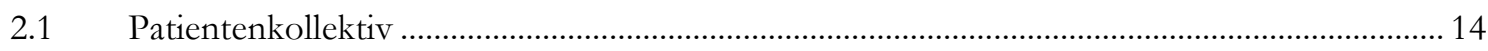

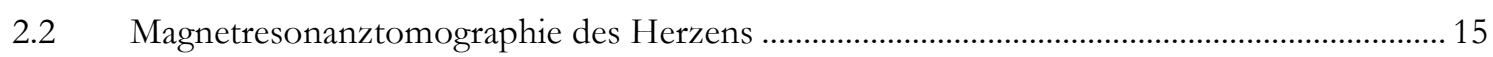

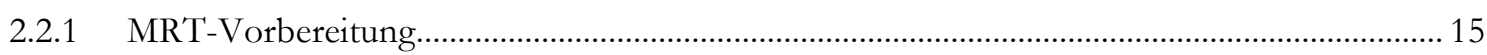

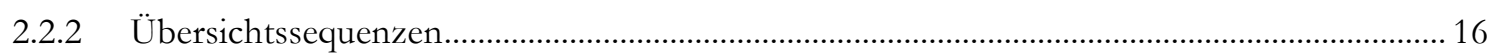

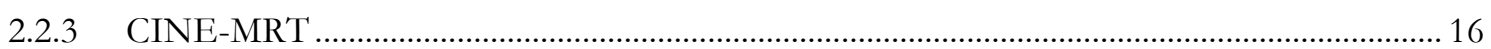

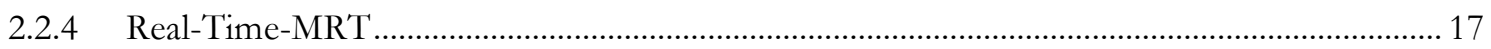

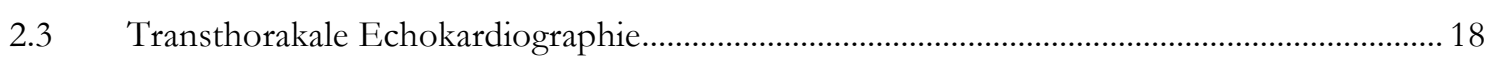

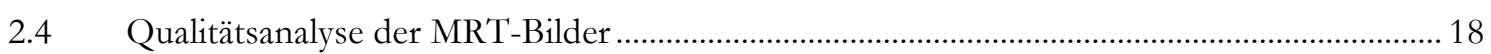

2.5 Analyse der linksventrikulären Funktion anhand der MRT-Aufnahmen ................................ 19

2.6 Analyse der linksventrikulären Funktion anhand der Echokardiographie ............................... 24

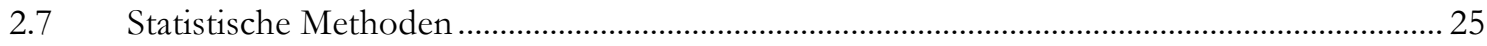

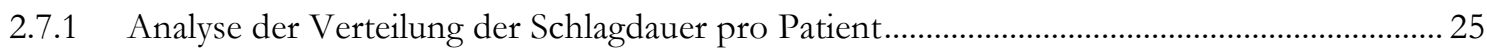

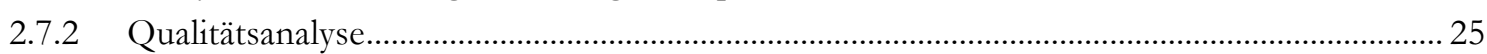

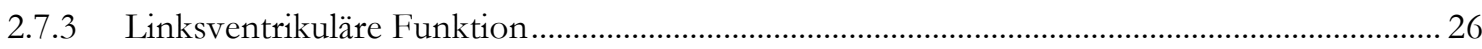

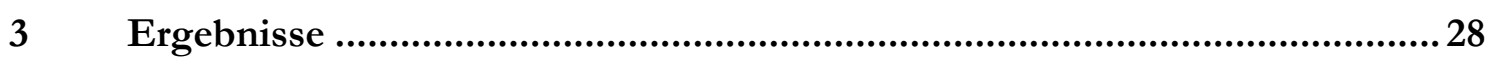

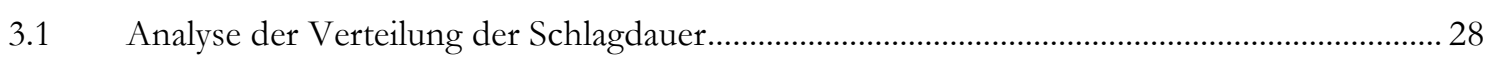




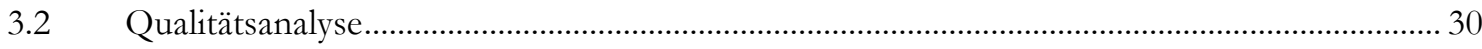

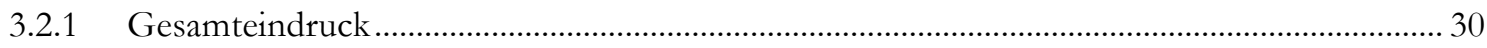

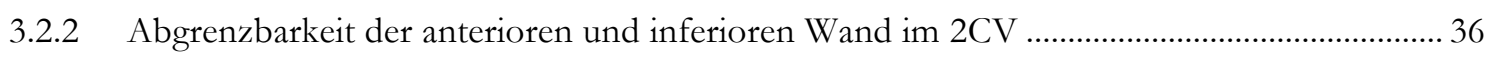

3.2.3 Abgrenzbarkeit des Septums und der Seitenwand ….......................................................... 36

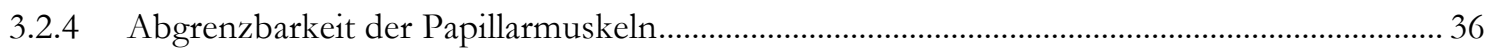

3.2.5 Interrater-Variabilität und Intrarater-Variabilität.................................................................... 42

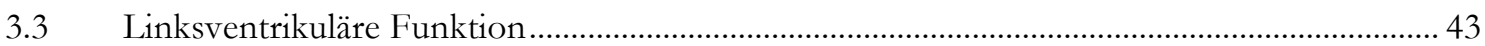

3.3.1 Vergleich der linksventrikulären Funktionsparameter in CINE-SSFP und Real-Time-

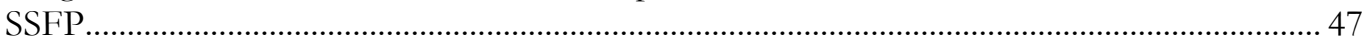

3.3.2 Vergleich der linksventrikulären Funktionsparameter in CINE-SSFP, Real-Time-SSFP und Echokardiographie ..................................................................................................... 50

3.3.3 Vergleich der Mittelung über sieben, fünf und drei Schläge des Real-Time-SSFP-4CV ....... 51

3.3.4 Schlag-zu-Schlag-Analyse im Real-Time-SSFP-4CV .......................................................... 53

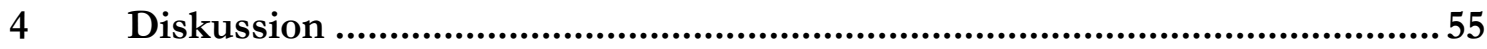

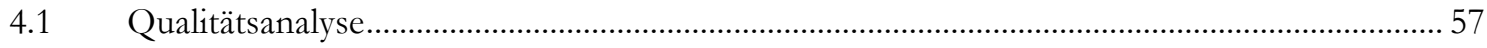

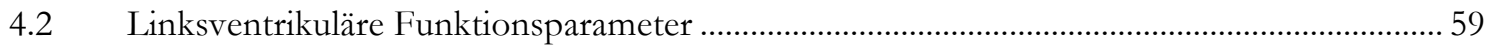

4.2.1 Vergleich der Mittelung über sieben, fünf und drei Schläge des Real-Time-SSFP-4CV .......60

4.2.2 Schlag-zu-Schlag-Analyse im Real-Time-SSFP-4CV ............................................................ 61

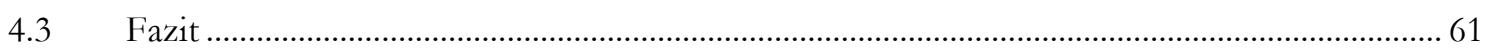

$5 \quad$ Zusammenfassung............................................................................................. 63

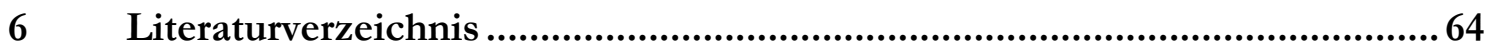




\section{Abbildungsverzeichnis}

Abbildung 1: Prinzip der CINE-SSPP - prospektives Triggern und retrospektives Gating. ............ 5

Abbildung 2: Graphische Darstellung des Prinzips der Real-Time-Datenakquisition. ....................... 7

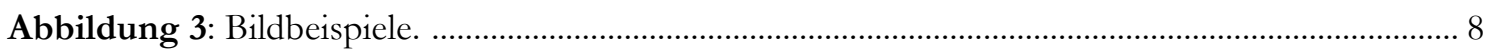

Abbildung 4: Schnittführung in der kardialen MRT. ....................................................................... 10

Abbildung 5: Segmentation mit QMass am Beispiel eines Bildes aus dem CINE-SSFP-SA-

Stapel und eines Real-Time-SSFP-4CV. ............................................................. 12

Abbildung 6: Vorbereitung eines Patienten auf die MRT-Untersuchung. ...................................... 15

Abbildung 7: Übersicht über das MRT-Untersuchungsprotokoll. ...................................................... 16

Abbildung 8: Segmentation der CINE-SSFP-Aufnahmen mit QMass. .......................................... 21

Abbildung 9: Segmentation der Real-Time-SSFP mit QMass. ........................................................ 23

Abbildung 10: Echokardiographie - Segmentation enddiastolisch und endsystolisch. .................... 24

Abbildung 11: Beispiel für einen Bubble-Plot. ................................................................................... 26

Abbildung 12: Histogramme zur Darstellung der Verteilung der Schlagdauer. ............................... 28

Abbildung 13: Bubble-Plots für die Qualitätsanalyse der langen Achsen im Gesamteindruck. ...... 31

Abbildung 14: Bubbleplots für die Qualitätsanalyse der kurzen Achsen im Gesamteindruck. ....... 32

Abbildung 15: Intraindividueller Vergleich CINE- und Real-Time-SSFP-2CV und -3CV. ............33

Abbildung 16: Intraindividueller Vergleich CINE- und Real-Time-SSFP-4CV. .............................. 34

Abbildung 17: Intraindividueller Vergleich CINE- und Real-Time-SSFP-SA-mid. .......................... 35

Abbildung 18: Bubble-Plots für Qualitätsanalyse der Abgrenzbarkeit der anterioren und

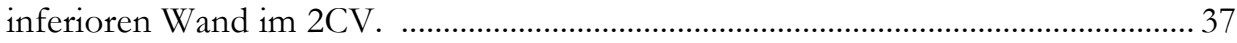

Abbildung 19: Bubble-Plots für die Bewertung des Septums in den langen Achsen. ......................38

Abbildung 20: Bubble-Plots für die Bewertung des Septums in den kurzen Achsen. ...................... 39

Abbildung 21: Bubble-Plots für die Bewertung der Seitenwand in den langen Achsen. ................. 40

Abbildung 22: Bubble-Plots für die Bewertung der Seitenwand in den kurzen Achsen. ................ 41

Abbildung 23: Bubble-Plots für die Qualitätsanalyse der Abgrenzbarkeit der Papillarmuskeln. .... 42

Abbildung 24: Bland-Altman-Plots zum Vergleich zwischen CINE- und Real-Time-SSFP-SAStapel für EDV und EF.

Abbildung 25: Bland-Altman-Plots zum Vergleich zwischen CINE- und Real-Time-SSFP4CV für EDV und EF.

Abbildung 26: Bland-Altman-Plots zum Vergleich zwischen CINE-SSFP-4CV und Echokardiographie sowie Real-Time-SSFP und Echokardiographie für EDV und EF.

Abbildung 27: Bland-Altman-Plots für den Vergleich der Mittelung über sieben Schläge mit der Mittelung über fünf Schläge und drei Schläge des Real-Time-SSFP-4CV für EDV und EF.

Abbildung 28: Spannweite der EF bei allen Patienten über sieben Schläge. 


\section{Tabellenverzeichnis}

Tabelle 1: Absolute und relative Häufigkeiten des Geschlechts der 30 Patienten. ........................... 14

Tabelle 2: Deskriptive Kenngrößen für die demographischen Variablen. ........................................ 14

Tabelle 3: Parameter für die Bildakquisition der CINE- und Real-Time-Bilder. ............................... 17

Tabelle 4: Score zur Beurteilung der Diagnosequalität. ..................................................................... 19

Tabelle 5: Kenngrößen zur Verteilung der Schlagdauer (ms) pro Patient. ....................................... 29

Tabelle 6: Qualitätsanalyse Gesamteindruck - Ergebnisse des Wilcoxon-matched-pairs-Tests. .... 30

Tabelle 7: p-Werte für Qualitätsanalyse der Abgrenzbarkeit der anterioren und inferioren Wand - Ergebnisse des Wilcoxon-matched-pairs-Tests. . .............................................. 37

Tabelle 8: p-Werte für Qualitätsanalyse der Abgrenzbarkeit des Septums - Ergebnisse des Wilcoxon-matched-pairs-Tests. ................................................................................... 38

Tabelle 9: p-Werte für Qualitätsanalyse der Abgrenzbarkeit der Seitenwand - Ergebnisse des Wilcoxon-matched-pairs-Tests.

Tabelle 10: p-Werte für Qualitätsanalyse der Sichtbarkeit und Abgrenzbarkeit der Papillarmuskeln: Ergebnisse des Wilcoxon-matched-pairs-Tests. ................................. 42

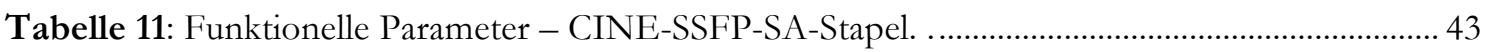

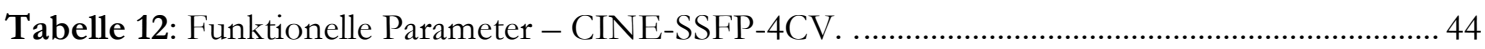

Tabelle 13: Funktionelle Parameter - Real-Time-SSFP-SA-Stapel. ..................................................... 45

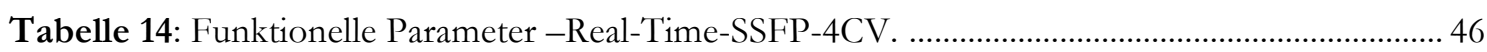

Tabelle 15: Funktionelle Parameter - Echokardiographie. ................................................................ 47

Tabelle 16: Bland-Altman-Analyse - Vergleich Real-Time-SSFP- vs. CINE-SSFP-SA-Stapel. ...... 48

Tabelle 17: Bland-Altman-Analyse: Vergleich Real-Time-SSFP- vs. CINE-SSFP-4CV . .................. 49

Tabelle 18: Bland-Altman-Analyse - CINE-SSFP-4CV vs. Echokardiographie. ............................. 50

Tabelle 19: Bland-Altman-Analyse - Vergleich der funktionellen Analyse des Real-TimeSSFP-4CV über sieben, fünf und drei Schläge. 


\section{Abkürzungsverzeichnis}

\begin{tabular}{|c|c|}
\hline $2 \mathrm{CV}$ & Zweikammerblick \\
\hline $3 \mathrm{CV}$ & Dreikammerblick \\
\hline $4 \mathrm{CV}$ & Vierkammerblick \\
\hline ED & Enddiastole \\
\hline EDV & Enddiastolisches Volumen \\
\hline EKG & Elektrokardiogramm \\
\hline ES & Endsystole \\
\hline FID & free induction decay: eine Art MRT-Signal \\
\hline HASTE & half fourier-acquired single shot turbo spin echo: MRT-Sequenz \\
\hline HF-Impuls & Hochfrequenzimpuls \\
\hline MM & Myokardiale Masse \\
\hline MRT & Magnetresonanztomographie \\
\hline NLINV & Nichtlineare Inversion \\
\hline NMR & nuclear magnetic resonance: Kernspinresonanz \\
\hline SA & short axis: kurze Achse \\
\hline SA apex & short axis apical: apikaler Kurzachsenschnitt \\
\hline SA basis & short axis basis: basaler Kurzachsenschnitt \\
\hline SA mid & short axis mid: midventrikulärer Kurzachsenschnitt \\
\hline SA-Stapel & Kurzachsenstapel \\
\hline SSFP & steady state free precession: MRT-Sequenz \\
\hline Stabw & Standardabweichung \\
\hline TE & time to echo: Echozeit \\
\hline TR & time to repeat: Repetitionszeit \\
\hline TrueFISP & true fast imaging with steady state free precession: MRT-Sequenz \\
\hline TTE & Transthorakale Echokardiographie \\
\hline$\ddot{U} G r$ & Übereinstimmungsgrenze \\
\hline UMG & Universitätsmedizin Göttingen \\
\hline Unt. Dat. & Untersuchungsdatum \\
\hline
\end{tabular}




\section{$1 \quad$ Einleitung}

\subsection{Stellenwert der MRT in der Herzbildgebung}

Für viele kardiologische Therapieentscheidungen ist eine qualitativ hochwertige Bildgebung von entscheidender Bedeutung. Die nichtinvasive kardiologische Diagnostik hat sich vom konventionellen Röntgenbild über die Echokardiographie mitsamt ihren Erweiterungen wie Stressechokardiographie und 3 D-Echokardiographie bis hin zur nuklearmedizinischen Diagnostik und Computertomographie entwickelt. In den letzten Jahrzehnten hat sich parallel dazu eine weitere Technik in der Diagnostik etabliert: die kardiale Magnetresonanztomographie (MRT).

Die MRT basiert auf der Eigenbewegung von Atomkernen (Spin) in einem konstanten magnetischen Feld, die sich bei der Aussendung von Hochfrequenzimpulsen verändert und anschließend in ihren Ausgangszustand zurückfindet, wodurch ein Resonanzsignal entsteht. Das Prinzip wurde erstmals von Bloch (1946) und etwa zeitgleich von Purcell et al. (1946) beschrieben. In der Gewebedarstellung wird hauptsächlich der Spin der Wasserstoffkerne (Protonen) betrachtet. Einzug in die medizinische Bildgebung fand die MRT durch die Erkenntnisse zur Ortskodierung von Lauterbur (1973). Mansfield und Maudsley (1977) beschrieben die wesentlichen Grundlagen der morphologischen Bildgebung. Erste Ansätze der kardialen MRT entwickelten sich in den frühen 1980er Jahren. So proklamierten beispielsweise Goldman et al. (1980) die MRT als eine vielversprechende Methode für die Herzbildgebung, während Hawkes et al. (1981) als eine der ersten Arbeitsgruppen morphologische Darstellungen vom Herzen als MRT-Bilder präsentierten. Heute ist die kardiale MRT bei verschiedensten klinischen Fragestellungen Methode der Wahl, bedingt durch viele Vorteile, die das Verfahren mit sich bringt:

Die zeitliche und räumliche Auflösung ist besser als bei den nuklearmedizinischen Methoden und es entsteht keine Strahlenbelastung (Saeed et al. 2015). Ein Vorteil gegenüber der Echokardiographie ist die Möglichkeit der beliebigen Schichtführung. Die in der Echokardiographie typischen Limitationen durch das akustische Fenster, das von den körperlichen Voraussetzungen des Patienten beeinflusst wird (Buck et al. 2009; Omoto 1982; Siadecki et al. 2015), treten in der MRT nicht auf. Echokardiographie und MRT ermöglichen die nichtinvasive dynamische Darstellung der Herzbewegung, wobei die MRT eine noch sicherere Definition der Endokardgrenzen erlaubt (Saeed et al. 2015).

Für die Analyse der Funktion des linken und rechten Ventrikels dient die kardiale MRT heute in zahlreichen Studien als Referenzstandard (Dewey et al. 2006; Zhou et al. 2015). Weiterhin ist die Methode zur Untersuchung von Vitalität, Funktionsfähigkeit, Ödemausbildung und Fibrosierung sowie Perfusion des Myokards (Saeed et al. 2015) 
geeignet, wodurch sie hohe Bedeutung in der Diagnostik der koronaren Herzkrankheit, der verschiedenen Kardiomyopathien und der Myokarditis (Friedrich und Marcotte 2013) erhält. Durch diese Untersuchungen kann z.B. die Frage nach der Indikation einer Herzkatheterdiagnostik geklärt werden, was zur Vermeidung unnötiger Risiken führt und erhebliche Kosten einspart (Petrov et al. 2015). Bei Patienten mit angeborenen Herzfehlern lassen sich die anatomischen Lageverhältnisse und Blutflüsse genau beurteilen, was zur OPPlanung und Verlaufskontrolle wichtig ist.

Die MRT wird also heute in der Diagnostik vieler wichtiger Herzerkrankungen angewandt. Die Standardsequenzen beruhen - wie in Kapitel 1.3 näher beschrieben - auf einer EKGabhängigen Interpolation der Bilddaten über mehrere Herzschläge. Daraus ergeben sich einige Limitationen. Das EKG-Signal kann durch das Magnetfeld des Gerätes gestört werden. Die Sequenzen erfordern für eine gute Bildqualität Atemruhelage, sodass bei Patienten, die die Luft nicht lange anhalten können, die optimale Qualität oft nicht erreicht werden kann. Insbesondere bei Herzrhythmusstörungen kann die Bildqualität eingeschränkt sein, da die Herzbewegung sehr unregelmäßig ist. Mit einem Verfahren, das die Herzbewegung EKG-unabhängig, in Echtzeit und mit hoher zeitlicher Auflösung abbildet, hofft man, insbesondere bei diesen Patienten hochwertigere Bilder zu erhalten, was die Nachverarbeitung und Beurteilung erleichtern würde.

\subsection{Typische Sequenzen in der kardialen MRT}

In der Herzbildgebung werden häufig Gradientenechosequenzen verwendet. Sie beginnen mit einem Hochfrequenzimpuls (HF-Impuls). Dieser lenkt die Protonenbewegung aus, die vorher entlang des homogenen Magnetfelds des Tomographen ausgerichtet ist (Longitudinalmagnetisierung), wobei sich eine Magnetisierungskomponente quer zum Magnetfeld entwickelt (Transversalmagnetisierung). Dabei entsteht ein neuer Hauptvektor der Magnetisierung. Der Winkel zwischen diesem und der Richtung des homogenen Magnetfelds wird als Flipwinkel bezeichnet. Die Protonenbewegung findet schnell in den Ausgangszustand zurück (Relaxation), wobei ein Signal entsteht. Anschließend werden Magnetfeldgradienten geschaltet, die die Transversalmagnetisierung wiederholt zerstören und wieder aufbauen, sodass Echosignale entstehen. Die Zeit bis zu einem Echosignal ist die Echozeit (time to echo - TE). Diese Serie aus HF-Impulsen und Gradienten wird nach jeder Repetitionszeit (time to repeat - TR) wiederholt. Alle Signale werden vom Rückgang der Transversalmagnetisierung (Zeitkonstante T1) und vom Wiederherstellen der Longitudinalmagnetisierung (Zeitkostante T2) beeinflusst, die voneinander unabhängig sind. Signale, die eher vom Wiederherstellen der Longitudinalmagnetisierung beeinflusst werden, sind „T1-gewichtete“ Signale und solche, die eher vom Rückgang der Transversalmagnetisierung beeinflusst werden, sind „T2-gewichtet“. „T2*-gewichtete“ Signale hängen vom nicht durch Gradientenschaltung beeinflussten Rückgang der Transversalmagnetisierung ab. T1, T2 und T2* unterscheiden sich je nach Gewebetyp. 
Ob Signale T1-, T2- oder T2*-gewichtet sind, hängt von der Gradientenschaltung ab. Zwei typische Gradientenechosequenzen, die für das Verständnis dieser Arbeit wichtig sind, werden im Folgenden vorgestellt.

\subsubsection{FLASH-Sequenz}

Der Fast Low Angle Shot (FLASH) wurde erstmals beschrieben von Haase et al. (1986). Es handelt sich um eine Gradientenecho-Sequenz, die mit einem HF-Impuls mit kleinem Flipwinkel, z.B. $15^{\circ}$, beginnt. Durch den kleinen Flipwinkel trifft ein neuer HF-Impuls nach schneller Gradientenschaltung auf eine ausreichende Longitudinalmagnetisierung, sodass TR sehr kurz gewählt werden kann. Nach einigen TR stellt sich ein Gleichgewicht aus Anregung und Relaxation bis hin zum steady-state ein. Die verbleibende Transversalmagnetisierung wird durch Schalten eines Spoilergradienten am Ende jeder TR zerstört (Rauschenberg 2008). FLASH-Sequenzen sind T1- oder T2*-gewichtet. Für die Bildgebung von Herz und Blutgefäßen ist die FLASH-Sequenz gut geeignet, da durch Inflow-Effekte bewegte Flüssigkeiten hell erscheinen, statische Flüssigkeiten hingegen dunkel (Zhang 2009).

\subsubsection{Balanced-steady-state-free-precession-(bSSFP)-Sequenz}

Die formalen Grundlagen der steady-state-free-precession wurden bereits 1958 beschrieben (Carr 1958). Dennoch ist die balanced-steady-state-free-precession (bSSFP) erst seit Ende der 90er Jahre in der klinischen Anwendung etabliert (Scheffler und Lehnhardt 2003). Bei der bSSFP gibt es nicht wie bei der FLASH-Sequenz einen Spoilergradienten, allerdings existiert zu jedem Gradienten ein Gegengradient gleicher Stärke. Dadurch kommt es über eine TR hinweg nicht zur Dephasierung; die Transversalmagnetisierung bleibt erhalten und überlagert sich mit der Transversalmagnetisierung des nächsten HFImpulses (Ridgway 2010). Durch die kurze TR und die konstante Quermagnetisierung stellt sich schnell ein steady-state ein. Sowohl Longitudinal- als auch Transversalmagnetisierung beeinflussen also das Signal, welches vom Verhältnis von T2 und T1 abhängig ist. Flüssigkeiten und Fettgewebe werden in der bSSFP hell dargestellt. Zwar haben die beiden Substanzen sehr unterschiedliche T2- und T1-Werte, der Quotient aus beiden ist jedoch ähnlich. Flüssigkeiten, die in die betrachtete Schicht hineinfließen haben den steady-state noch nicht erreicht und geben dadurch ein stärkeres Signal und das Blut wird noch heller dargestellt (Scheffler und Lehnhardt 2003). Deutlich weniger signalintensiv ist hingegen die Muskulatur und damit das Myokard. Wegen des guten Kontrasts zwischen Blut und Myokard werden bSSFP-Sequenzen in der Herz-MRT bevorzugt eingesetzt.

\subsection{CINE-SSFP: Standard in der Herzbildgebung}

MR-Signale enthalten Rohdaten zu Ort und Kontrast, die für die Bildberechnung wichtig sind. Für ein Bild sind Informationen aus mehreren MR-Signalen erforderlich, die zunächst 
in eine virtuelle Matrix, den sogenannten k-Raum, eingetragen werden. Dessen vollständige Füllung nimmt einige Zeit (Sekunden) in Anspruch. Das Herz befindet sich jedoch ständig in Bewegung. Um eine Momentaufnahme des Herzens zu gewinnen, darf die Bildakquisition also nur wenige ms dauern. Dieses Problem wird umgangen, indem die Informationen für ein einziges Bild über mehrere Herzschläge interpoliert werden. Es entsteht eine Bildserie, die alle Herzphasen zeigt und wie ein kleiner Kinofilm abgespielt werden kann, eine sogenannte CINE-Sequenz. Um die Rohdaten der richtigen Herzphase zuordnen zu können, muss während der Akquisition ein EKG aufgezeichnet werden. Die MRT-Betriebssoftware kann dann die Pulssequenz mit dem EKG synchronisieren (Ridgway 2010). So können Datenakquisition und Herzschlag aufeinander abgestimmt werden. Hierbei gibt es zwei verschiedene Vorgehensweisen:

Beim prospektiven Triggern werden für jede EKG-definierte Herzphase pro Schlag einige Rohdatenzeilen aufgenommen. Die Akquisition beginnt kurz nach der R-Welle und endet vor der nächsten R-Welle. Dies verlangt die Berechnung eines Standard-RR-Intervalls, damit die Pulssequenz rechtzeitig gestoppt wird. Zu jeder definierten Herzphase gibt es einen k-Raum. Das Vorgehen wird so lange wiederholt, bis alle k-Räume gefüllt sind (Abbildung 1). Das prospektive Triggern hat den Nachteil, dass während des QRSKomplexes keine Daten akquiriert werden (Ridgway 2010).

Beim retrospektiven Gating (Lenz et al. 1989) werden konsekutiv Rohdaten mit kurzer TR akquiriert. Jeder Rohdatenzeile wird das EKG-Signal zugeordnet. Die Akquisition läuft so lange, bis ausreichend Rohdaten vorhanden sind, um alle Bilder eines vollständigen Herzzyklus zu berechnen. Anschließend werden die Rohdaten anhand des EKG-Signals sortiert, wie unten in Abbildung 1 dargestellt (Ridgway 2010). Die CINE mit retrospektivem Gating ermöglicht die Abbildung aller Herzphasen, was besonders für die Analyse der Herzfunktion von Bedeutung ist.

CINE-Aufnahmen gelingen meist nur in Atemruhelage. Je nach Bildanzahl und TR werden meist zwischen zehn und 20 Herzschläge benötigt (Bluemke et al. 1997; Kellman et al. 2009), wofür ca. 15 Sekunden lang die Luft angehalten werden muss. Um den linken Ventrikel komplett von apikal bis basal abzudecken, sind acht bis zwölf Schichten (Schichtdicke ca. $8 \mathrm{~mm}$ ) in Atemruhelage erforderlich (Moon et al. 2002), was für ältere Menschen oder Patienten mit Luftnot oft nur schwer möglich ist. Messungen unter freier Atmung und kürzere Messzeiten würden die Untersuchung erleichtern. Weiterhin können EKG-abhängige Aufnahmen durch den magnetohydrodynamischen Effekt an Bildqualität verlieren: Beim Blutfluss durch ein magnetisches Feld kommt es zu Spannungsschwankungen, die das EKG-Signal stören können (Nijm et al. 2008). Dennoch gilt die auch in dieser Studie verwendete Kombination aus CINE und bSSFP-Sequenz als Methode der Wahl für die Funktionsanalyse des Herzens (Kramer et al. 2013). Sie wird im Folgenden als CINE-SSFP bezeichnet. 


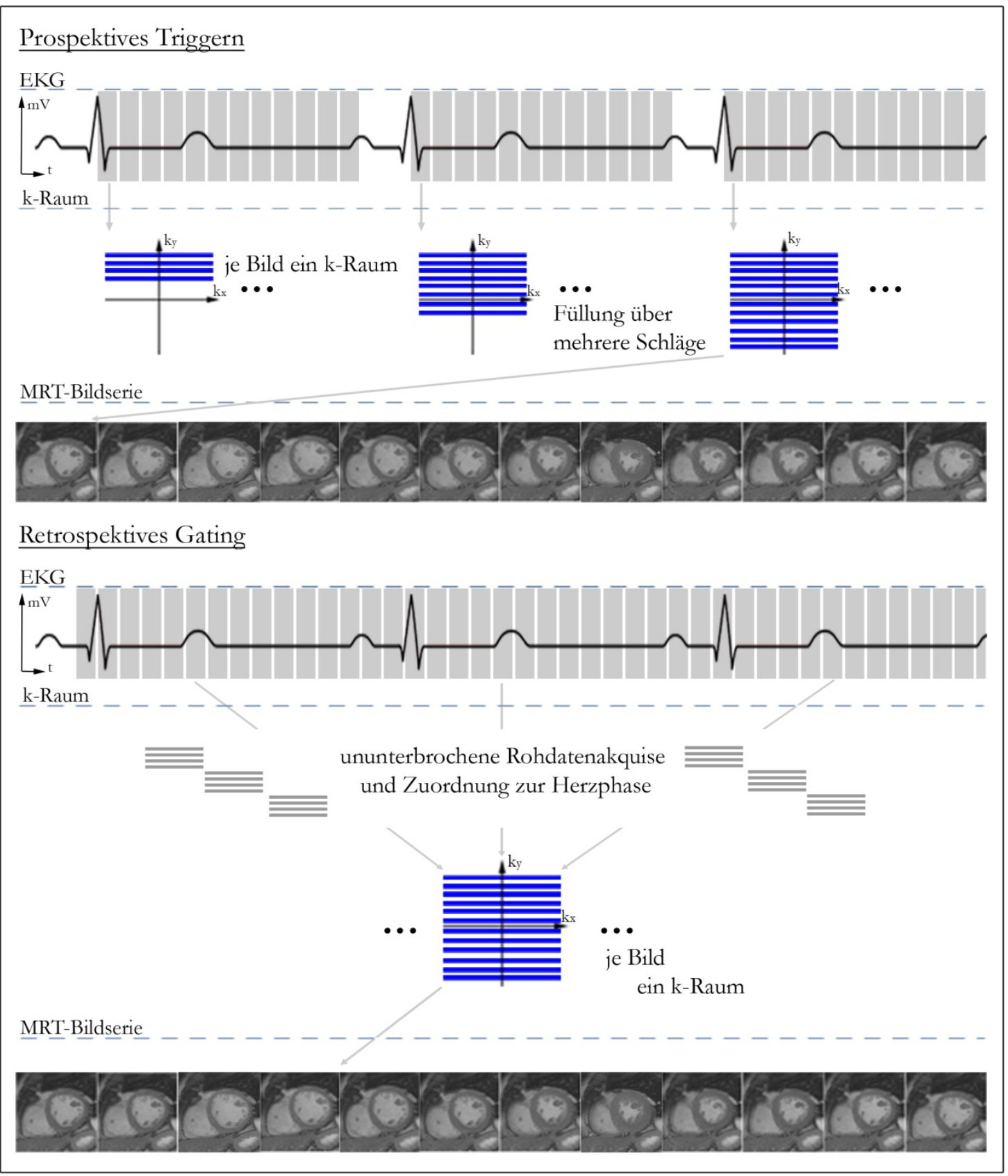

Abbildung 1: Prinzip der CINE-SSPP - prospektives Triggern und retrospektives Gating. Beim prospektiven Triggern (oben) wird die Datenakquisition mit der R-Welle gestartet und endet rechtzeitig vor der nächsten R-Welle. Jeder Herzphase wird ein k-Raum zugeordnet, der über mehrere Herzschläge hinweg mit Rohdaten aufgefüllt wird, bis ausreichend Informationen vorhanden sind, um ein interpoliertes Bild zu berechnen. Beim retrospektiven Gating (unten) werden die Rohdaten zunächst konsekutiv und ohne Unterbrechung akquiriert und dann anhand eines EKG-Stempels der richtigen Herzphase und dem zugehörigen k-Raum zugeordnet. Die Zuordnung erfolgt also unabhängig von Start und Stopp der Messung und der gesamte Herzzyklus kann abgebildet werden. Zur Veranschaulichung sind hier nur drei Schläge dargestellt, tatsächlich erfolgt die Interpolation über etwa zehn bis 20 Schläge. Repräsentativ ist nur ein k-Raum dargestellt. Die tatsächliche Anzahl an k-Räumen entspricht der Anzahl der Bilder pro Serie. 


\subsection{Echtzeit-MRT: Real-Time-SSFP}

Das Ziel der Echtzeit-MRT ist es, Bilder mit sehr hoher zeitlicher Auflösung und gleichzeitig guter Ortsauflösung zu erhalten. In der Herz-MRT liegt der Vorteil eines Echtzeitverfahrens darin, dass Bilder vom Herzschlag konsekutiv gewonnen werden können und keine Interpolation über mehrere Herzschläge mehr nötig ist. Erste Ansätze der Echtzeit-MRT am Herzen wurden bereits 1987 entwickelt (Chapman et al. 1987; Rzedzian und Pykett 1987). Seitdem entstanden immer wieder neue Verfahren (Bauer et al. 2013; Muthurangu et al. 2008; Spuentrup et al. 2003), die sich im klinischen Alltag jedoch noch nicht durchsetzen konnten. Das große Problem ist, dass eine Optimierung der zeitlichen Auflösung häufig auf Kosten der räumlichen Auflösung geht (Contijoch et al. 2015). Die Arbeitsgruppe um Prof. Dr. Jens Frahm am Max-Planck-Institut für biophysikalische Chemie („Biomedizinische NMR Forschungs GmbH“) in Göttingen hat auf Basis der FLASH-Sequenz ein Echtzeitverfahren entwickelt, das eine zeitliche Auflösung von $40 \mathrm{~ms}$ und höher bei einer Ortsauflösung von unter $2 \cdot 2 \mathrm{~mm}^{2}$ aufweist. Diese Parameter sind mit etablierten MRT-Verfahren vergleichbar (Zhang et al. 2010). Dies wird durch eine Unterabtastung der Rohdaten erreicht, d.h. der k-Raum wird nicht vollständig Zeile für Zeile aufgefüllt, sondern in Form einiger radialer Speichen. Die Bildberechnung erfolgt mit einem Rekonstruktionsverfahren, das als ,iterativ regularisierte nicht-lineare Inversion“ (NLINV) bezeichnet wird (Uecker et al. 2010a). Für die NLINVRekonstruktion ist ein Computersystem mit mehreren Grafikkarten erforderlich, das mit den Rechnern der MRT-Workstation verbunden ist. Da die Rekonstruktion unmittelbar auf die Datenakquisition folgt, können die Bilder schon nach wenigen Sekunden - teilweise noch während der laufenden Messung - auf dem Bildschirm der MRT-Workstation betrachtet werden. Die Datenakquisition kann EKG-unabhängig und unter freier Atmung erfolgen. Das Prinzip der Real-Time-Sequenz ist in Abbildung 2 schematisch dargestellt.

Verschiedene Studien haben bereits gezeigt, dass das beschriebene Verfahren für die klinische Anwendung, z.B. bei Patienten mit Schluckstörungen oder gastrointestinaler Refluxkrankheit, geeignet ist (Olthoff et al. 2014; Zhang et al. 2015). Studien zu Flussmessungen zeigten die Eignung auf dem Gebiet der kardiovaskulären Bildgebung (Fasshauer et al. 2014; Joseph et al. 2014; Kowallick et al. 2014). Dieses neue Echtzeitverfahren, das zunächst basierend auf der FLASH-Sequenz entstand, wurde dann auch auf Basis einer bSSFP-Sequenz und bei einer Magnetfeldstärke von 1,5 Tesla weiterentwickelt. Voit et al. (2013) verglichen das Verfahren mit der herkömmlichen CINE-SSFP hinsichtlich Bildqualität und Analyse der linksventrikulären Funktion. Dabei arbeiteten sie mit einer zeitlichen Auflösung von 40,67 ms und einer räumlichen Auflösung von $1,8 \cdot 1,8 \mathrm{~mm}^{2}$. Unter den 27 untersuchten Personen waren sechs gesunde Probanden sowie 21 Patienten mit typischen kardialen Erkrankungen, auch einige mit Wandbewegungsstörungen und Arrhythmien. Die technischen Grundlagen von Voit et al. (2013) sind weitgehend auch Grundlage dieser Studie. 


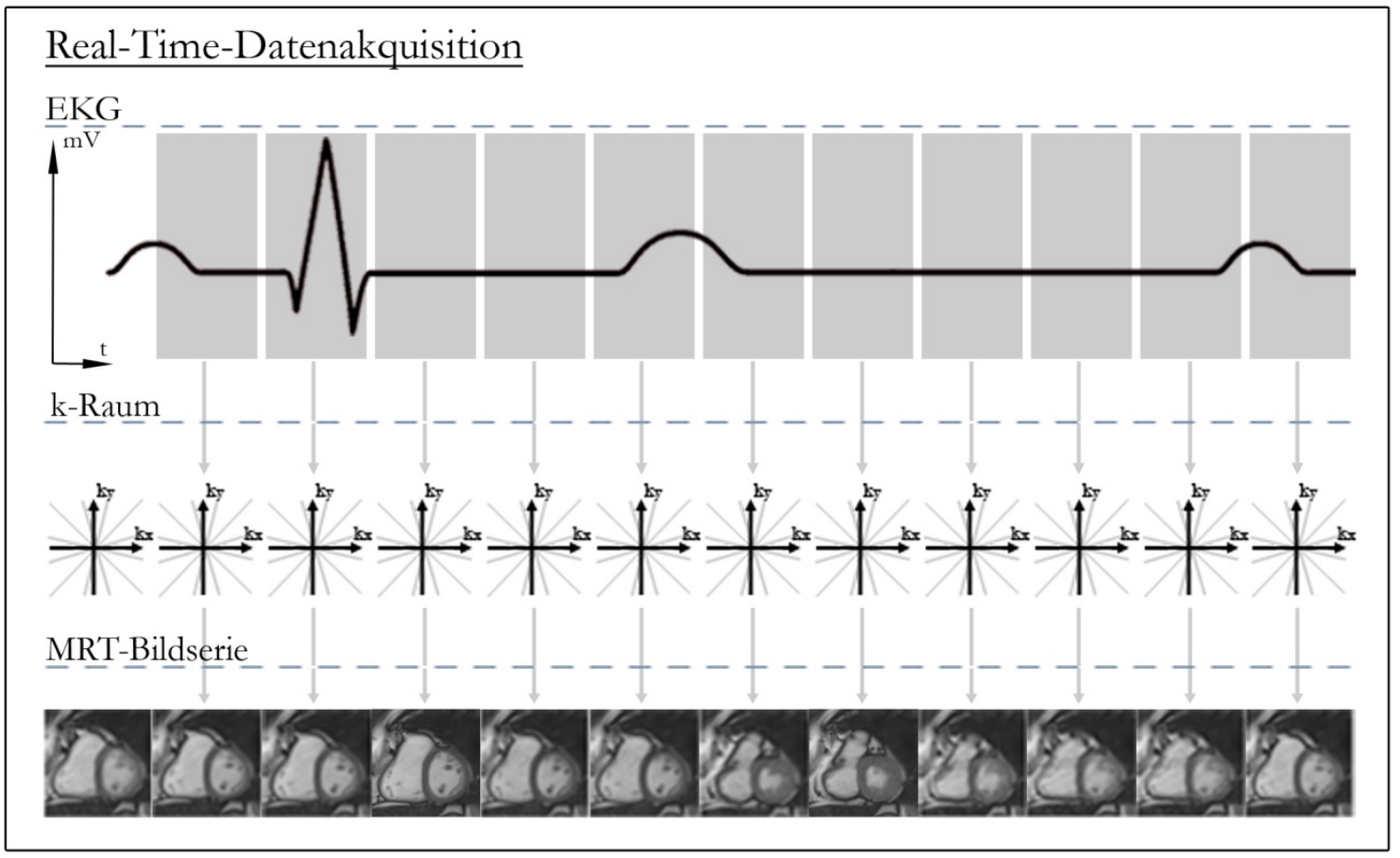

Abbildung 2: Graphische Darstellung des Prinzips der Real-Time-Datenakquisition. Durch die radiale k-Raum-Füllung und anschließende Berechnung der fehlenden Daten via NLINV können alle für ein Bild nötigen Daten in wenigen ms gewonnen werden. Dadurch ist eine Bildgebung in Echtzeit, ohne Interpolation und EKG-unabhängig möglich. Die Grafik dient der Veranschaulichung und berücksichtigt nicht die tatsächliche zeitliche Auflösung.

\subsection{Kardiale MRT bei Patienten mit Arrhythmien}

Wie in Kapitel 1.3 beschrieben, kommen prospektives Triggern und retrospektives Gating bei Patienten mit stärkeren Arrhythmien an ihre Grenzen. Die CINE-SSFP beinhaltet eine Arrhythmie-Korrektur. Schläge, deren Länge stark vom mittleren RR-Intervall abweicht, werden bei der Bildberechnung außenvorgelassen (Xue et al. 2013) - eine gute Lösung für Patienten mit gelegentlichen Extrasystolen oder gering schwankenden RR-Intervallen. Bei Patienten mit durchgehend unregelmäßigen RR-Intervallen - wie beispielsweise bei Vorhofflimmern (VHF) - genügt dieses Vorgehen oft nicht. Es kommt dann zu deutlichen Artefakten und eine klare Konturabgrenzung ist weder visuell noch mit einer automatischen Segmentationssoftware möglich. Ein Auslassen arrhythmischer Schläge mit meist schlechterer Ejektionsfraktion (EF) führt des Weiteren zur Überschätzung der Herzfunktion. Bereits Ridgway (2010) empfiehlt daher die Anwendung von Echtzeitverfahren mit EKG-unabhängiger Datenakquisition. Gleichzeitig stellt er aber auch fest, dass dies auf Kosten der räumlichen und zeitlichen Auflösung gehe. Die hier verwendete, neue Real-Time-SSFP verfügt - wie in Kapitel 1.4 beschrieben - über eine hohe räumliche und zeitliche Auflösung. Die Qualität des einzelnen Bildes wäre damit nicht mehr vom Herzrhythmus des Patienten abhängig und für die Konturabgrenzung wäre der Rhythmus weniger stark von Bedeutung. Abbildung 3 zeigt Bildbeispiele für die 
CINE-SSFP im Sinusrhythmus und bei Arrhythmien sowie für die Real-Time-SSFP bei Arrhythmien.
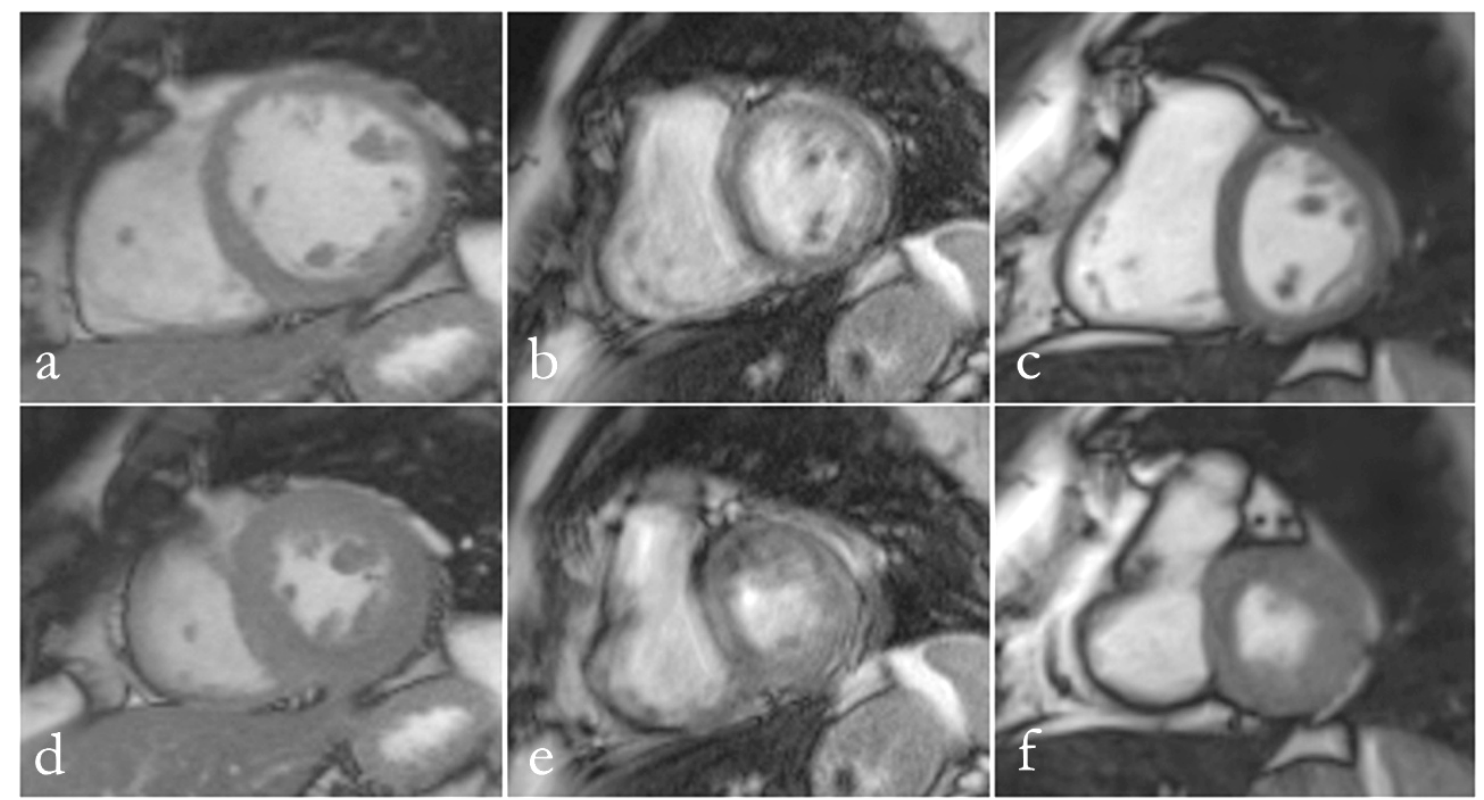

Abbildung 3: Bildbeispiele.

CINE-SSFP-Kurzachsenschicht im Sinusrhythmus $(\mathbf{a}=$ Enddiastole $/ E D, \mathbf{d}=$ Endsystole $/ \mathrm{ES})$, CINE-(b = ED, $\mathbf{e}=\mathrm{ES})$ und Real-Time-SSFP-Kurzachsenschicht $(\mathbf{c}=\mathrm{ED}, \mathbf{f}=\mathrm{ES})$ bei VHF. CINE- und Real-Time-SSFP bei VHF sind vom gleichen Patienten. Die eingeschränkte Qualität der CINE-SSFP unter Arrhythmie fällt deutlich ins Auge.

\subsection{Definition des Krankheitsbildes Vorhofflimmern (VHF)}

Um zuverlässig MRT-Datensätze bei unterschiedlichen RR-Intervallen zu evaluieren, wurden Patienten mit VHF untersucht. VHF ist mit einer Prävalenz von ein bis zwei Prozent die häufigste supraventrikuläre Herzrhythmusstörung (Go et al. 2001; Stewart et al. 2001). Die Überleitung auf die Ventrikel ist von der Funktion des AV-Knotens abhängig. Es resultiert eine unregelmäßige Überleitung, wobei die RR-Intervalle sehr unterschiedlich schwanken können und damit das Ausmaß der Arrhythmie für die Ventrikelaktion bestimmen. Hinsichtlich der Herzfrequenz unterscheidet man tachykarde (Herzfrequenz $(\mathrm{HF})>100$ Schläge pro Minute), normofrequente (HF 60 bis 100 Schläge pro Minute) und bradykarde (HF < 60 Schläge pro Minute) Formen des resultierenden Ventrikelrhythmus. 


\subsection{Schnittführung in der kardialen MRT}

In der kardialen MRT-Bildgebung soll die Morphologie der Herzkammern vollständig erfasst werden. Ein Vorteil dieser Methode ist die Möglichkeit der beliebigen Schichtführung. In einem standardisierten Untersuchungsprotokoll zur morphologischen Darstellung (Kramer et al. 2013) sind folgende Schnittebenen erhalten:

- Vierkammerblick (4CV) - dieser Langachsenschnitt steht senkrecht auf der Herzspitze, schneidet die Mitral- und Trikuspidalklappenebene mittig und zeigt beide Ventrikel und beide Vorhöfe.

- Zweikammerblick (2CV) - schneidet den linken Ventrikel orthogonal zum 4CV und halbiert ebenfalls die Mitralklappenebene; linker Ventrikel und linker Vorhof sind abgebildet.

- Dreikammerblick (3CV) - schneidet ebenfalls den Apex und die Mitralklappenebene und gleichzeitig mittig die Aortenklappe; linker Ventrikel, linker Vorhof und linksventrikulärer Ausflusstrakt sind zu sehen.

- Kurzachsenstapel (SA-Stapel) - mehrere, orthogonal zum 4CV und parallel zur Klappenebene verlaufende Schnitte, zeigt die beiden Ventrikel und bildet das gesamte Ventrikelmyokard ab. Alle SA-Schichten zusammen ergeben als Stapel eine dreidimensionale Repräsentation der Ventrikel.

Die beschriebenen Schnittebenen sind in Abbildung 4 dargestellt. 
$\underline{\text { Schnittführung in der kardialen MRT }}$

$2 \mathrm{CV}=$ Zweikammerblick

$3 \mathrm{CV}=$ Dreikammerblick

$4 \mathrm{CV}=$ Vierkammerblick

Ao $=$ Aorta

$\mathrm{LA}=$ linkes Atrium

$\mathrm{LV}=$ linker Ventrikel

$\mathrm{RA}=$ rechtes Atrium

$\mathrm{RV}=$ rechter Ventrikel

$\mathrm{SA}=$ kurze Achse
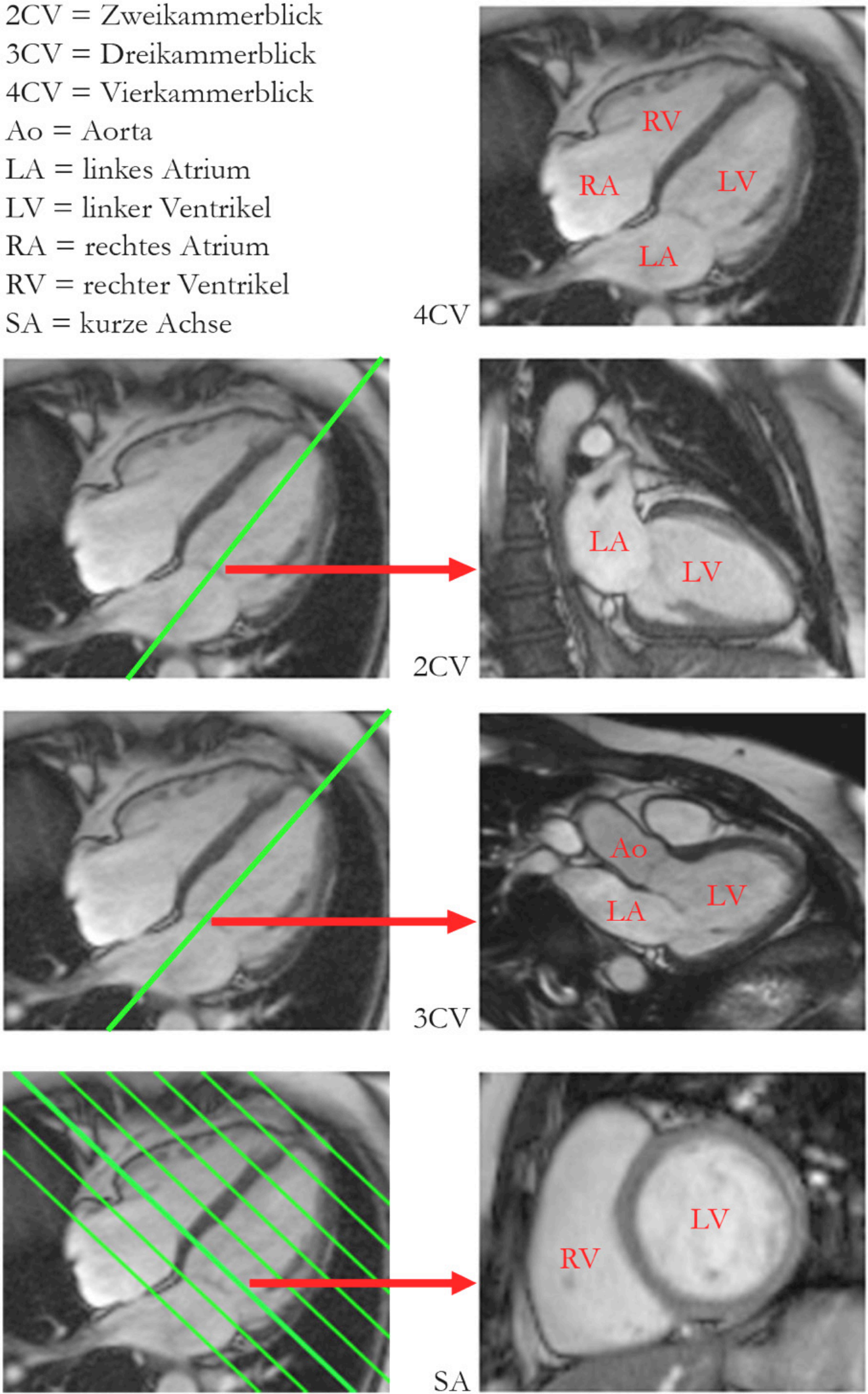

Abbildung 4: Schnittführung in der kardialen MRT.

Die Abbildung zeigt den 4CV sowie die Lage von 2CV, 3CV und SA relativ zum 4CV; die Beschriftung markiert die auf den Schnittebenen dargestellten Kammern und Vorhöfe. 


\subsection{Diagnostische Verwertung von Kardio-MRT-Bildern}

Die Auswertung der akquirierten MRT-Daten des Herzens besteht zum einen in der Beurteilung von Morphologie und Funktion, zum anderen in der Nachverarbeitung und Segmentation zur Erhebung quantitativer funktioneller Daten. Letzteres geschieht mithilfe einer softwarebasierten Konturdetektion. Die klinische Beurteilung der erhobenen Befunde erfolgt dann durch einen erfahrenen Untersucher.

Wie bei allen radiologischen Aufnahmen ist es wichtig, dass Kardio-MRT-Bilder eine gute diagnostische Qualität haben, damit die Morphologie sicher beurteilt werden kann. In der Herzbildgebung sollten z.B. Wandbewegungsstörungen oder Infarktzonen gut erkannt werden können. Um herauszufinden, ob ein radiologisches Verfahren für den klinischen Alltag geeignet ist, muss die diagnostische Qualität gewonnener Bilder evaluiert werden. Hierfür wird häufig eine Ordinalskala nach Likert (1932) verwendet (Bassett et al. 2014; Kino et al. 2009; Leschka et al. 2007), anhand derer erfahrene Untersucher die Bildqualität bewerten. Voit et al. (2013) verwendeten ebenfalls eine Likert-Skala, um die Qualität der Real-Time-SSFP-Bilder mit der der CINE-SSFP-Bilder zu vergleichen. Die gleiche Skala wurde auch in der hier vorliegenden Studie verwendet, in der zwei erfahrene Untersucher die Bilder bewerteten. Zusätzlich zum Gesamteindruck erfolgte noch eine gesonderte Bewertung wichtiger anatomischer Strukturen (siehe Kapitel 2.4).

Die softwarebasierte Nachanalyse von Kardio-MRT-Bildern ist insbesondere für die Beurteilung der ventrikulären Funktion von Bedeutung (Schulz-Menger et al. 2013). Es gibt verschiedene Softwarelösungen zur Nachanalyse von Kardio-MRT-Bildern, z.B. Mass (van der Geest et al. 1994) oder Argus (O’Donnell et al. 2006), die dem gleichen Grundprinzip folgen: Das Volumen des Blutpools und das des umgebenden Myokards müssen bestimmt werden, um daraus die myokardiale Masse, die Füllungsvolumina und schließlich die Auswurfleistung des Herzens zu errechnen. Dafür muss eine Kontur um das Endokard sowie um das Epikard gelegt werden. Einige Softwareprodukte ermöglichen je nach Schnittebene zusätzlich die Segmentation der Papillarmuskeln. Auf Grundlage der durch diese Konturen umgrenzten Flächen vermag die Software das gesuchte Volumen näherungsweise $\mathrm{zu}$ bestimmen - beispielsweise mit einer biplanen oder einer Scheibchensummationsmethode (Attili et al. 2010). Für die Ventrikelvolumetrie ist die Segmentation in Enddiastole (ED) und Endsystole (ES) entscheidend. Wird der gesamte Herzzyklus segmentiert, kann die Füllungsdynamik gut untersucht werden. Das Erstellen der Konturen kann manuell, halbautomatisch oder vollautomatisch erfolgen. Bei der manuellen Segmentation muss der Anwender in jedes zu segmentierende Bild die Konturen einzeichnen (Abbildung 5). Halbautomatische Segmentationsprozesse basieren auf einer automatischen Segmentation, an die sich eine manuelle Nachkorrektur der Konturen anschließt, während das Programm bei der vollautomatischen Segmentation alle Konturen vollständig allein einzeichnet. Meist muss der Anwender vorher in einem Bild der Serie die Konturen einzeichnen, damit das Programm sie dann auf den Rest der Bildserie propagiert. 
Während jede automatische Segmentation rein bildbasiert erfolgt, kann in die manuelle Segmentation das Wissen über Anatomie und Biomechanik des Herzens mit einfließen, was insbesondere für Patienten mit Arrhythmien, Wandbewegungsstörungen oder reduzierter ventrikulärer Funktion wichtig ist. Weiterhin ist auf einigen Bildern die Myokardgrenze so verwaschen, dass eine Segmentation auf Basis der Bildinformation allein nicht adäquat gelingt (Petitjean und Dacher 2011). In dieser Studie erfolgte die Segmentation manuell mit QMass (Medis, Leiden, Niederlande) im Konsens mit zwei erfahrenen Untersuchern, um die klinische Genauigkeit der Konturen bei der besonderen Patientengruppe mit dem Krankheitsbild VHF zu sichern. Aufgrund des hohen Zeitaufwandes der manuellen Segmentation wäre für den klinischen Alltag die automatische Segmentation vorzuziehen; dies ist für die Real-Time-SSFP jedoch mit einigen Herausforderungen verbunden: Durch die hohe zeitliche Auflösung entsteht eine sehr große Menge an Bildern. Wird ein dreidimensionaler Stapel generiert, müssen mehrere tausend Bilder gleichzeitig geladen werden. Die automatsche Konturerkennung erfordert eine sehr hohe Rechenleistung. Außerdem unterscheiden sich die Real-Time-Aufnahmen hinsichtlich des Kontrastes von den herkömmlichen CINE-Bildern, was eine automatische Segmentation zusätzlich erschwert.
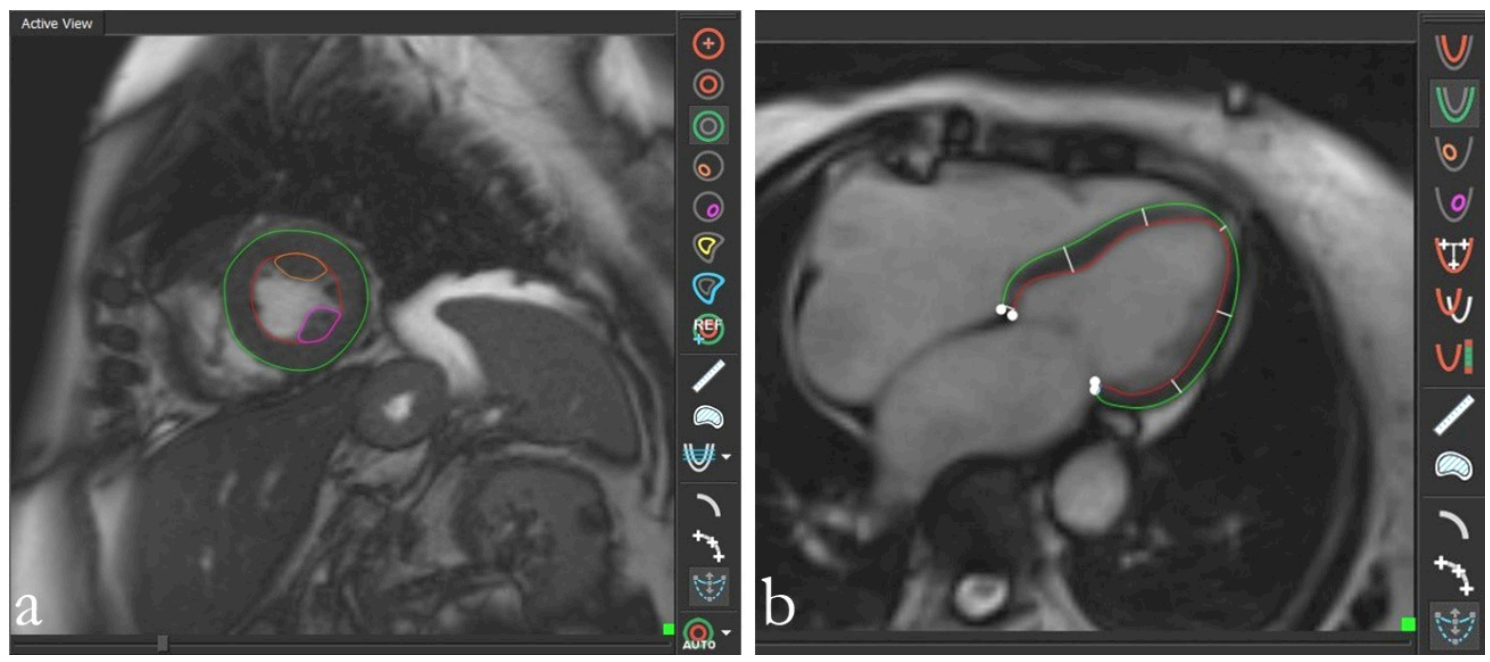

Abbildung 5: Segmentation mit QMass am Beispiel eines Bildes aus dem CINE-SSFP-SA-Stapel (a) und eines Real-Time-SSFP-4CV (b).

Grün: Epikard, rot: Endokard, orange und pink: Papillarmuskeln. Rechts im Bild sind die Werkzeuge zur automatischen und manuellen Segmentation zu sehen.

\subsection{Vergleichsmethode: Transthorakale Echokardiographie}

Neben einem Vergleich der mit CINE- und Real-Time-SSFP erhobenen Parameter erfolgte ergänzend ein Vergleich mit aus der Echokardiographie erhobenen Werten. Die Echokardiographie ist sehr weit verbreitet. Sie gehört finanziell zu den günstigsten Methoden (Picano 2005), ist schnell verfügbar und unkompliziert anzuwenden. Da mit Schallwellen gearbeitet wird, gibt es keine Strahlenbelastung und auch sonst keine 
besonderen Risiken. Bereits Anfang der 1950er Jahre war die Methode Gegenstand der Forschung und wurde wenige Jahre später im klinischen Alltag etabliert (Edler und Lindstrom 2004). Es handelt sich also um ein sehr ausgereiftes Verfahren. Dennoch gibt es einige Limitationen: Es ist nur schwer automatisierbar und daher immer von Erfahrung und Geschick des Untersuchers abhängig. Dadurch ergibt sich eine eingeschränkte Reproduzierbarkeit, was insbesondere bei der Verwendung in klinischen Studien zu beachten ist (Hoffmann et al. 2005). Aufgrund von Atemproblemen oder körperlichen Bedingungen des Patienten, beispielsweise bei Adipositas, lassen sich in einigen Fällen nicht alle Schallfenster einstellen, sodass nur ein Teil der benötigten Bilder gewonnen werden kann (Buck et al. 2009; Siadecki et al. 2015). Der Vergleich mit der Echokardiographie diente der Plausibilitätsprüfung der erhobenen Werte, da bei Arrhythmikern die CINESSFP wegen der beschriebenen Limitationen nicht als Referenzstandard verwendet werden kann. Aufgrund der beschriebenen Limitationen sind allerdings auch die Ergebnisse der Echokardiographie nicht als Goldstandard zu betrachten.

\subsection{Ziele und Fragestellung}

Ziel dieser Arbeit war es, die Eignung des neuen Echtzeitverfahrens zur Herzdiagnostik, im Speziellen zur Analyse der linksventrikulären Funktion, bei Patienten mit Arrhythmien zu überprüfen und mit den herkömmlichen MRT Sequenzen zu vergleichen. Als Modell diente das Krankheitsbild VHF. Im Einzelnen ergaben sich folgende Fragestellungen:

1. Wie verhält sich die diagnostische Qualität der Real-Time-SSFP-Aufnahmen im Vergleich mit den CINE-SSFP-Aufnahmen des gleichen Patienten?

2. Welche Werte für die funktionellen Parameter ergeben sich bei Auswertung der Real-Time-SSFP-Aufnahmen mit einer Segmentationssoftware im Vergleich mit der Auswertung der CINE-SSFP?

3. Wie verhalten sich die durch Auswertung der Real-Time- und CINE-SSFP erhobenen Werte für die funktionellen Parameter im Vergleich mit der Echokardiographie, die derzeit bei Arrhythmiepatienten zur Funktionsdiagnostik angewandt wird?

4. Welche Veränderungen der funktionellen Parameter ergeben sich durch eine Reduzierung der Anzahl der analysierten Herzschläge in der Real-Time-SSFP?

5. Ermöglicht die Real-Time-SSFP eine Schlag-zu-Schlag-Analyse und damit die Beurteilung funktioneller Schwankungen? 


\section{Material und Methoden}

\subsection{Patientenkollektiv}

30 Patienten der Universitätsmedizin Göttingen (UMG, 18 ambulant und zwölf stationär) überwiegend Patienten des Herzzentrums - wurden prospektiv in die Studie eingeschlossen. Die wesentliche Voraussetzung war ein am Untersuchungstag im EKG dokumentiertes VHF, da sich die Patienten während der Untersuchung im VHF befinden sollten. Ausschlusskriterien waren Sinusrhythmus oder ein anderer Rhythmus am Untersuchungstag und - wie für eine MRT-Untersuchung üblich - Klaustrophobie, ein implantierter Herzschrittmacher, eine künstliche Herzklappe oder andere ferromagnetische Metallimplantate. Es wurden nur Patienten mit einem Körpergewicht von unter $125 \mathrm{~kg}$ untersucht, da Patienten mit großem Bauchumfang nicht in das verwendete MRT-Gerät (Innendurchmesser $60 \mathrm{~cm}$ ) passten. Die Untersuchung wurde von der Ethikkommission der UMG genehmigt (Antrag 11/9/11 und Amendement vom 23.10.2013). Alle Patienten erklärten nach einem ausführlichen Aufklärungsgespräch schriftlich ihr Einverständnis zur Teilnahme an der Studie und zur Verwendung ihrer anonymisierten Daten.

Alle 30 Patienten durchliefen die gesamte Untersuchung, bestehend aus CINE-MRT, RealTime-MRT und Echokardiographie. Tabelle 1 zeigt die Geschlechterverteilung, Tabelle 2 zeigt weitere demographische Variablen.

Tabelle 1: Absolute und relative Häufigkeiten des Geschlechts der 30 Patienten.

\begin{tabular}{|l|cc|}
\hline Geschlecht & Häufigkeit & Prozent \\
\hline männlich & $19 / 30$ & 63,3 \\
weiblich & $11 / 30$ & 36,7 \\
\hline
\end{tabular}

Tabelle 2: Deskriptive Kenngrößen für die demographischen Variablen.

\begin{tabular}{|c|c|c|c|c|}
\hline Variable & Minimum & Maximum & Mittelwert & Stabw. \\
\hline Alter bei MRT-Untersuchung (J) & 50 & 91 & 68,9 & 9,1 \\
\hline Größe (cm) & 155 & 200 & 174,9 & 11,0 \\
\hline Gewicht (kg) & 50 & 122 & 82,8 & 17,4 \\
\hline BMI & 18,2 & 37,5 & 27,0 & 4,6 \\
\hline
\end{tabular}




\subsection{Magnetresonanztomographie des Herzens}

Die MRT wurde an einem Magnetom mit der Feldstärke 1,5 Tesla (Magnetom Symphonie, Siemens Healthcare, Erlangen, Deutschland) durchgeführt (Abbildung 6a). Nach der Vorbereitung wurden zuerst Übersichtssequenzen und die CINE-SSFP-Sequenzen der klinischen Routine gefahren. Grundlage war ein standardisiertes Scanprotokoll zur Evaluation der linksventrikulären Morphologie und Funktion (Kramer et al. 2013). Zum Schluss wurden die Real-Time-Aufnahmen gewonnen. Es wurde kein Kontrastmittel verabreicht. Eine Übersicht über das Untersuchungsprotokoll gibt Abbildung 7.

\subsubsection{MRT-Vorbereitung}

Der Untersuchungsablauf erfolgte wie routinemäßig üblich: Rückenlage, Kopf voran, Gehörschutz und Kommunikation über Lautsprecher mit dem MRT-Vorraum. Besonders wichtig war das korrekte Anlegen des Vektor-Elektrokardiogramms, da die gute EKGAbleitung für die korrekte Berechnung der CINE-SSFP-Aufnahmen wichtig war. Zur Detektion des MR-Signals befanden sich acht Spulenelemente im Tisch des Gerätes, sechs weitere Elemente bildeten die Körper-Array-Spule, die ohne Hautkontakt auf den Thorax aufgelegt und fixiert wurde (Abbildung 6b). Abbildung 6c zeigt einen Patienten im Magnetom.
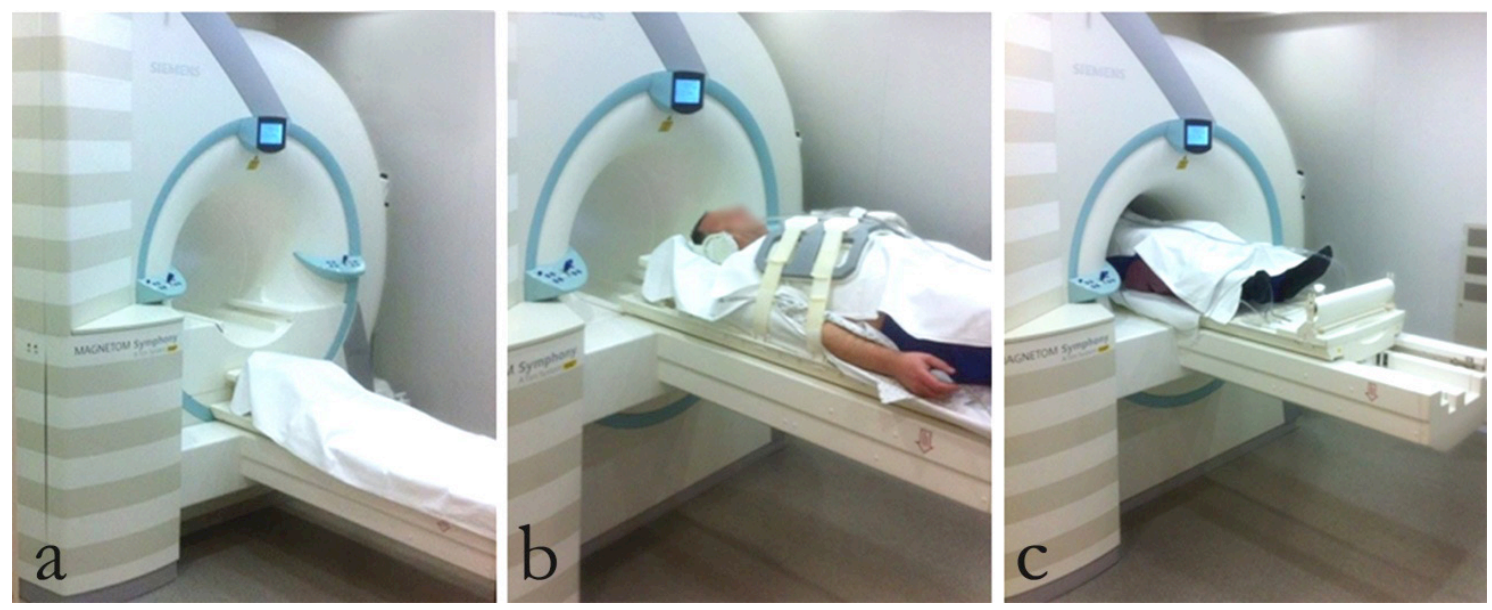

Abbildung 6: Vorbereitung eines Patienten auf die MRT-Untersuchung.

Das Magnetom Symphonie der UMG (a); ein vollständig vorbereiteter Patient mit Gehörschutz, Alarmball und Körper-Array-Spule, die sich auf dem Thorax befindet (b); der Patient im Gerät (c). 


\section{Protokoll der MRT-Untersuchung mit CINE- und Real-Time-SSFP}

\section{1. Übersichtssequenzen}

- True-fast-imaging-with-steady-state-free-precession(TrueFISP)-Localizer

- transversalehalf-fourier-acquisition-single-shot-turbo-spinecho-(HASTE)-Sequenz

- koronare HASTE-Sequenz

- $\quad 2 \mathrm{CV}$

- $\quad 4 \mathrm{CV}$

- SA-Stapel als TrueFISP

2. Standard-Akquisition (CINE-SSFP)

- $\quad 4 \mathrm{CV}$

- $2 \mathrm{CV}$

- $\quad 3 \mathrm{CV}$

- SA-Stapel

3. Real-Time-Akquisition

- $\quad 4 \mathrm{CV}$

- $2 \mathrm{CV}$

- $\quad 3 \mathrm{CV}$

- SA-Stapel

Abbildung 7: Übersicht über das MRT-Untersuchungsprotokoll.

\subsection{2 Übersichtssequenzen}

Als Übersichtssequenzen dienten ein TrueFISP-Localizer (Fuchs et al. 2003) sowie eine transversale und eine koronare HASTE-Sequenz nach Hoffmann et al. (2000). Weiterhin wurden zur Planung ein 2CV, ein 4CV und ein SA-Stapel als TrueFISP aufgenommen. Die Übersichtssequenzen dienten der Orientierung und der Planung der in die Auswertung einfließenden Blicke.

\subsubsection{CINE-MRT}

Für die CINE-SSFP-Aufnahmen mussten die Patienten während der Messung die Luft endexspiratorisch anhalten. Das EKG-Gating erfolgte retrospektiv. Jede CINE-Sequenz enthielt 25 Einzelbilder (Schichtdicke $8 \mathrm{~mm}$, Schichtlücke $0,8 \mathrm{~mm}$ ). Das Field-of-view 
wurde der Herz- und Patientengröße angepasst, um Einfaltungen zu vermeiden. Die zeitliche Auflösung änderte sich in der CINE-SSFP mit der Herzfrequenz des Patienten. Es handelt sich um einen errechneten Wert für die zeitliche Auflösung der interpolierten Bildserie, der keine Aussage über die Geschwindigkeit der Bildakquisition macht. Alle Scanparameter sind in Tabelle 3 aufgeführt.

Die Schichtführung erfolgte - wie für die morphologische Beurteilung und funktionelle Analyse üblich - nach Kramer et al. (2013). Begonnen wurde mit dem 4CV, 2CV und 3CV. Anschließend wurde ein SA-Stapel aus 16 Einzelschichten aufgenommen, der die gesamte Kammer abdeckte und somit alle Wandabschnitte des linken Ventrikels dargestellte. Jede Einzelschicht wurde separat gefahren, um zu langes Luftanhalten zu vermeiden.

\subsubsection{Real-Time-MRT}

Für die Real-Time-SSFP-Sequenzen wurden die Schichtpositionen von den CINE-SSFPSequenzen übernommen. Im Gegensatz zur CINE-SSFP erfolgten die Messungen unter ruhiger Atmung. Die Scanparameter sind in Tabelle 3 aufgeführt.

$4 \mathrm{CV}, 2 \mathrm{CV}$ und 3CV wurden aufgenommen. Es wurden so viele SA-Schnitte gescannt, dass der gesamte linke Ventrikel erfasst wurde. Anschließend wurde daraus ein Stapel zusammengefasst, der alle für die Auswertung relevanten Schichten enthielt.

Tabelle 3: Parameter für die Bildakquisition der CINE- und Real-Time-Bilder.

\begin{tabular}{|c|c|c|}
\hline Parameter & CINE & Real-Time \\
\hline Bildanzahl & 25 & 344 \\
\hline Schichtdicke (mm) & 8 & 8 \\
\hline Field-of-view (mm) & $\begin{array}{c}380 \times 285 \\
\text { (an Patient angepasst) }\end{array}$ & $256 \times 256$ \\
\hline Bildauflösung (Pixel) & $\begin{array}{c}256 \times 192 \\
\text { (an Patient angepasst) }\end{array}$ & $144 \times 144$ \\
\hline Flipwinkel & $64^{\circ}$ & $55^{\circ}$ \\
\hline Echozeit (ms) & 1,36 & 1,54 \\
\hline Repetitionszeit (ms) & 38,4 & 3,08 \\
\hline Zeitliche Auflösung (ms) & $\begin{array}{c}16,56 \text { bis } 62,2 \\
\text { (abhängig von } \mathrm{HF} \text { ) }\end{array}$ & 40,67 \\
\hline
\end{tabular}




\subsection{Transthorakale Echokardiographie}

Die Echokardiographien wurden von einem Untersucher mit über 10 Jahren Erfahrung in der Echokardiographie an einem iE33 Ultraschallsystem (Koninklijke Philips Elecronics N.V., Amsterdam, Niederlande) mit dem Schallkopf X5-1 durchgeführt. Im 2D-Modus wurden EKG-getriggert von jeder Schnittebene zehn Herzschläge aufgenommen. Die linksparasternale kurze Achse (entspricht SA) wurde auf Klappenebene, in Höhe der Kammermitte und am Apex aufgenommen. Aus der apikalen Schallposition heraus wurden $4 \mathrm{CV}, 2 \mathrm{CV}$ und 3CV aufgenommen, die die gleichen anatomischen Strukturen darstellen wie für die MRT-Schnittebenen beschrieben.

\subsection{Qualitätsanalyse der MRT-Bilder}

Als Grundlage diente das Bildqualitätsscoring von Voit et al. (2013), das für diese Studie weiterentwickelt wurde (Tabelle 4). Sowohl die CINE- als auch die Real-Time-SSFPBildserien wurden von zwei erfahrenen Untersuchern, „Untersucher 1“ mit sechs Jahren und „Untersucher 2“ mit zwei Jahren Erfahrung in der Kardio-MRT-Befundung, an einer Centricity PACS Radiology RA1000 Workstation (GE Health Care, Little Chalfont, Großbritannien) als laufendes Video betrachtet und bewertet. Die Untersucher waren gegeneinander verblindet, die Bildreihenfolge war zufällig und anonymisiert. Folgende Bildserien (sowohl CINE- als auch Real-Time-SSFP) wurden betrachtet:

- $2 \mathrm{CV}, 3 \mathrm{CV}, 4 \mathrm{CV}$

- SA-Schnitt auf Höhe der Herzbasis (SA basis)

- SA-Schnitt auf Höhe der Kammermitte (SA mid)

- SA-Schnitt auf Höhe der Herzspitze (SA apex)

Zunächst erfolgte eine Bewertung des Gesamteindrucks („Beurteilung der Diagnosequalität aus Erfahrung“). Dann wurden die Einzelkriterien „Abgrenzbarkeit Septum“ (im 2CV „Abgrenzbarkeit anteriore Wand“), „Abgrenzbarkeit Seitenwand“ (im 2CV „Abgrenzbarkeit inferiore Wand“) sowie „Sichtbarkeit und Abgrenzbarkeit der Papillarmuskeln“ zur genaueren Evaluation der Bildschärfe bewertet. Die Bewertung der Papillarmuskeln erfolgte nur in SA mid, da sie nur dort regelmäßig abgrenzbar sind. Entscheidend bei der Bewertung war die Beurteilbarkeit des linken Ventrikels. 
Tabelle 4: Score zur Beurteilung der Diagnosequalität.

\begin{tabular}{|c|c|c|c|}
\hline & Gesamteindruck & $\begin{array}{l}\text { Bildschärfe (Sichtbarkeit } \\
\text { einzelnen Strukturen) }\end{array}$ & und Abgrenzbarkeit der \\
\hline & $\begin{array}{c}\text { Beurteilung der } \\
\text { Diagnosequalität aus } \\
\text { Erfahrung }\end{array}$ & $\begin{array}{l}\text { Septum/anteriore Wand } \\
\text { Seitenwand/inferiore Wand }\end{array}$ & Papillarmuskeln \\
\hline 0 & Keine Diagnosequalität & Keine Abgrenzung möglich & $\begin{array}{l}\text { Nicht klar vom Blut } \\
\text { abzugrenzen }\end{array}$ \\
\hline 1 & $\begin{array}{c}\text { Reduzierte } \\
\text { Diagnosequalität }\end{array}$ & $\begin{array}{l}\text { Schwer abzugrenzen wegen } \\
\text { deutlicher Unschärfe }\end{array}$ & $\begin{array}{l}\text { Nur schwer vom Blut } \\
\text { abzugrenzen }\end{array}$ \\
\hline 2 & $\begin{array}{c}\text { Noch beurteilbar mit vielen } \\
\text { Artefakten }\end{array}$ & $\begin{array}{l}\text { Abgrenzbar trotz } \\
\text { Unschärfe }\end{array}$ & $\begin{array}{l}\text { Struktur erkennbar, } \\
\text { Grenzen schwer zu } \\
\text { definieren }\end{array}$ \\
\hline 3 & $\begin{array}{l}\text { Hohe Diagnosequalität } \\
\text { trotz einiger Artefakte }\end{array}$ & $\begin{array}{c}\text { Abgrenzbar trotz leichter } \\
\text { Unschärfe }\end{array}$ & $\begin{array}{l}\text { Struktur erkennbar, } \\
\text { Abgrenzen möglich }\end{array}$ \\
\hline 4 & Optimale Diagnosequalität & Sichere Abgrenzbarkeit & $\begin{array}{c}\text { Sicher abgrenzbar gegen } \\
\text { Blut }\end{array}$ \\
\hline
\end{tabular}

\subsection{Analyse der linksventrikulären Funktion anhand der MRT- Aufnahmen}

Für SA-Stapel und 4CV fand eine Analyse der linksventrikulären Funktion in CINE- und Real-Time-SSFP statt. Berücksichtigt wurden dabei nur Bildserien, bei denen eine sinnvolle Segmentation möglich war. Hatte ein 4CV oder ein SA-Stapel von beiden Untersuchern bei der Qualitätsanalyse im Gesamteindruck keine Note höher als Zwei und mindestens einmal eine Null oder Eins erhalten, wurde dieser von der Auswertung der funktionellen Parameter ausgeschlossen.

Zur Auswertung der MRT-Sequenzen wurde die Segmentationssoftware QMass MR (7.4.14.0 - Real-Time Prototyp, Medis medical imaging systems bv, Leiden, Niederlande) verwendet. Alle Segmentationen erfolgten manuell und in Anlehnung an die aktuellen Leitlinien (Schulz-Menger et al. 2013) im Konsens mit Untersucher 1 oder Untersucher 2.

Im CINE-SSFP-SA-Stapel wurden Endokard und Epikard des linken Ventrikels in ED und ES segmentiert (Abbildung 8a-c). Die ED war das Bild mit dem nach visueller Betrachtung größten, die ES das mit dem kleinsten linksventrikulären Blutvolumen auf midventrikulärer Ebene. Basal wurde mit der Schicht begonnen, bei der in beiden Herzphasen noch ein vollständig umschlossener Myokardring erkennbar war. Dies sollte den Vergleich zur Real-Time-SSFP erleichtern, in der sich die Position der Klappenebene 
von Schlag zu Schlag ändern kann. Apikal wurde die Schicht eingeschlossen, in der noch klar Myokard zu erkennen war.

Gemäß Richtlinien wurden anschließend in allen eingeschlossenen Schichten in ED und ES Konturen eingezeichnet, die Endokard und Epikard des linken Ventrikels markierten. Die Papillarmuskeln wurden gesondert segmentiert und damit der myokardialen Masse zugeordnet. Auf Grundlage dieser Segmentation wurden folgende Parameter berechnet, die zusätzlich auf die nach Du Bois und Du Bois (1916) ermittelte Körperoberfläche des Patienten bezogen wurden:

- Myokardiale Masse (MM in $\mathrm{g}$ und MM-Index in $\mathrm{g} / \mathrm{m}^{2}$ )

- Enddiastolisches Volumen (EDV in $\mathrm{ml}$ und EDV-Index in $\mathrm{ml} / \mathrm{m}^{2}$ )

- Endsystolisches Volumen (ESV in $\mathrm{ml}$ und ESV-Index in $\mathrm{ml} / \mathrm{m}^{2}$ )

- Schlagvolumen (SV in $\mathrm{ml}$ und SV-Index in $\mathrm{ml} / \mathrm{m}^{2}$ )

- Ejektionsfraktion (\%)

- Herzzeitvolumen (HZV in $1 /$ min und HZV-Index in $1 / \mathrm{min} / \mathrm{m}^{2}$ )

Im CINE-SSFP-4CV erfolgte die Segmentation nach dem gleichen Prinzip, jedoch nur in einer Schicht (Abbildung 8d und e); die Papillarmuskeln wurden hier als Bestandteil des Blutpools betrachtet, da ihr Volumen auf dem zweidimensionalen Schnitt nicht genau bestimmbar ist. 

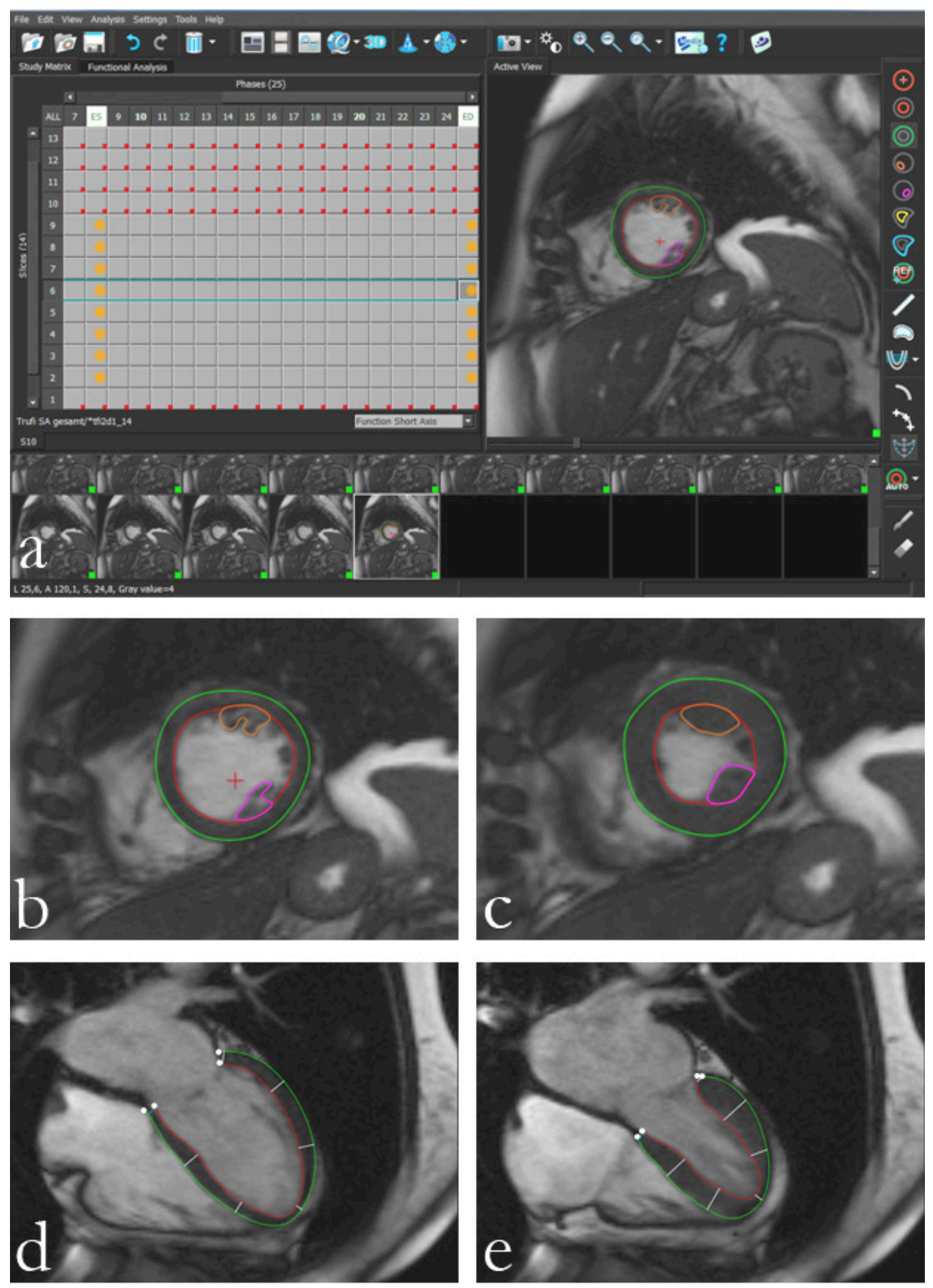

Abbildung 8: Segmentation der CINE-SSFP-Aufnahmen mit QMass.

ED und ES wurden automatisch untereinandergelegt (a). In beiden Herzphasen wurden Endokard (rot) und Epikard (grün) segmentiert (b / c = ED / ES in SA mid; d /e: ED / ES im 4CV), Papillarmuskeln (orange/violett) nur in SA mid. 
Beim Real-Time-SSFP-SA-Stapel besteht jede Schnittebene aus 344 konsekutiven Einzelbildern mit einer zeitlichen Auflösung von 40,67 ms. Die Anzahl der darin enthaltenen Einzelschläge ist von der Herzfrequenz des Patienten abhängig. Die Segmentation erfolgte prinzipiell analog zur Segmentation des CINE-SSFP-SA-Stapels, jedoch über sieben konsekutive Schläge. In jeder Schicht wurden die dritte eindeutig identifizierbare ED und die dazugehörige ES segmentiert. ED war das Bild, auf dem der linke Ventrikel eine visuell größere Querschnittsfläche aufwies als auf dem davor und dahinter liegenden Bild. Das Bild, auf dem der linke Ventrikel eine visuell kleinere Querschnittsfläche aufwies als auf dem davor und dahinter liegenden Bild, wurde als ES definiert. Anschließend wurde aus der Segmentation über alle Schichten ein SA-Stapel zusammengesetzt, aus dem die funktionellen Parameter berechnet wurden (Abbildung 9a-c). Dieser Vorgang wurde für die folgenden sechs Herzschläge wiederholt. Durch dieses Vorgehen wurden auch ventrikuläre Extrasystolen in die Auswertung eingeschlossen. Pro Real-Time-SSFP-SA-Stapel ergaben sich für jeden funktionellen Parameter sieben Werte, die anschließend gemittelt wurden. So war ein guter Vergleich mit der CINE-SSFP möglich, bei der Informationen aus etwa zehn bis 20 Herzschlägen in die Auswertung eingehen. Da QMass die Herzfrequenz aufgrund fehlender EKG-Triggerung bei den RealTime-Bildern nicht automatisch berechnen konnte, wurde die Schlagdauer als Produkt aus Anzahl der Bilder pro Schlag (vom Bild der ED bis zum Bild vor der nächsten ED) und der zeitlichen Auflösung ermittelt. Die so errechnete Dauer jedes einzelnen Schlages wurde auch verwendet, um eine Analyse der Verteilung der Schlagdauer pro Patient durchzuführen. Außerdem konnte damit für jeden Einzelschlag eine hypothetische Herzfrequenz (HF) ermittelt werden. Das HZV wurde dann errechnet als Produkt aus dem vom Programm berechneten SV und der über alle Schläge eines Stapels gemittelten HF.

Die Segmentation des Real-Time-SSFP-4CV erfolgte nach dem gleichen Prinzip wie die Segmentation des CINE-SSFP-4CV (Abbildung 9d und e). Jedoch wurden auch hier ab der dritten eindeutig zu identifizierenden ED sieben konsekutive Herzschläge segmentiert. Für jeden der sieben segmentierten Herzschläge wurden die funktionellen Parameter berechnet. Schlagdauer, HF und HZV mussten auch hier gesondert ermittelt werden. 

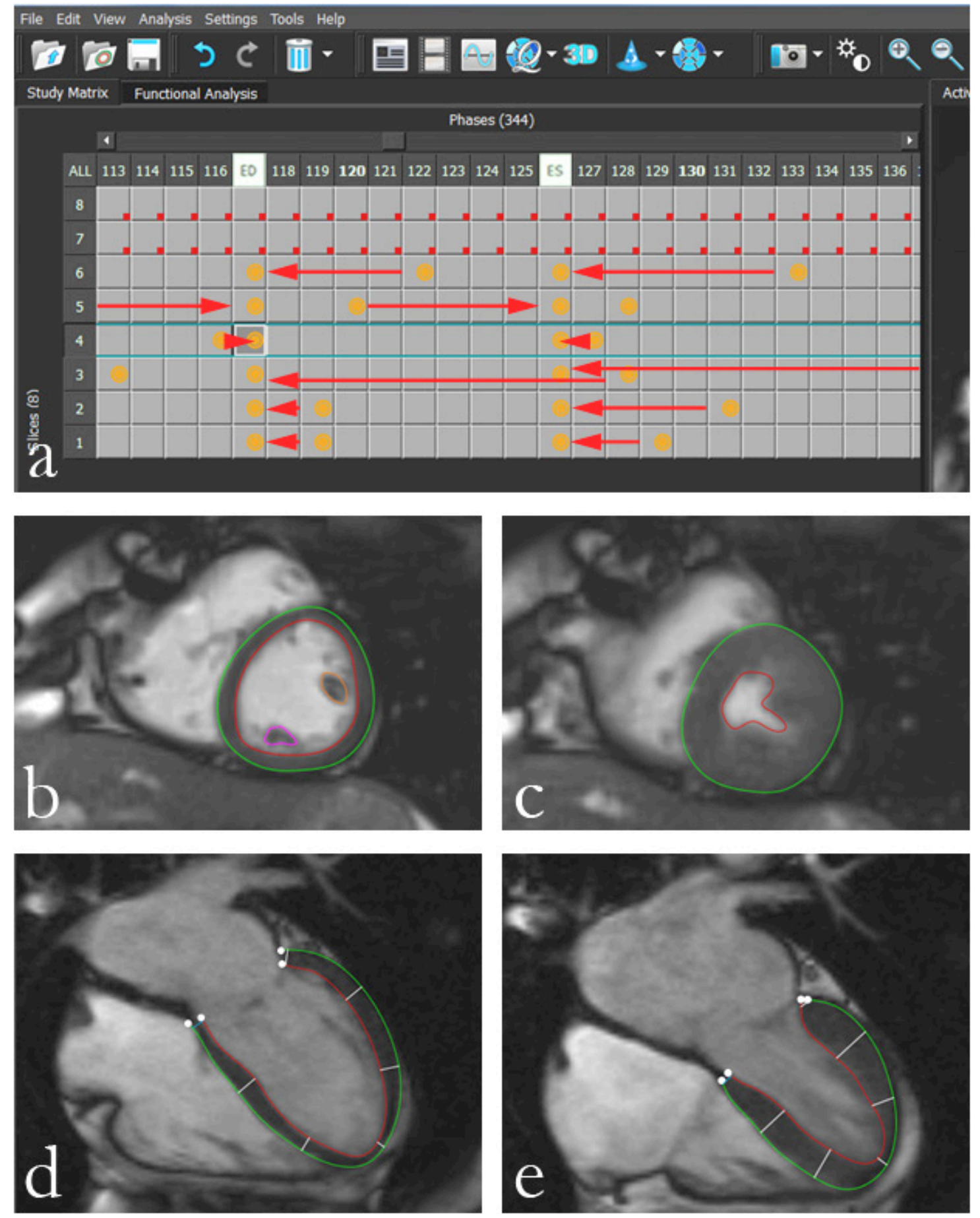

Abbildung 9: Segmentation der Real-Time-SSFP mit QMass.

Nachdem in allen Schichten segmentiert worden war, wurden die Konturen zu je einem Stapel von ED- und ES-Bildern gruppiert, damit eine 3D-Volumetrie erfolgen konnte. In Zeile 6, in der die Herzbasis dargestellt ist, liegt das ED-Bild in Spalte 122. Die dort entstandene Kontur wurde wie die Konturen aller anderen ED-Bilder in Spalte 117 kopiert. So entstand ein dreidimensionaler Konturstapel. Die Konturen aller ES-Bilder - in Zeile 6 z.B. aus Spalte 133 - wurden in Spalte 127 kopiert (a). Auch hier wurden in beiden Herzphasen Endokard (rot), und Epikard (grün) segmentiert $(\mathbf{b} / \mathbf{c}=\mathrm{ED} / \mathrm{ES}$ in $\mathrm{SA} \mathrm{mid} ; \mathbf{d} / \mathbf{e}$ : $\mathrm{ED} / \mathrm{ES} \mathrm{im} 4 \mathrm{CV})$. Die Segmentation der Papillarmuskeln (orange/violett) erfolgte nur in SA mid. 


\subsection{Analyse der linksventrikulären Funktion anhand der Echokardiographie}

Die Echokardiographien wurden mit Image Arena (Version 4.6 Build 4.6.3.9, TomTec Imaging Systems, Unterschleisheim, Deutschland) ausgewertet. Im apikalen 2- und 4CV (Abbildung 10) wurden aus zehn aufgenommenen Schlägen jeweils fünf ausgewählt, in denen das Endokard von ED und ES segmentiert wurde. Bei der verwendeten Software konnten nicht mehr als fünf Schläge pro Bildserie für die Auswertung berücksichtigt werden. Zur Berechnung der funktionellen Parameter wurde die biplane Scheibchensummationsmethode nach Simpson verwendet. Sie gilt als Methode der Wahl für die Bestimmung funktioneller Parameter in der 2D-Echokardiographie (Lang et al. 2005). Werte für die folgenden funktionellen Parameter wurden gemittelt über zehn Schläge (fünf Schläge 4CV, fünf Schläge 2CV) erhoben: EDV (ml), ESV (ml), SV (ml), HZV (l/min) und EF (\%). Zur Berechnung des HZV diente die aus beiden Schnitten gemittelte Herzfrequenz.
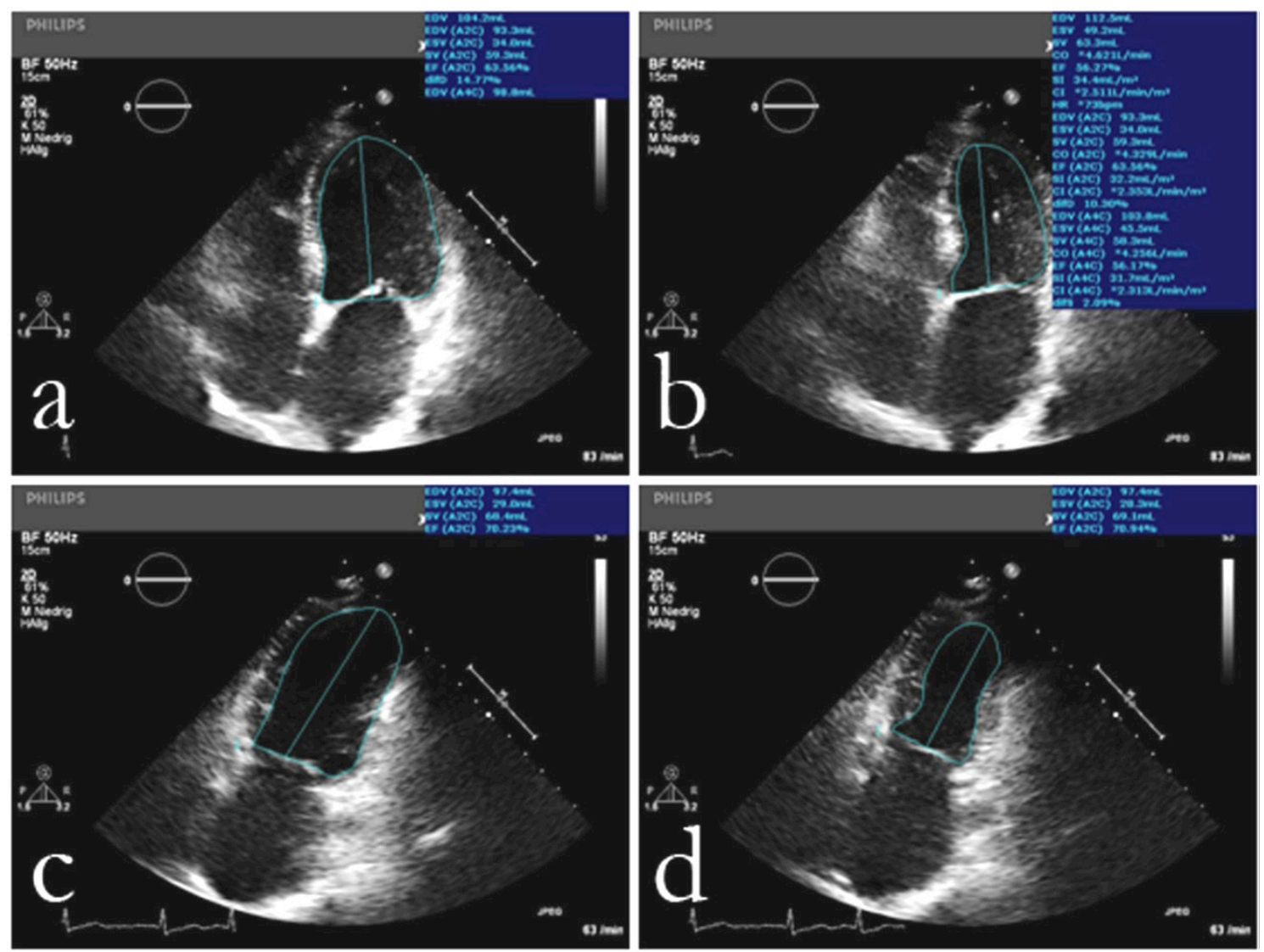

Abbildung 10: Echokardiographie - Segmentation enddiastolisch und endsystolisch.

Die blaue Kontur stellt die Segmentation des Endokards im linken Ventrikel dar, die manuell erfolgte (4CV-ED: a, 4CV-ES: b, 2CV-ED: c, 2CV-ES: d). 


\subsection{Statistische Methoden}

\subsubsection{Analyse der Verteilung der Schlagdauer pro Patient}

Alle im Rahmen der funktionellen Analyse mithilfe der Real-Time-SSFP evaluierten Schläge wurden zur Analyse der Verteilung der Schlagdauer pro Patient herangezogen. Für jeden Patienten wurden mittlere, mediane, minimale und maximale Schlagdauer sowie Standardabweichung ermittelt. Zusätzlich wurden Histogramme erstellt, um die Verteilung pro Patient zu visualisieren.

\subsubsection{Qualitätsanalyse}

Für beide Untersucher fand ein Vergleich zwischen der Bewertung der CINE-SSFPAufnahmen und der Real-Time-SSFP-Sequenzen statt. Der Vergleich erfolgte einzeln für jedes der Bewertungskriterien in 2-, 3- und 4CV sowie in SA basis, mid und apex, für das Kriterium „Sichtbarkeit und Abgrenzbarkeit der Papillarmuskeln“ nur in SA mid, da diese nur in den mittleren Achsen zuverlässig abgebildet werden. Im 2CV wurden statt für Septum und Seitenwand die Bewertungen für die auf dieser Schnittebene erkennbare anteriore bzw. posteriore Wand verglichen.

Zur graphischen Darstellung der Ergebnisse wurden Bubble-Plots verwendet. Dabei handelt es sich um Streudiagramme, bei denen auf der x-Achse die Notenskala von null bis vier für die CINE-SSFP und auf der $y$-Achse die Notenskala von null bis vier für die RealTime-SSFP aufgetragen wurde. Das Diagramm enthält die Funktionsgerade $\mathrm{y}=\mathrm{x}$. Punkte auf dieser Geraden symbolisieren gleiche Bewertung in CINE und Real-Time. Punkte unterhalb der Geraden symbolisieren eine höhere Bewertung für die CINE-, Punkte oberhalb der Geraden eine höhere Note für die Real-Time-SSFP. Die Größe der Symbole gibt Auskunft über die Häufigkeit der Notenkombination. Zur Veranschaulichung zeigt Abbildung 11 eine Bewertung des 4CV. 


\section{CV Gesamteindruck - Untersucher 1}

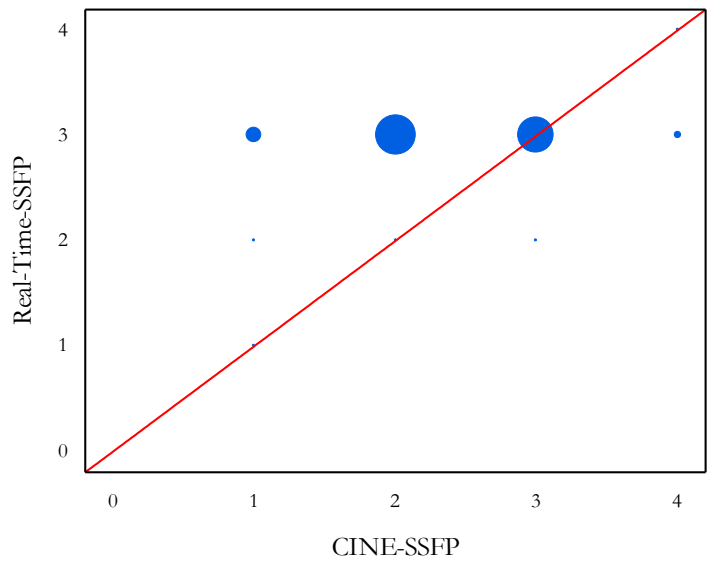

Abbildung 11: Beispiel für einen Bubble-Plot.

Vergleich CINE-SSFP- gegen Real-Time-SSFP-Gesamteindruck im 4CV bei Untersucher 1. Die Größe der blauen Punkte symbolisiert die Häufigkeit einer Notenkombination. Punkte auf der Funktionsgeraden $\mathrm{y}=\mathrm{x}$ bedeuten gleiche Bewertung, Punkte darüber eine bessere Bewertung der Real-Time-SSFP und Punkte darunter eine bessere Bewertung der CINE-SSFP.

Für alle Paarvergleiche wurde außerdem der Wilcoxon-matched-Pairs-Test (Lehmann und D'Abrera 1998) durchgeführt mit der Fragestellung, ob sich die beiden verglichenen Beurteilungen signifikant voneinander unterscheiden. Die Nullhypothese war also, dass es keinen Unterschied zwischen den beiden verglichenen Beurteilungen gibt. Das Signifikanzniveau wurde auf $\mathrm{p}=0,05$ festgelegt, sodass von einem signifikanten Unterschied zwischen den Beurteilungen gesprochen werden kann, wenn p einen Wert kleiner als 0,05 annimmt. Um festzustellen, in welchem Fall höhere und in welchem niedrigere Noten vergeben wurden, muss der zugehörige Bubble-Plot betrachtet werden.

Zur Evaluation der Interrater-Variabilität wurden die Bewertungen der beiden Untersucher mithilfe des Wilcoxon-matched-pairs-Tests auf einen signifikanten Unterschied geprüft. In Bubble-Plots wurden die Bewertungen der beiden Untersucher gegeneinander aufgetragen um bei Vorliegen eines signifikanten Unterschieds festzustellen, welcher Untersucher höhere Noten vergeben hat.

Beide Untersucher bewerteten die Aufnahmen von 15 zufällig ausgewählten Patienten (50\% aller Patienten) nach mindestens 30 Tagen ein zweites Mal. Dies diente zur Überprüfung der Intrarater-Variabilität. Auch für diesen Vergleich wurden der Wilcoxonmatched-pairs-Test und Bubble-Plots verwendet.

\subsubsection{Linksventrikuläre Funktion}

Grundlage der statistischen Auswertung bildeten die Ergebnisse der CINE-Segmentation und bei der Real-Time-Segmentation die Ergebnisse der Mittelung über sieben SA-Stapel bzw. sieben Schläge im 4CV. Für den Vergleich mit der Echokardiographie wurden die 
über fünf Schläge gemittelten Ergebnisse der Simpson-biplan-Methode verwendet. Zum Methodenvergleich diente die Bland-Altman-Analyse (Bland und Altman 1999). Wichtige Parameter der Bland-Altman-Analyse sind die Verzerrung, die die mittlere Differenz der mit beiden Methoden erhobenen Werte angibt, und die Übereinstimmungsgrenzen, die ein Maß für die Streuung der Werte sind. Es wurden für alle erhobenen Parameter folgende Paarvergleiche durchgeführt:

- CINE-SSFP- vs. Real-Time-SSFP- SA-Stapel

- CINE-SSFP- vs. Real-Time-SSFP-4CV

Die folgenden Paarvergleiche wurden für alle erhobenen Parameter außer für die myokardiale Masse durchgeführt, da diese nicht echokardiographisch ermittelt wurde:

- Echokardiographie vs. CINE-SSFP-4CV

- Echokardiographie vs. Real-Time-SSFP-4CV

In der Echokardiographie wird bei VHF zur Beurteilung der linksventrikulären Funktion eine Mindestzahl von drei Schlägen empfohlen (Evangelista et al. 2008). In der verwendeten Software zur Auswertung der Echokardiographien war die Verrechnung von maximal fünf Schlägen möglich. Daher wurden die Ergebnisse der Segmentation bei einer Mittelung über sieben Schläge mit denen der Mittelung über fünf bzw. drei Schläge im Real-Time-SSFP-4CV verglichen. Dadurch, dass der zweidimensionale 4CV verwendet wurde, handelt es sich tatsächlich um eine Mittelung aus einzelnen Schlägen. Dies sollte dazu dienen, herauszufinden, ob sich auch mit weniger Bildmaterial und Segmentationsaufwand zuverlässige Aussagen über die linksventrikuläre Funktion des Arrhythmikers treffen lassen. Unter Anwendung der Bland-Altman-Analyse wurde die Mittelung über sieben Schläge mit den beiden anderen Mittelungen verglichen.

Da es im Real-Time-SSFP-4CV möglich war, für jeden Einzelschlag die funktionellen Parameter zu erheben, handelt es sich hier um eine echte Schlag-zu-Schlag-Analyse. Für Schlaglänge und EF wurde bei jedem Patienten die Spannweite über sieben Schläge berechnet. 


\section{Ergebnisse}

MRT-Aufnahmen in CINE und Real-Time wurden von allen 30 Patienten gewonnen. In der Echokardiographie liegt bei 28 Patienten eine vollständige Untersuchung vor. Bei zwei Patienten konnte aufgrund der anatomischen Gegebenheiten kein brauchbares Schallfenster eingestellt werden. Bei einem Patienten musste die Untersuchung wiederholt werden, da beim ersten Mal aufgrund eines Anwenderfehlers MRT-Daten fehlten.

\subsection{Analyse der Verteilung der Schlagdauer}

Die meisten Patienten waren normofrequent; drei Patienten waren tachykard (mittlere Schlagdauer < $600 \mathrm{~ms}$ ), drei waren bradykard (mittlere Schlagdauer > $1000 \mathrm{~ms}$ ). Die mittlere Schlagdauer lag zwischen 468 bis 1193 ms, der Median zwischen 447 und 1139 ms (Tabelle 5). Intraindividuell gab es starke Schwankungen, wie an der Spannweite (Differenz zw. Minimum und Maximum) und an den Histogrammen (Abbildung 12) zu sehen ist. Das während des MRT-Scans mitlaufende EKG zeigte bei allen Patienten VHF.

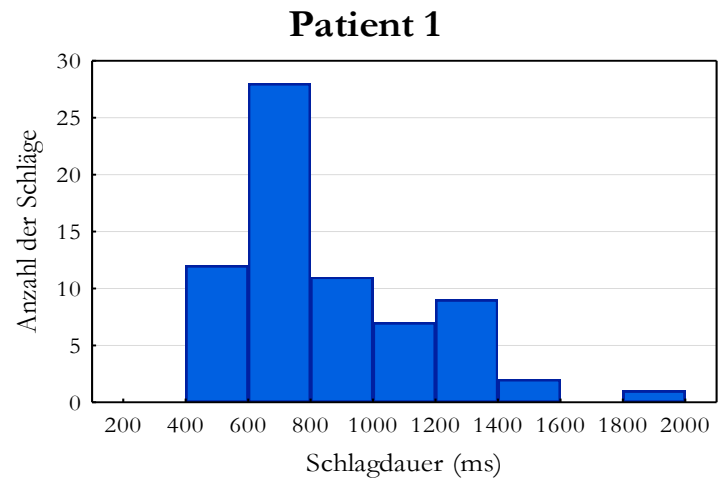

Patient 13

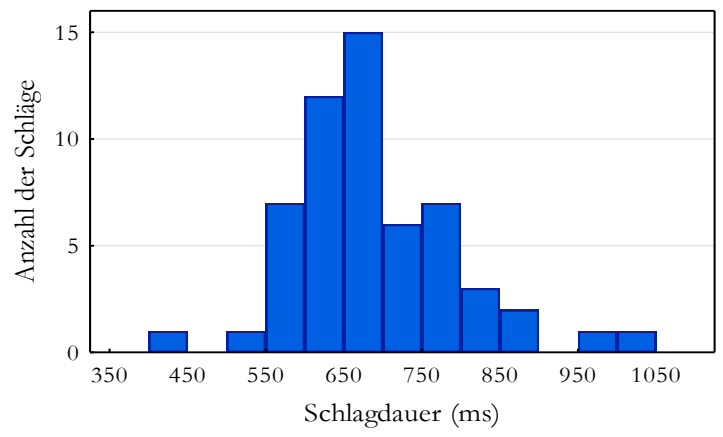

Patient 6

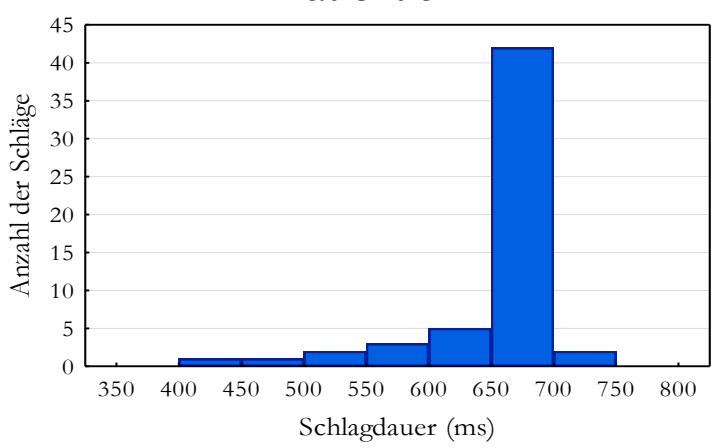

Patient 19

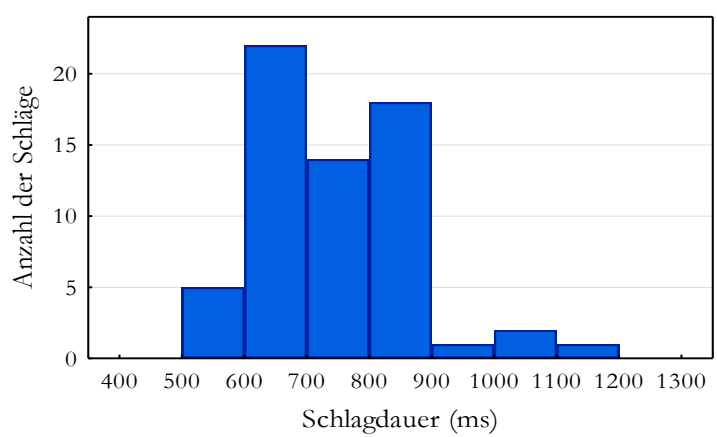

Abbildung 12: Histogramme zur Darstellung der Verteilung der Schlagdauer.

Patient 1 und 13 zeigen eine breite Streuung, während Patient 6 mit einigen deutlichen Abweichungen eine eher rhythmische Herzaktion aufweist. Patient 19 zeigt häufig Schläge mit einer Dauer von 600 bis $900 \mathrm{~ms}$. 
Tabelle 5: Kenngrößen zur Verteilung der Schlagdauer (ms) pro Patient.

\begin{tabular}{|c|c|c|c|c|c|c|c|}
\hline Nr. & $\begin{array}{l}\text { analysierte } \\
\text { Schläge }\end{array}$ & Mittelwert & Median & Minimum & Maximum & Spannweite & Stabw. \\
\hline 1 & 70 & 859 & 773 & 529 & 1830 & 1301 & 277 \\
\hline 2 & 84 & 776 & 773 & 529 & 1220 & 591 & 149 \\
\hline 3 & 63 & 816 & 813 & 529 & 1220 & 691 & 150 \\
\hline 4 & 63 & 468 & 447 & 285 & 854 & 569 & 95 \\
\hline 5 & 56 & 779 & 732 & 529 & 1057 & 528 & 139 \\
\hline 6 & 56 & 649 & 651 & 407 & 732 & 325 & 59 \\
\hline 7 & 56 & 574 & 529 & 325 & 1057 & 732 & 150 \\
\hline 8 & 54 & 1193 & 1078 & 651 & 2603 & 1952 & 455 \\
\hline 9 & 63 & 619 & 569 & 366 & 1301 & 965 & 185 \\
\hline 10 & 56 & 762 & 732 & 529 & 1220 & 691 & 190 \\
\hline 11 & 56 & 664 & 610 & 407 & 1383 & 976 & 176 \\
\hline 12 & 63 & 611 & 569 & 285 & 1220 & 935 & 237 \\
\hline 13 & 56 & 686 & 691 & 447 & 1017 & 570 & 107 \\
\hline 14 & 63 & 946 & 935 & 488 & 1423 & 935 & 189 \\
\hline 15 & 70 & 838 & 773 & 366 & 1667 & 1301 & 244 \\
\hline 16 & 49 & 1092 & 1057 & 772 & 1749 & 977 & 261 \\
\hline 17 & 49 & 706 & 691 & 529 & 1179 & 650 & 127 \\
\hline 18 & 49 & 857 & 854 & 610 & 1220 & 610 & 110 \\
\hline 19 & 63 & 747 & 732 & 529 & 1179 & 650 & 125 \\
\hline 20 & 63 & 987 & 976 & 610 & 1586 & 976 & 218 \\
\hline 21 & 49 & 638 & 610 & 529 & 854 & 325 & 73 \\
\hline 22 & 56 & 651 & 651 & 529 & 813 & 284 & 63 \\
\hline 23 & 56 & 823 & 813 & 610 & 1220 & 610 & 136 \\
\hline 24 & 56 & 757 & 732 & 529 & 1179 & 650 & 135 \\
\hline 25 & 7 & 546 & 529 & 488 & 610 & 122 & 52 \\
\hline 26 & 49 & 893 & 854 & 691 & 1749 & 1058 & 205 \\
\hline 27 & 49 & 1163 & 1139 & 610 & 1993 & 1383 & 282 \\
\hline 28 & 49 & 841 & 854 & 691 & 1017 & 326 & 86 \\
\hline 29 & 56 & 784 & 773 & 488 & 1464 & 976 & 178 \\
\hline 30 & 56 & 749 & 732 & 569 & 1098 & 529 & 120 \\
\hline
\end{tabular}




\subsection{Qualitätsanalyse}

Die MRT-Sequenzen von allen 30 Patienten konnten bewertet werden.

\subsubsection{Gesamteindruck}

Beim Gesamteindruck ging es um eine Beurteilung der Diagnosequalität durch beide Untersucher unabhängig voneinander. CINE-SSFP und Real-Time-SSFP wurden miteinander verglichen.

Für 2-, 3-, und 4CV sowie SA basis und SA mid gab es einen signifikanten Unterschied $(\mathrm{p}<0,05)$ zwischen CINE- und Real-Time-SSFP bei beiden Untersuchern, in SA apex nur bei einem Untersucher (Untersucher 2). Tabelle 6 zeigt die zugehörigen p-Werte (Ergebnisse des Wilcoxon-matched-pairs-Tests).

Die Bubble-Plots (Abbildung 13 und Abbildung 14) zeigen, dass bei Vorliegen eines signifikanten Unterschieds die Real-Time-SSFP tendenziell besser bewertet wurde. Der Bubble-Plot für SA apex bei Untersucher 1 zeigt eine Tendenz zur gleichen oder besseren Bewertung der Real-Time-SSFP. Da die Aussage, welche Methode besser und wie hoch die Qualität bewertet wurde, nur anhand dieser Grafiken abgelesen werden kann, sind sie vollständig abgebildet.

Die Abbildungen 15 bis 17 geben Beispiele für den intraindividuellen Vergleich von MRT-Bildern in CINE und Real-Time in 2CV, 3CV, 4CV und SA mid. Exemplarisch wurden einige enddiastolische und endsystolische Aufnahmen ausgewählt. Es ist zu beachten, dass die Bewertung am bewegten Bild erfolgte.

Tabelle 6: Qualitätsanalyse Gesamteindruck - Ergebnisse des Wilcoxon-matched-pairs-Tests. Signifikante Ergebnisse $(\mathrm{p}<0,05)$ sind fett gedruckt.

\begin{tabular}{|l|cc|}
\hline Schnittebene & p Untersucher 1 & p Untersucher 2 \\
\hline 2 CV & $\mathbf{0 , 0 1 1 1}$ & $\mathbf{0 , 0 1 1 1}$ \\
3 CV & $\mathbf{0 , 0 1 8 7}$ & $<\mathbf{0 , 0 0 0 1}$ \\
4CV & $\mathbf{0 , 0 0 3 6}$ & $\mathbf{0 , 0 0 4 9}$ \\
SA basis & $\mathbf{0 , 0 0 0 5}$ & $<\mathbf{0 , 0 0 0 1}$ \\
SA mid & $\mathbf{0 , 0 0 0 2}$ & $<\mathbf{0 , 0 0 0 1}$ \\
SA apex & 0,0706 & $\mathbf{0 , 0 1 4 4}$ \\
\hline
\end{tabular}


2CV Gesamteindruck - Untersucher 1

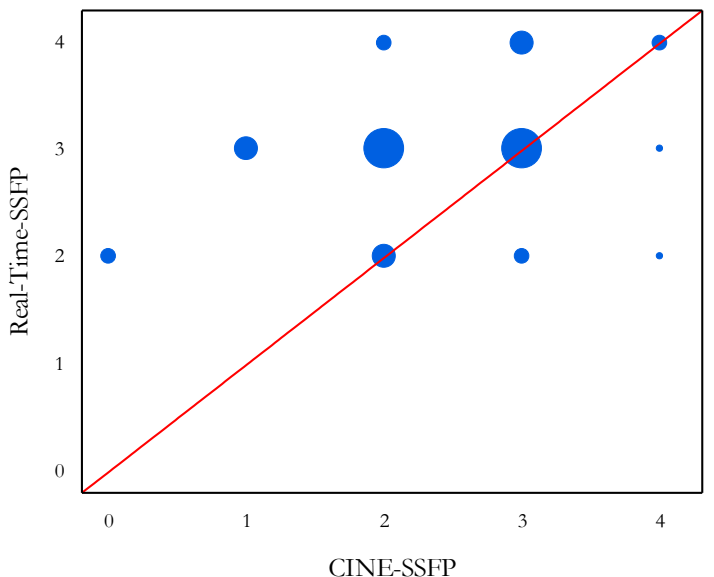

3CV Gesamteindruck - Untersucher 1

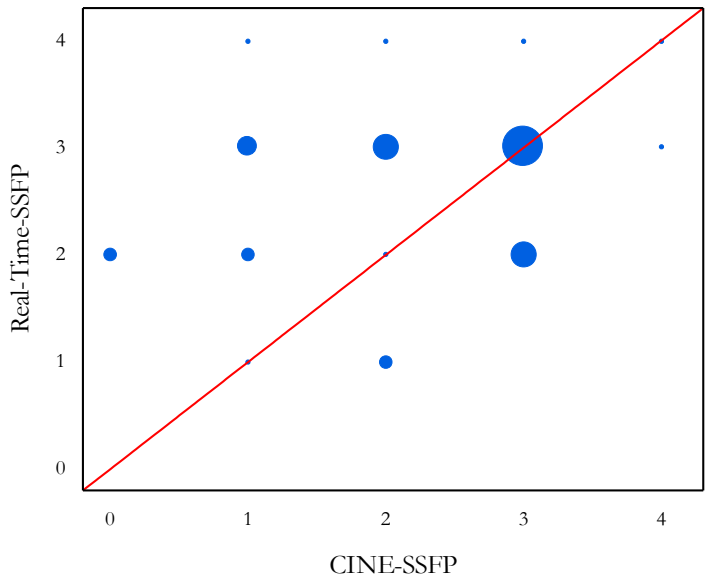

4CV Gesamteindruck - Untersucher 1

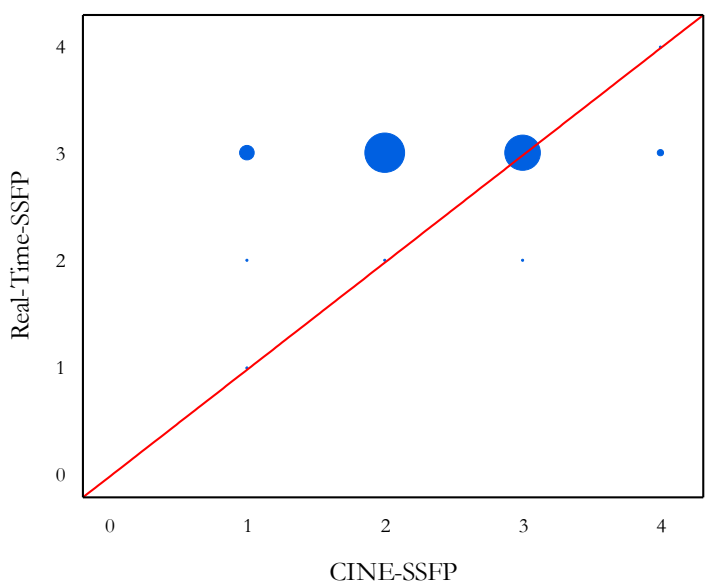

2CV Gesamteindruck - Untersucher 2

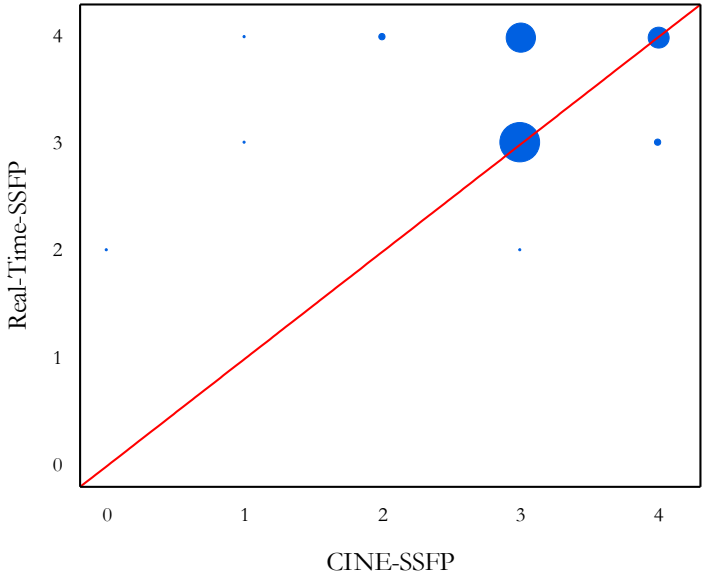

3CV Gesamteindruck - Untersucher 2

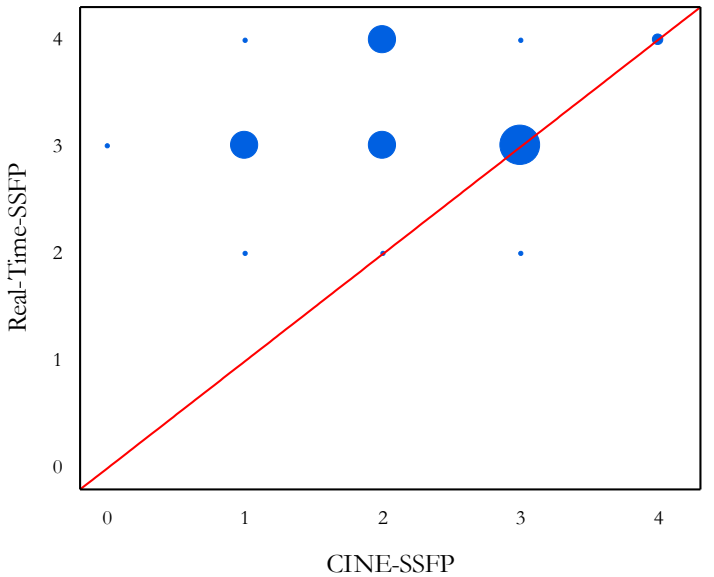

4CV Gesamteindruck - Untersucher 2

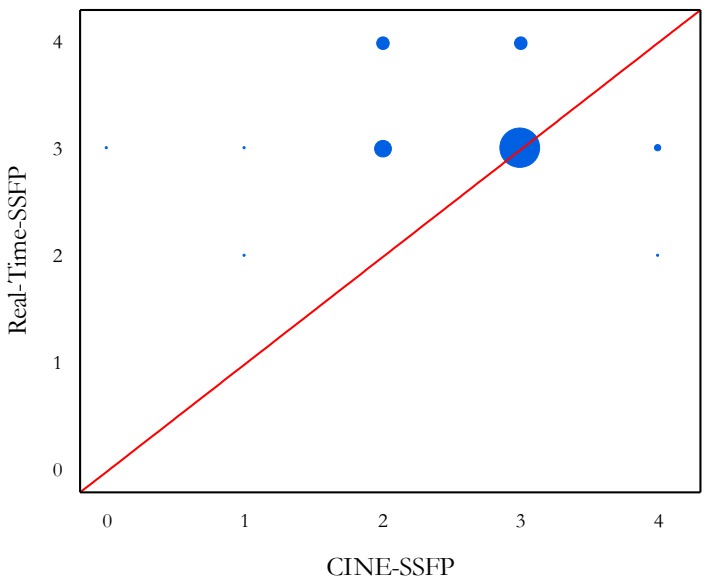

Abbildung 13: Bubble-Plots für die Qualitätsanalyse der langen Achsen im Gesamteindruck. Die Diagramme zeigen den Vergleich der Bewertungen für CINE-SSFP und Real-Time SSFP für 2CV, 3CV und 4CV durch Untersucher 1 (links) und Untersucher 2 (rechts). 
SA basis Gesamteindruck - Untersucher 1

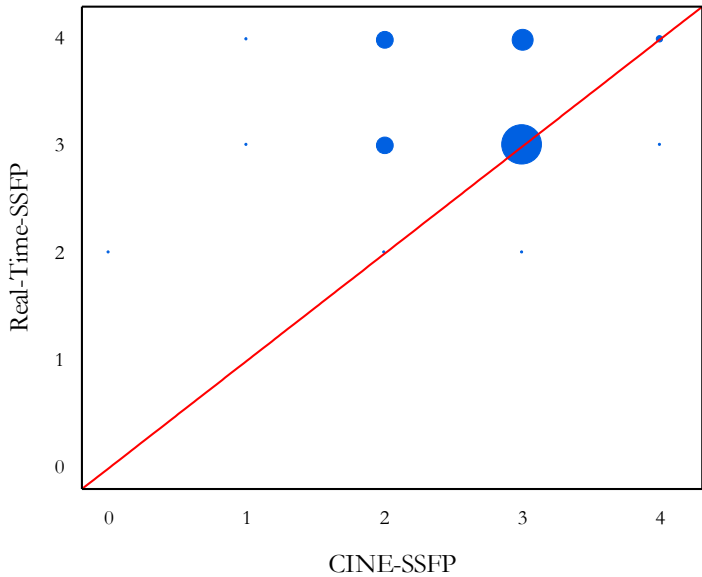

SA mid Gesamteindruck - Untersucher 1

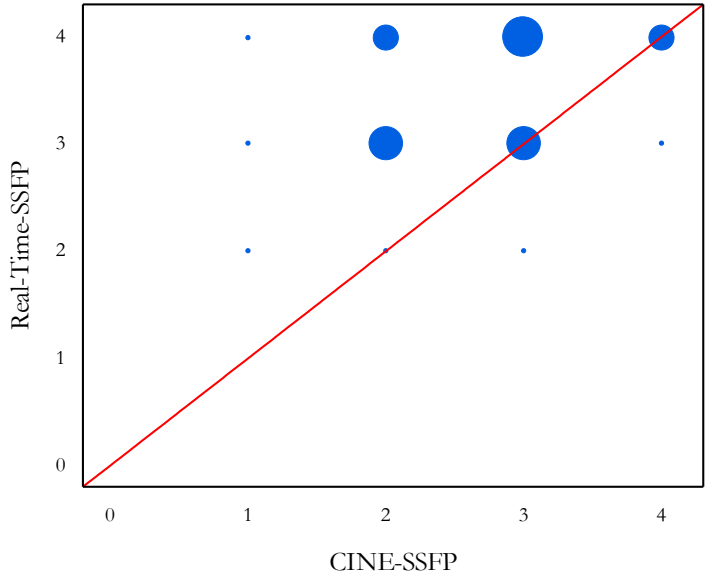

SA apex Gesamteindruck - Untersucher 1

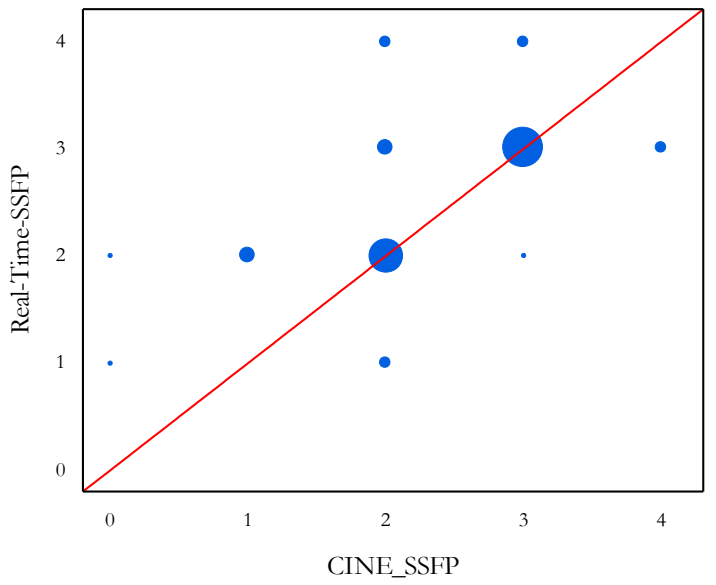

SA basis Gesamteindruck - Untersucher 2

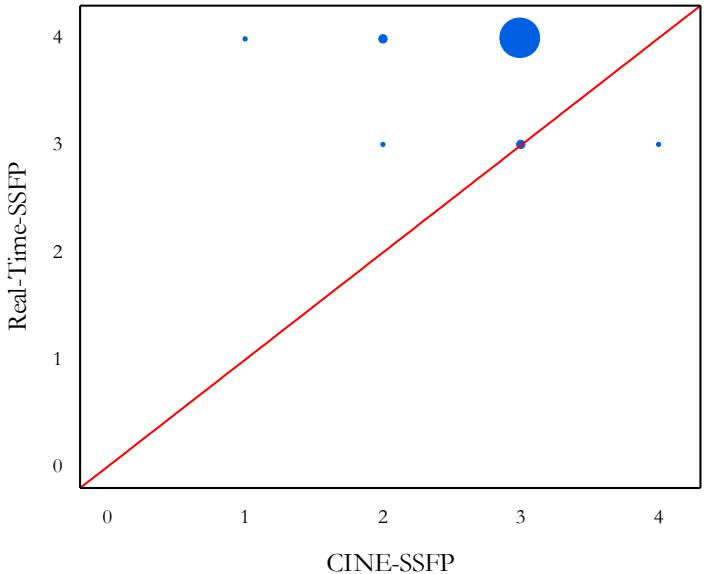

SA mid Gesamteindruck - Unterucher 2

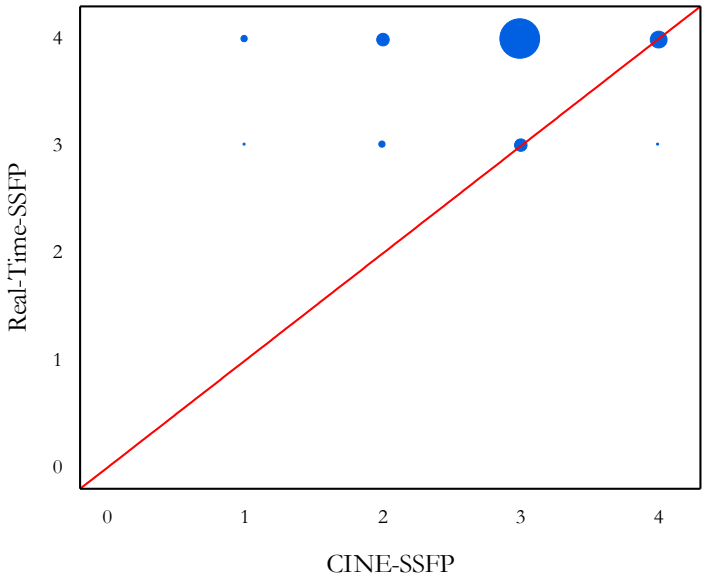

SA apex Gesamteindruck - Untersucher 2

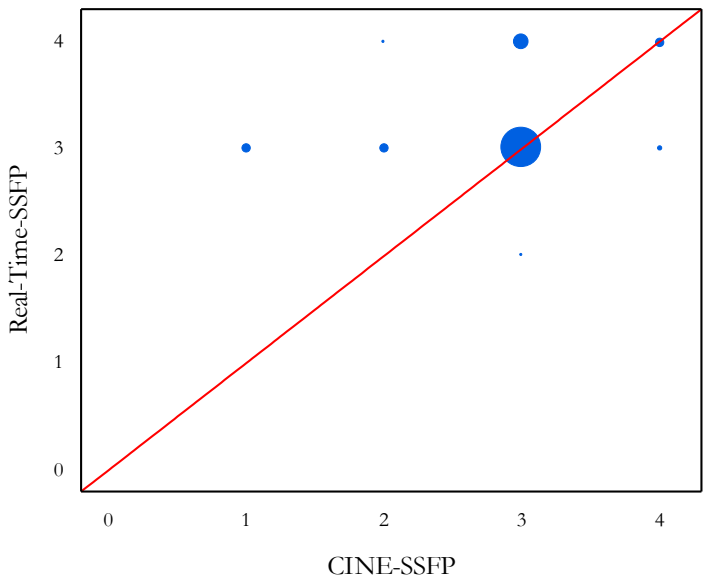

Abbildung 14: Bubbleplots für die Qualitätsanalyse der kurzen Achsen im Gesamteindruck. Die Diagramme zeigen den Vergleich der Bewertungen für CINE-SSFP und Real-Time SSFP für SA basis, mid und apex durch Untersucher 1 (links) und Untersucher 2 (rechts). 

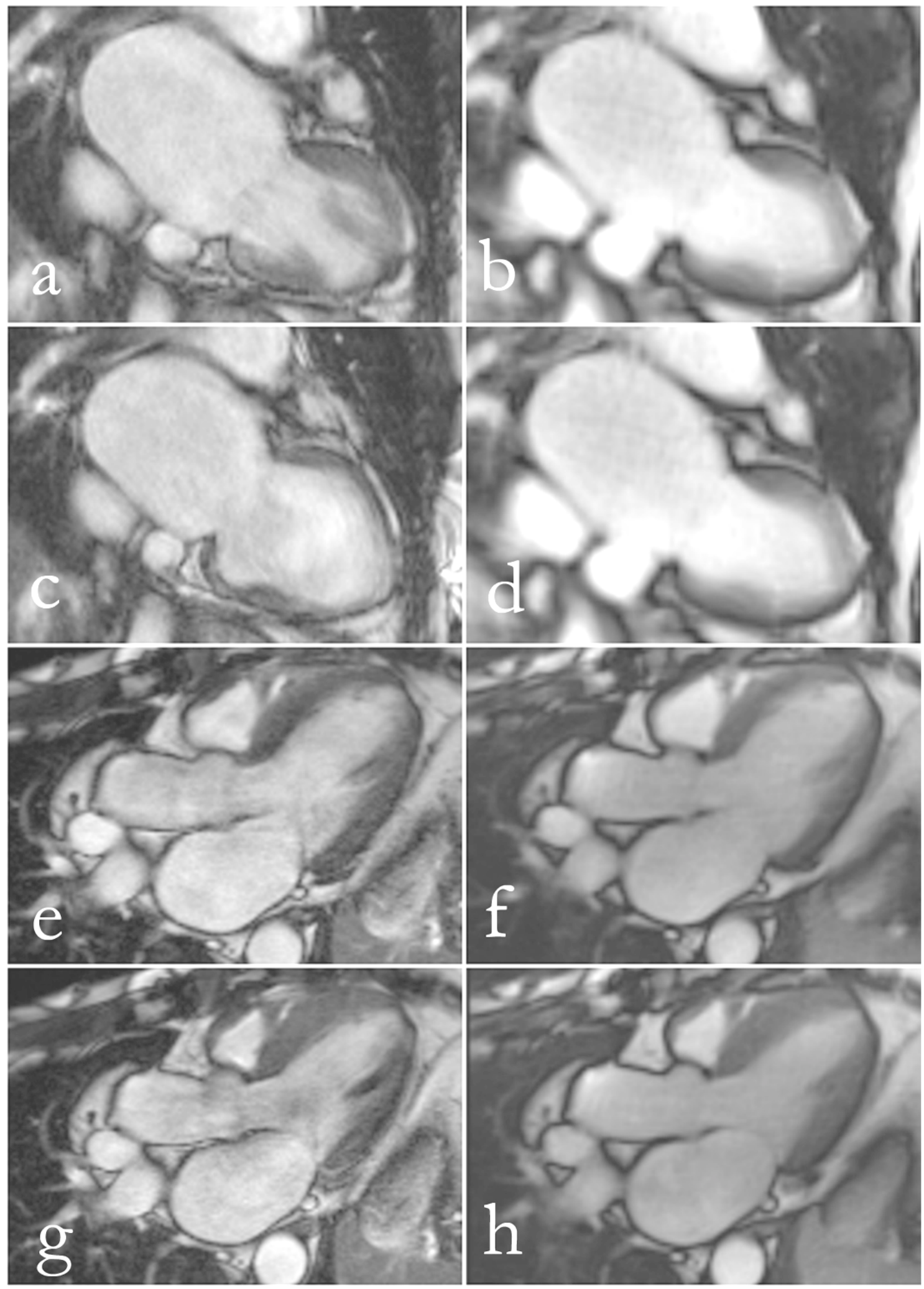

Abbildung 15: Intraindividueller Vergleich CINE- und Real-Time-SSFP-2CV und -3CV.

Oben: Qualität des CINE-SSFP-2CV deutlich eingeschränkt ( $\mathbf{a}=\mathrm{ED}$ und $\mathbf{c}=\mathrm{ES}$ ), Real-TimeSSFP-2CV mit guter Qualität ( $\mathbf{b}=\mathrm{ED}$ und $\mathbf{d}=\mathrm{ES}$ ). Unten: CINE-SSFP-3CV mit reduzierter Diagnosequalität ( $\mathbf{e}=$ ED und $\mathbf{g}=\mathrm{ES}$ ); der Real-Time-SSFP-3CV des gleichen Patienten weist eine bessere Qualität auf $(\mathbf{f}=\mathrm{ED}$ und $\mathbf{h}=\mathrm{ES})$. 

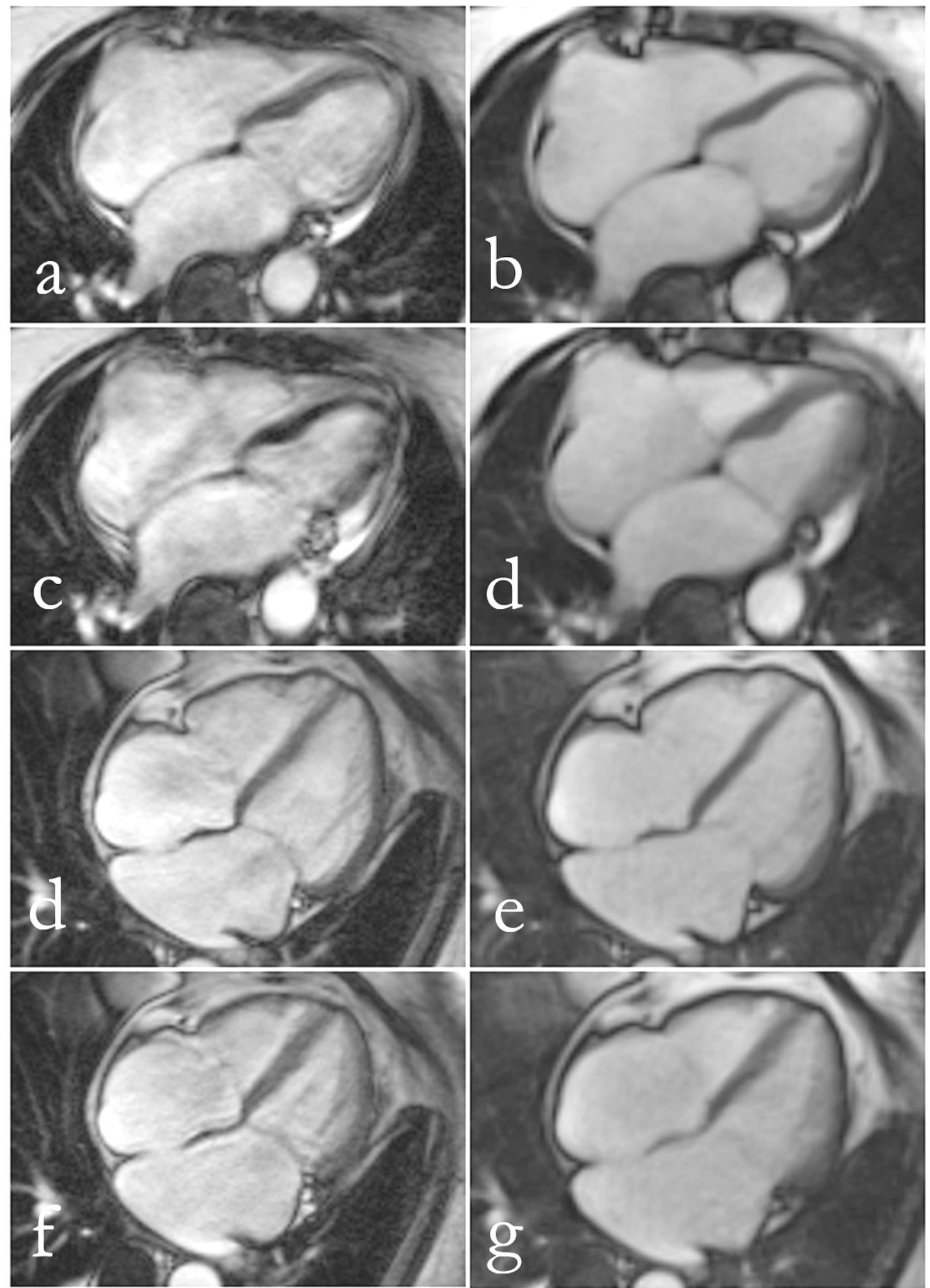

Abbildung 16: Intraindividueller Vergleich CINE- und Real-Time-SSFP-4CV.

Oben: Qualität der CINE-SSFP deutlich eingeschränkt ( $\mathbf{a}=$ ED und $\mathbf{c}=$ ES), Real-Time-SSFP mit guter Qualität ( $\mathbf{b}=$ ED und $\mathbf{d}=$ ES). Unten: CINE- $(\mathbf{e}=$ ED und $\mathbf{g}=$ ES) und Real-Time-SSFP mit guter Qualität $(\mathbf{f}=\mathrm{ED}$ und $\mathbf{h}=\mathrm{ES})$. 

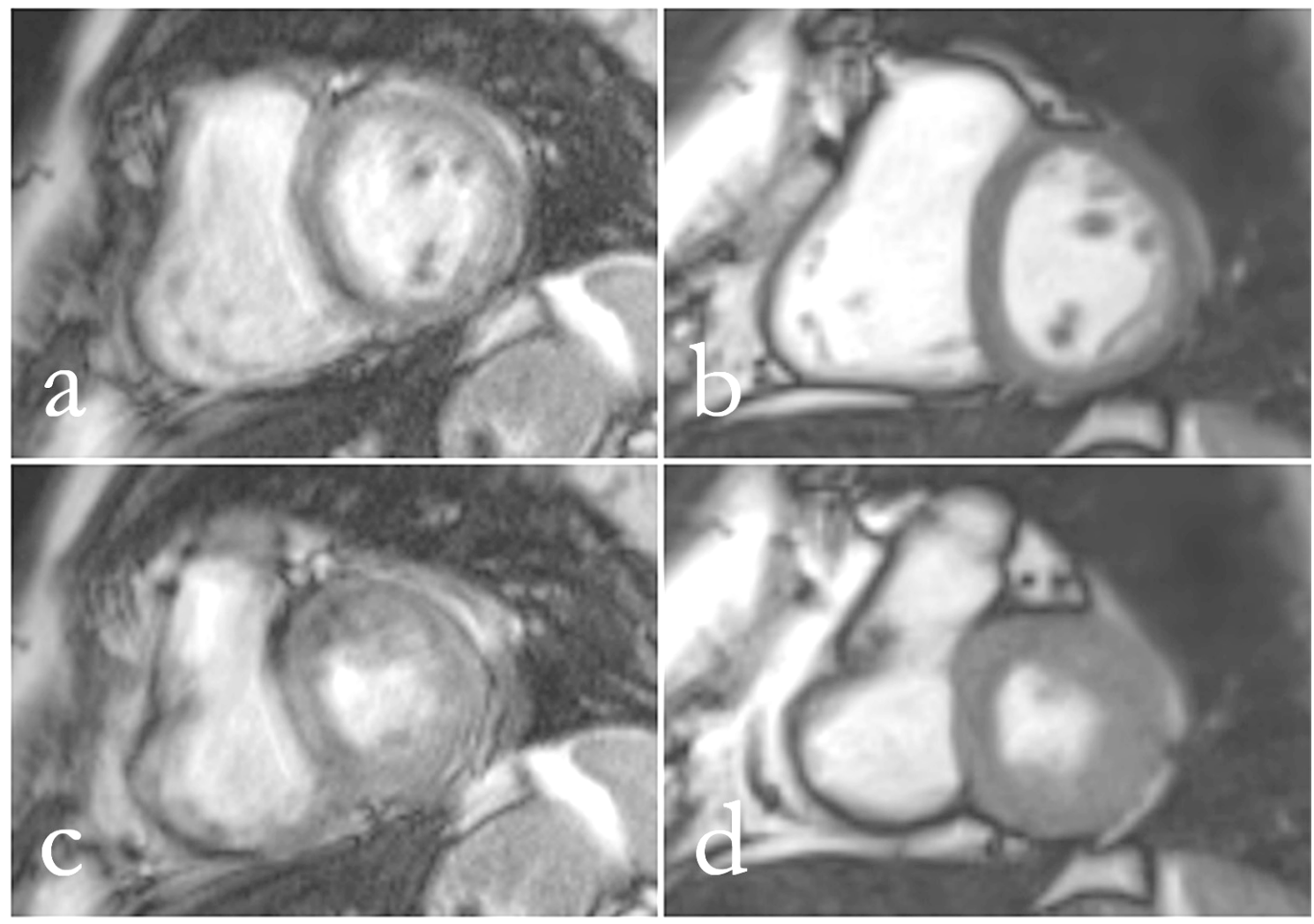

Abbildung 17: Intraindividueller Vergleich CINE- und Real-Time-SSFP-SA-mid. Qualität der CINE-SSFP deutlich eingeschränkt ( $\mathbf{a}=$ ED und $\mathbf{c}=$ ES), Real-Time-SSFP mit guter Qualität ( $\mathbf{b}=$ ED und $\mathbf{d}=$ ES). 


\subsubsection{Abgrenzbarkeit der anterioren und inferioren Wand im 2CV}

Meist wurden CINE-SSFP und Real-Time-SSFP hinsichtlich Abgrenzbarkeit der anterioren und inferioren Wand signifikant unterschiedlich bewertet. Für die anteriore Wand gab es bei Untersucher 1 keinen signifikanten Unterschied. Die zugehörigen $\mathrm{p}$-Werte sind in Tabelle 7 aufgeführt.

Bei Vorliegen eines signifikanten Unterschieds schnitten die Real-Time-SSFP-Aufnahmen besser ab, was nur anhand der Bubble-Plots zu erkennen ist, die deshalb in Abbildung 18 dargestellt sind.

\subsubsection{Abgrenzbarkeit des Septums und der Seitenwand}

Die Bewertung der Abgrenzbarkeit des Septums und der Seitenwand erfolgte in 3CV, 4CV, SA apex, mid und basis. In den meisten Fällen unterschied sich die Bewertung von CINEund Real-Time-SSFP signifikant. Für Septum und Seitenwand in SA apex sowie für die Seitenwand im 3CV war p nur bei Untersucher 2 kleiner als 0,05 (p-Werte: Tabelle 8 und Tabelle 9).

Da sich nur anhand der Bubble-Plots prüfen lässt, welche der beiden Methoden besser bewertet wurde, sind alle zugehörigen Grafiken auf den folgenden Seiten dargestellt (Abbildungen 19 bis 22). Dort ist eine bessere Bewertung der Real-Time-SSFP für alle Schnittebenen abzulesen. Nur die Bubble-Plots für die Bewertung von 3CV (Seitenwand) und SA apex durch Untersucher 1 zeigen eine breite Streuung mit lediglich leichter Tendenz zur gleichen oder besseren Bewertung der Real-Time-SSFP.

\subsubsection{Abgrenzbarkeit der Papillarmuskeln}

Die Sichtbarkeit und Abgrenzbarkeit der Papillarmuskeln wurde nur in SA mid bewertet und unterschied sich nur bei Untersucher 2 signifikant (Tabelle 10).

Die bessere Bewertung der Real-Time-SSFP durch Untersucher 1 ist auf dem zugehörigen Bubble-Plot deutlich erkennbar (Abbildung 23). 
Tabelle 7: p-Werte für Qualitätsanalyse der Abgrenzbarkeit der anterioren und inferioren Wand Ergebnisse des Wilcoxon-matched-pairs-Tests. Signifikante Ergebnisse $(p<0,05)$ sind fett gedruckt.

\begin{tabular}{|c|cc|}
\hline Schnittebene & p Untersucher 1 & $\mathbf{p}$ Untersucher 2 \\
\hline $2 \mathrm{CV}$ - anteriore Wand & 0,1381 & $\mathbf{0 , 0 1 8 4}$ \\
$2 \mathrm{CV}-$ inferiore Wand & $\mathbf{0 , 0 0 7 9}$ & $\mathbf{0 , 0 1 2 0}$ \\
\hline
\end{tabular}

2CV anteriore Wand - Untersucher 1

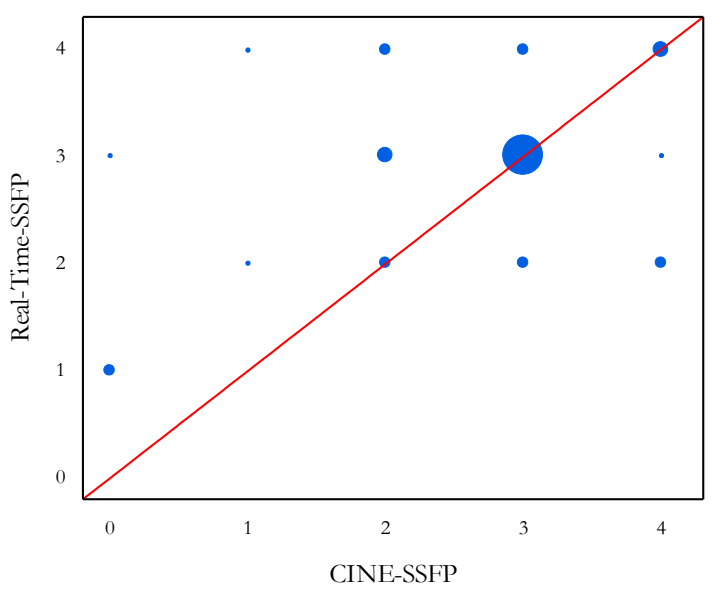

2CV inferore Wand - Untersucher 1

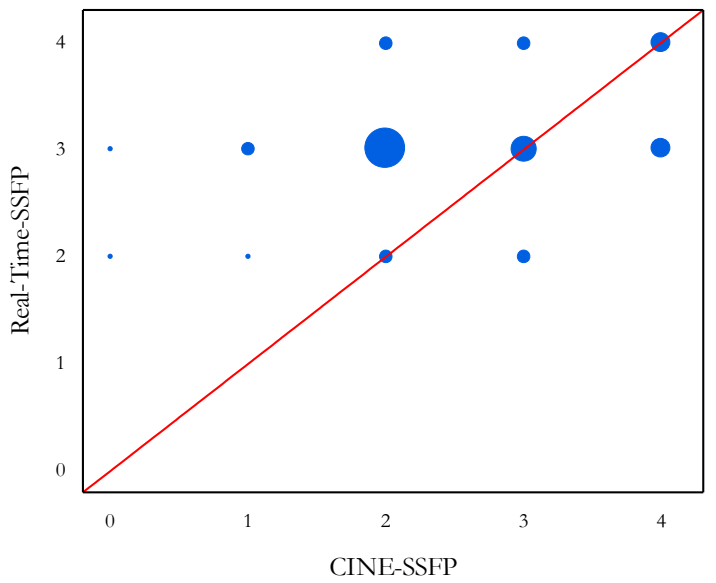

2CV anteriore Wand - Untersucher 2

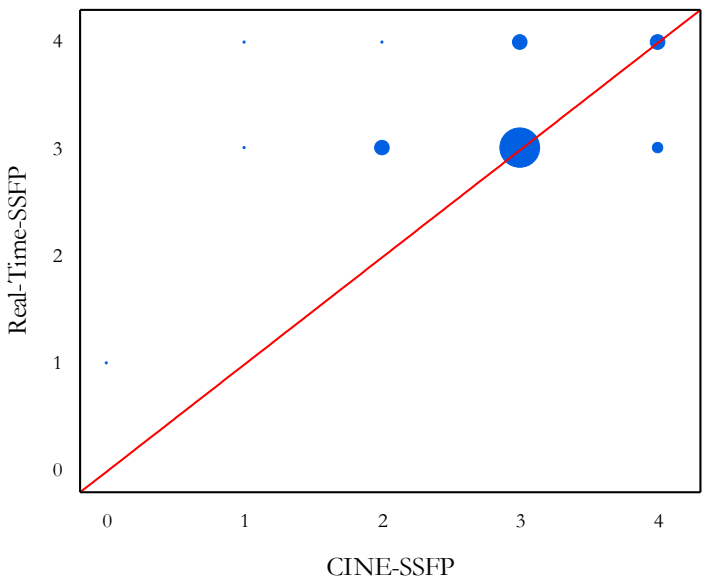

2CV inferiore Wand - Untersucher 2

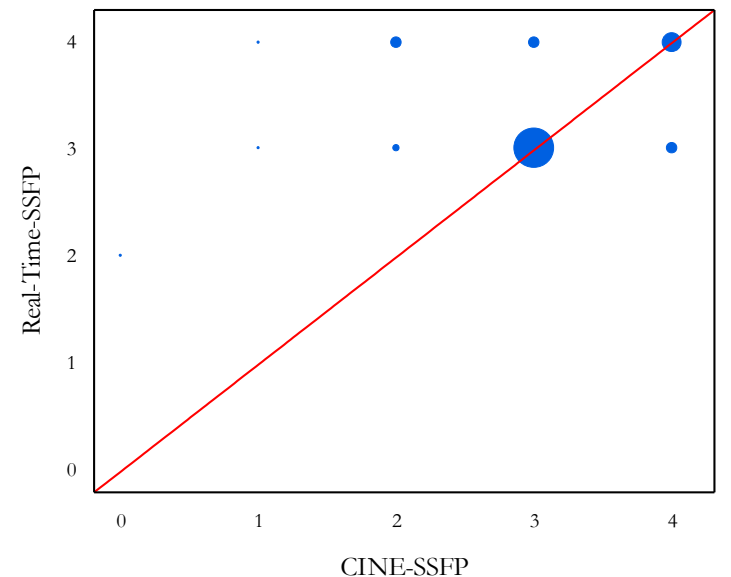

Abbildung 18: Bubble-Plots für Qualitätsanalyse der Abgrenzbarkeit der anterioren und inferioren Wand im $2 \mathrm{CV}$.

Links sind die Bewertungen von Untersucher 1 abgebildet, rechts die von Untersucher 2. 
Tabelle 8: p-Werte für Qualitätsanalyse der Abgrenzbarkeit des Septums - Ergebnisse des Wilcoxon-matched-pairs-Tests. Signifikante Ergebnisse $(p<0,05)$ sind fett gedruckt.

\begin{tabular}{|l|cc|}
\hline Schnittebene & p Untersucher 1 & p Untersucher 2 \\
\hline 3 CV & $\mathbf{0 , 0 3 1 6}$ & $<\mathbf{0 , 0 0 0 1}$ \\
SA basis & $\mathbf{0 , 0 0 3 6}$ & $\mathbf{0 , 0 0 2 6}$ \\
SA mid & $\mathbf{0 , 0 0 0 7}$ & $<\mathbf{0 , 0 0 0 1}$ \\
SA apex & $\mathbf{0 , 0 0 0 7}$ & $\mathbf{0 , 0 0 0 2}$ \\
& 0,1064 & $\mathbf{0 , 0 3 4 3}$ \\
\hline
\end{tabular}
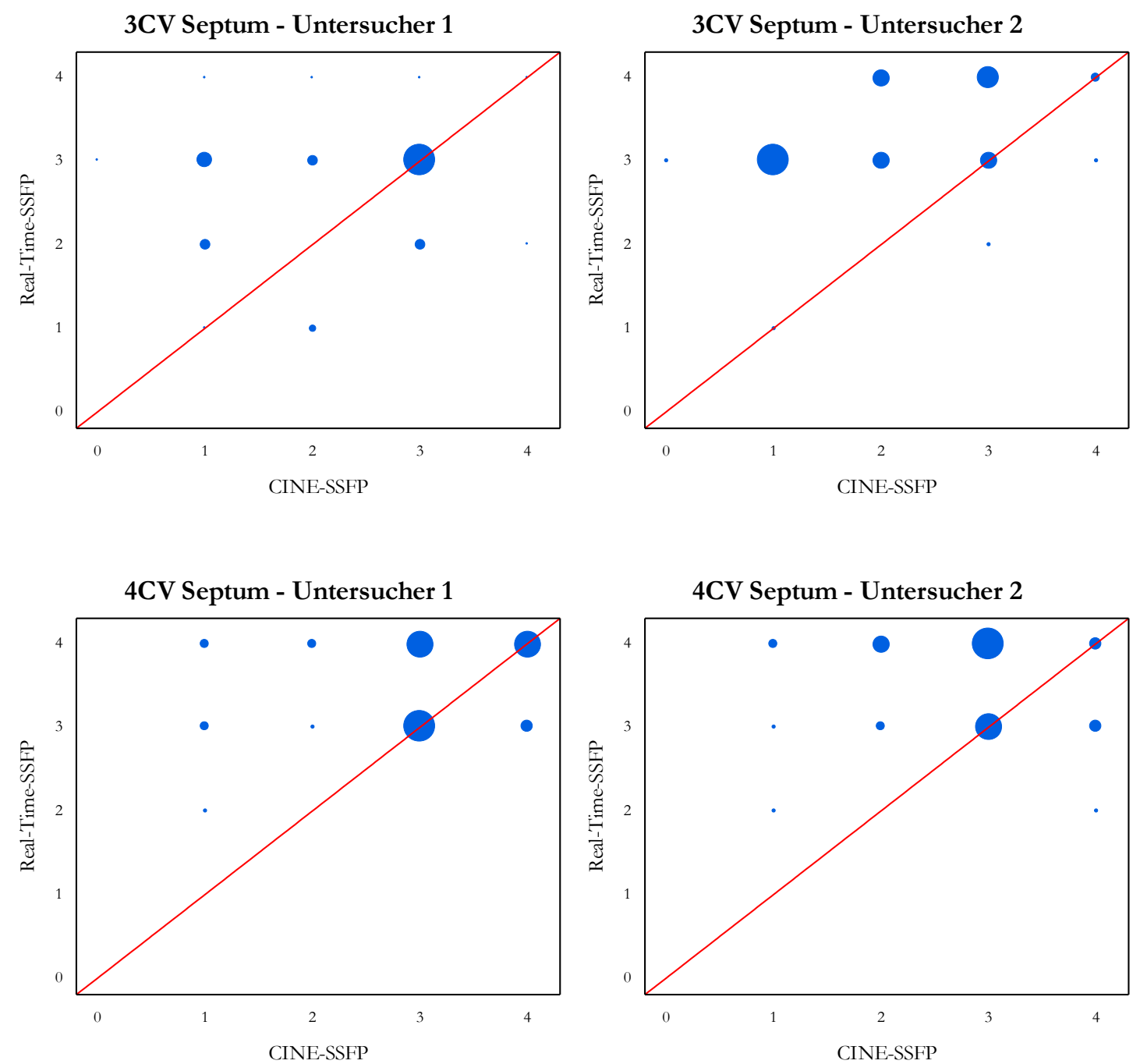

Abbildung 19: Bubble-Plots für die Bewertung des Septums in den langen Achsen.

Vergleich zwischen CINE-SSFP und Real-Time-SSFP für 3CV und 4CV; links: Untersucher 1, rechts: Untersucher 2. 
SA basis Septum - Untersucher 1
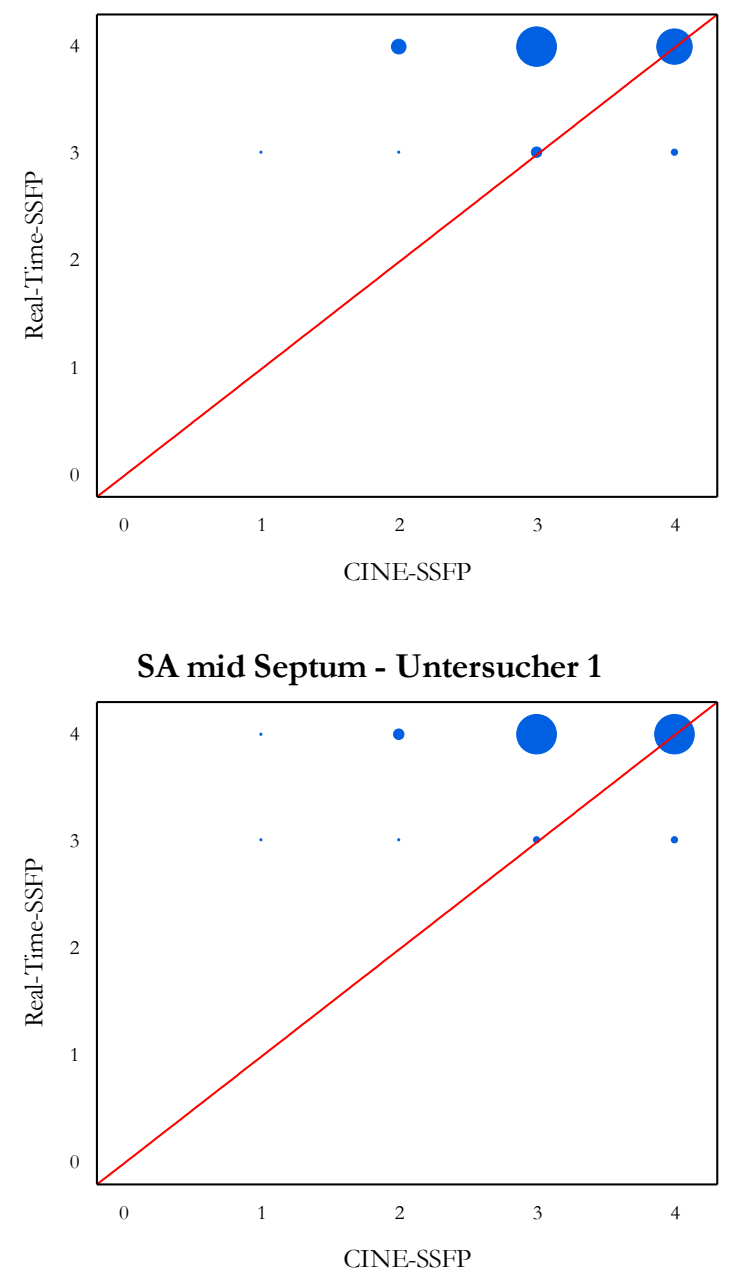

SA apex Septum - Untersucher 1

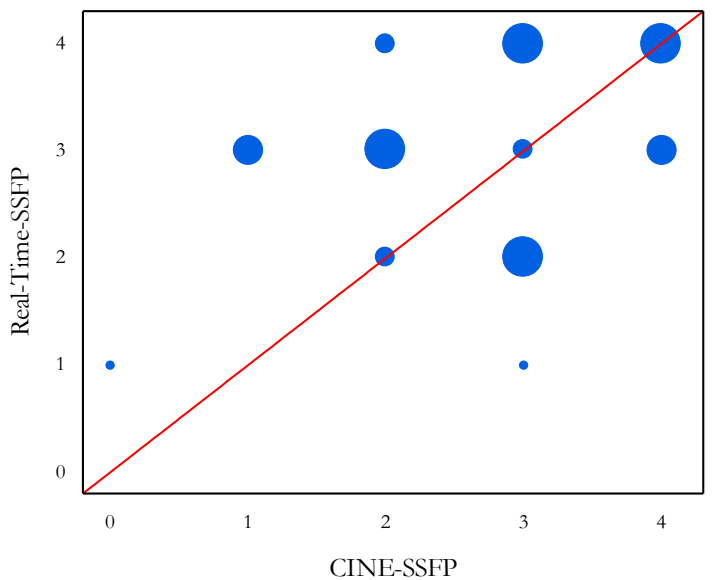

SA basis Septum - Untersucher 2

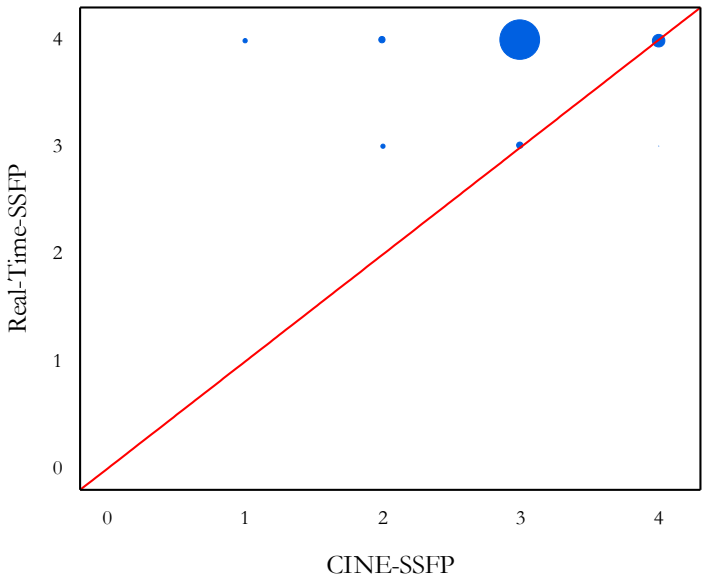

SA mid Septum - Untersucher 2

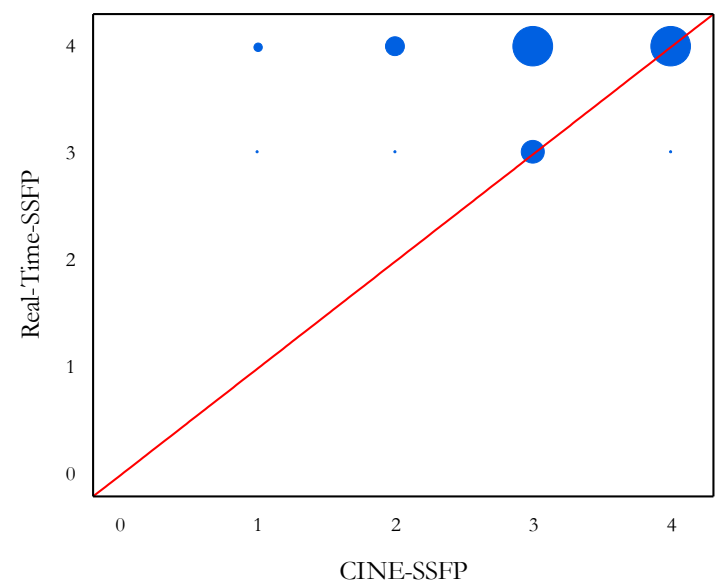

SA apex Septum - Untersucher 2

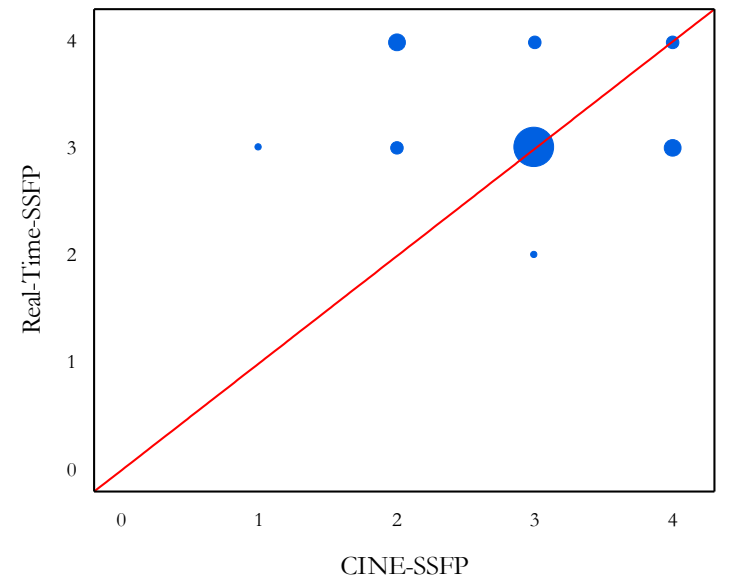

Abbildung 20: Bubble-Plots für die Bewertung des Septums in den kurzen Achsen.

Vergleich zwischen CINE-SSFP und Real-Time-SSFP für SA basis, SA mid und SA apex; links: Untersucher 1, rechts: Untersucher 2. 
Tabelle 9: p-Werte für Qualitätsanalyse der Abgrenzbarkeit der Seitenwand - Ergebnisse des Wilcoxon-matched-pairs-Tests. Signifikante Ergebnisse $(p<0,05)$ sind fett gedruckt.

\begin{tabular}{|l|cc|}
\hline Schnittebene & p Untersucher 1 & p Untersucher 2 \\
\hline 3 CV & 0,1691 & $<\mathbf{0 , 0 0 0 1}$ \\
SA basis & $\mathbf{0 , 0 3 5 1}$ & $\mathbf{0 , 0 0 1 5}$ \\
SA mid & $\mathbf{0 , 0 0 0 6}$ & $<\mathbf{0 , 0 0 0 1}$ \\
SA apex & $\mathbf{0 , 0 1 2 4}$ & $<\mathbf{0 , 0 0 0 1}$ \\
& 0,07076 & $\mathbf{0 , 0 0 7 9}$ \\
\hline
\end{tabular}
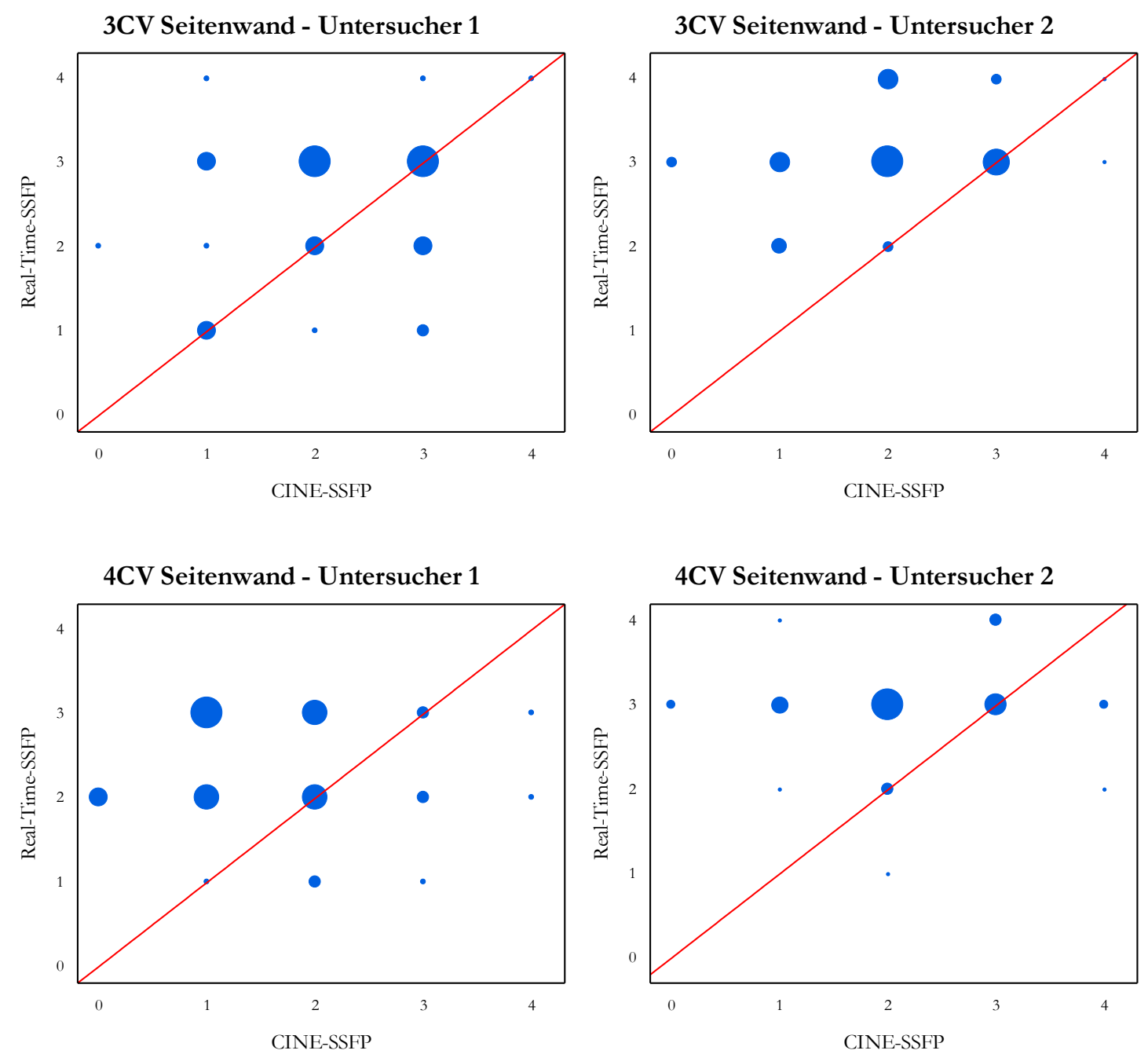

Abbildung 21: Bubble-Plots für die Bewertung der Seitenwand in den langen Achsen.

Vergleich zwischen CINE-SSFP und Real-Time-SSFP für 3CV und 4CV; links: Untersucher 1, rechts: Untersucher 2. 
SA basis Seitenwand - Untersucher 1

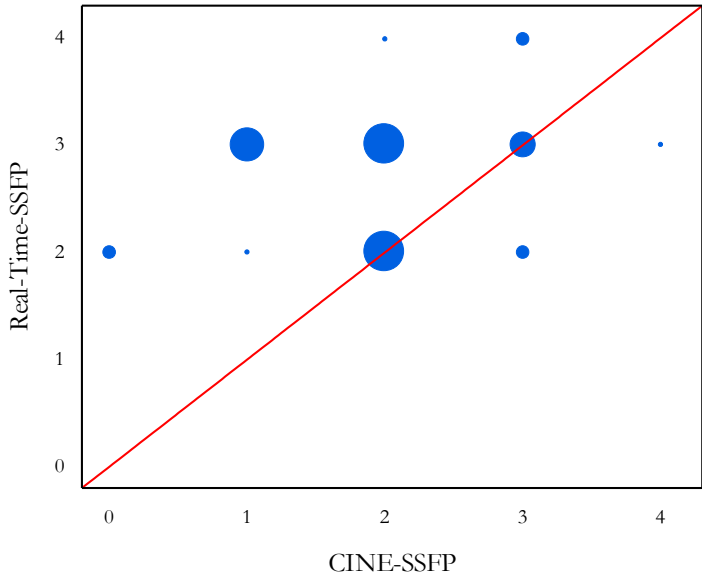

SA mid Seitenwand - Untersucher 1

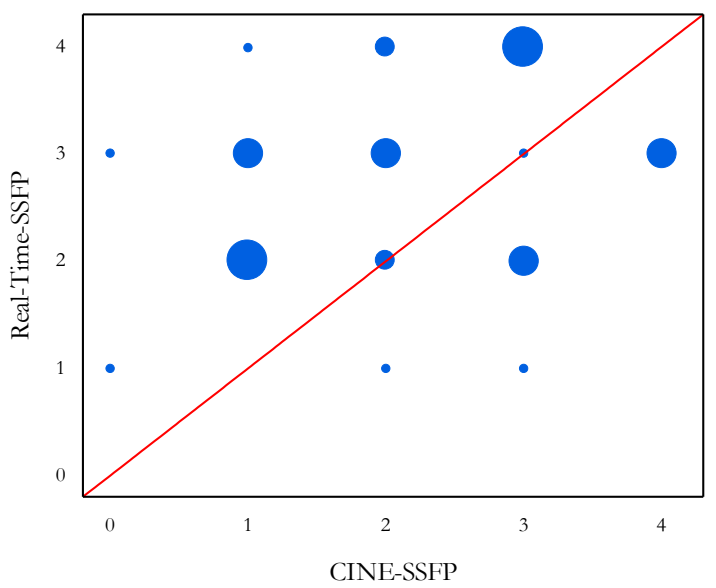

SA apex Seitenwand - Untersucher 1

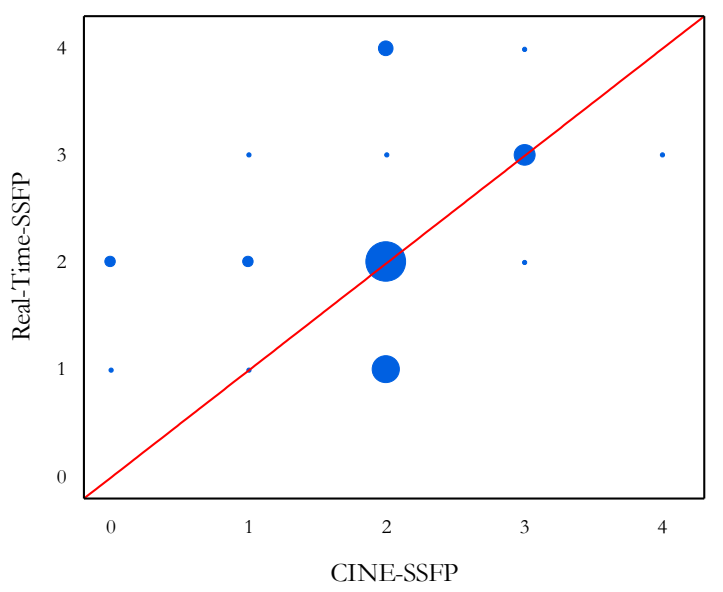

SA basis Seitenwand - Untersucher 2

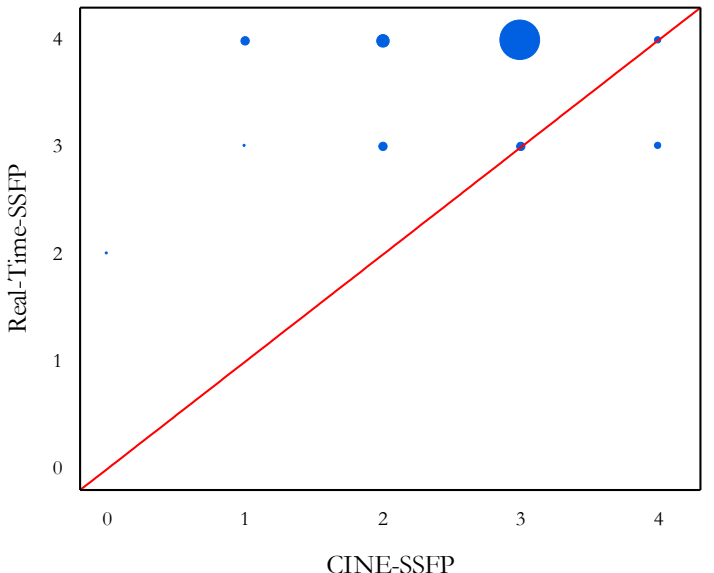

SA mid Seitenwand - Untersucher 2

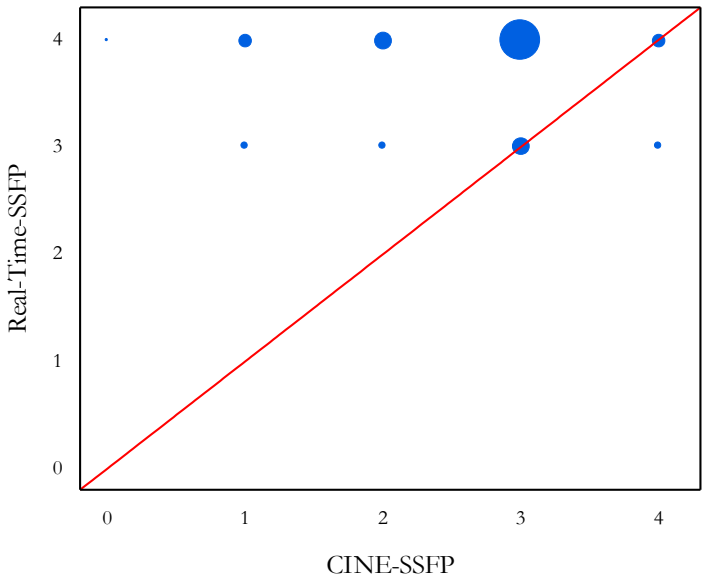

SA apex Seitenwand - Untersucher 2

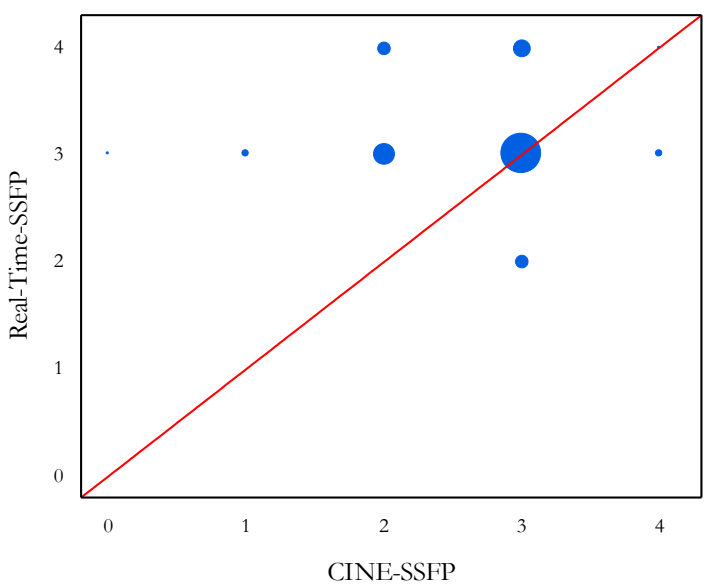

Abbildung 22: Bubble-Plots für die Bewertung der Seitenwand in den kurzen Achsen.

Vergleich zwischen CINE-SSFP und Real-Time-SSFP für SA basis, SA mid und SA apex; links: Untersucher 1, rechts: Untersucher 2. 
Tabelle 10: p-Werte für Qualitätsanalyse der Sichtbarkeit und Abgrenzbarkeit der Papillarmuskeln: Ergebnisse des Wilcoxon-matched-pairs-Tests. Signifikante Ergebnisse $(p<0,05)$ sind fett gedruckt.

\begin{tabular}{|l|cc|}
\hline Schnittebene & p Untersucher 1 & $\mathbf{p}$ Untersucher 2 \\
\hline SA mid & 0,0546 & $\mathbf{0 , 0 0 4 5}$ \\
\hline
\end{tabular}

SA mid Papillarmuskeln - Untersucher 1

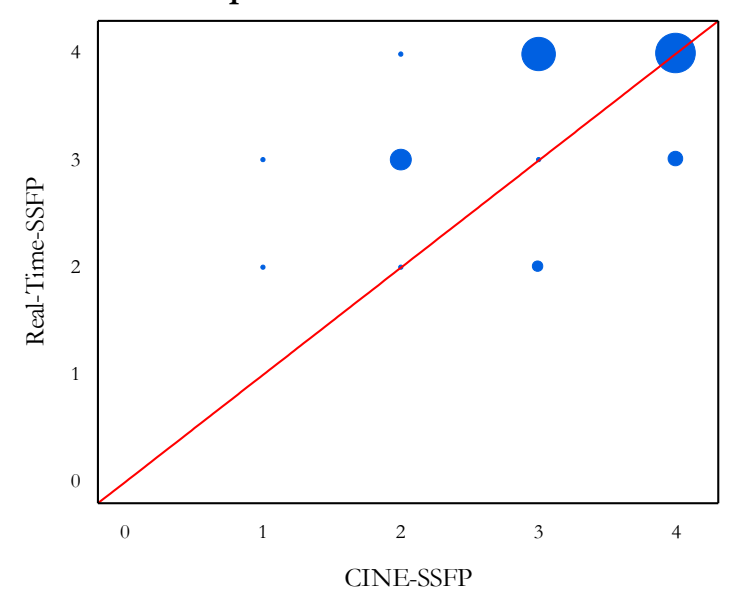

SA mid Papillarmuskeln - Untersucher 2

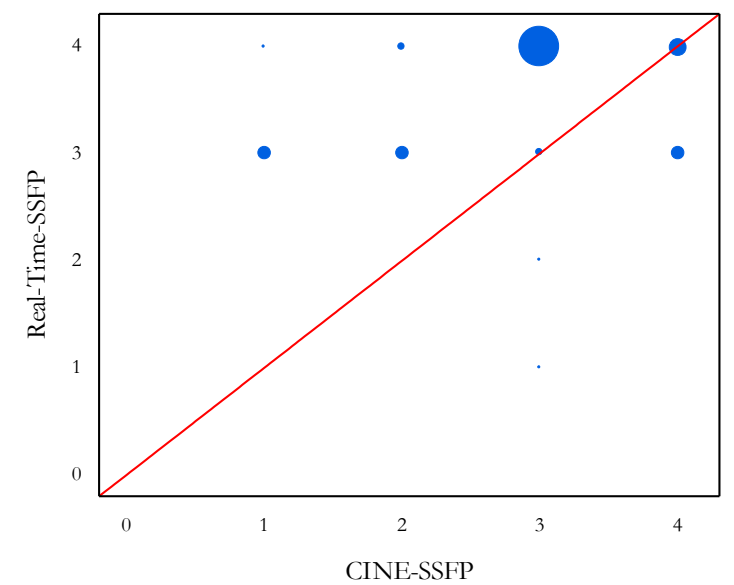

Abbildung 23: Bubble-Plots für die Qualitätsanalyse der Abgrenzbarkeit der Papillarmuskeln.

Vergleich zwischen CINE-SSFP und Real-Time-SSFP für SA mid; links: Untersucher 1, rechts: Untersucher 2.

\subsubsection{Interrater-Variabilität und Intrarater-Variabilität}

Anhand von Bubble-Plots konnte nachvollzogen werden, dass Untersucher 2 im Gesamteindruck häufig bessere Noten gab als Untersucher 1. Eine Signifikanz für diese Tendenz konnte für alle Schnittebenen außer CINE-SSFP-3CV und -4CV nachgewiesen werden.

Auch bei den Einzelkriterien gab Untersucher 2 häufig bessere Noten. Nur die Abgrenzbarkeit des Septums unterschied sich in den meisten Fällen nicht signifikant zwischen den beiden Untersuchern.

Zwischen den beiden Bewertungsdurchgängen unterschieden sich die Noten von Untersucher 1 für die Real-Time-SSFP-Aufnahmen nicht signifikant. Gleiches gilt für die Bewertung der CINE-SSFP mit wenigen Ausnahmen, bei denen im ersten Durchgang bessere Noten vergeben wurden. Bei Untersucher 2 unterschieden sich die Bewertungen weder für CINE- noch für Real-Time-SSFP signifikant zwischen erstem und zweitem Durchgang. 
Aus Gründen der Übersicht sind nicht alle p-Werte und Bubble-Plots für diese Paarvergleiche dargestellt.

\subsection{Linksventrikuläre Funktion}

Die linksventrikuläre Funktion wurde in CINE- und Real-Time-SSFP aus SA-Stapel und 4CV bestimmt. Nicht sinnvoll segmentierbare Bildserien wurden zuvor ausgeschlossen wie in Kapitel 2.5 beschrieben. Bei der Real-Time-SSFP wurden die Ergebnisse nach einer Mittelung über sieben Stapel bzw. Schläge pro Patient zugrundegelegt.

Der CINE-SSFP-SA-Stapel konnte bei 26 von 30 Patienten sinnvoll segmentiert werden. Tabelle 11 zeigt die Werte für die mit dieser Methode erhobenen funktionellen Parameter.

Tabelle 11: Funktionelle Parameter - CINE-SSFP-SA-Stapel; $\mathrm{n}=26$ von 30.

\begin{tabular}{|l|ccccc|}
\hline Parameter & Mittelwert & Median & Minimum & Maximum & Stabw. \\
\hline MM (g) & 120 & 114 & 62 & 219 & 43 \\
EDV $(\mathrm{ml})$ & 123 & 114 & 76 & 304 & 46 \\
ESV $(\mathrm{ml})$ & 69 & 60 & 30 & 237 & 43 \\
SV $(\mathrm{ml})$ & 54 & 53 & 29 & 98 & 14 \\
EF $(\%)$ & 47 & 45 & 22 & 66 & 13 \\
HZV $(1 / \mathrm{min})$ & 4,5 & 4,4 & 2,4 & 8,0 & 1,3 \\
HF $($ Schläge $/ \mathrm{min})$ & 85 & 83 & 56 & 138 & 22 \\
& & & & & 19 \\
MM-Index $\left(\mathrm{g} / \mathrm{m}^{2}\right)$ & 61 & 56 & 39 & 110 & 18 \\
EDV-Index $\left(\mathrm{ml} / \mathrm{m}^{2}\right)$ & 62 & 57 & 43 & 139 & 19 \\
ESV-Index $\left(\mathrm{ml} / \mathrm{m}^{2}\right)$ & 35 & 30 & 16 & 108 & 19 \\
SV-Index $\left(\mathrm{ml} / \mathrm{m}^{2}\right)$ & 28 & 26 & 18 & 56 & 8,6 \\
HZV-Index $\left(1 / \mathrm{min}^{2} / \mathrm{m}^{2}\right)$ & 2,3 & 2,3 & 1,1 & 4,6 & \\
\hline
\end{tabular}


Im CINE-SSFP-4CV waren 24 von 30 Untersuchungen auswertbar. Tabelle 12 zeigt die ermittelten Werte.

Tabelle 12: Funktionelle Parameter - CINE-SSFP-4CV; $\mathrm{n}=24$ von 30.

\begin{tabular}{|c|c|c|c|c|c|}
\hline Parameter & Mittelwert & Median & Minimum & Maximum & Stabw. \\
\hline MM (g) & 154 & 141 & 74 & 287 & 58 \\
\hline $\mathrm{EDV}(\mathrm{ml})$ & 140 & 136 & 78 & 281 & 52 \\
\hline ESV (ml) & 65 & 53 & 17 & 201 & 44 \\
\hline SV (ml) & 75 & 74 & 46 & 124 & 20 \\
\hline $\mathrm{EF}(\%)$ & 56 & 58 & 28 & 81 & 14 \\
\hline $\mathrm{HZV}(1 / \mathrm{min})$ & 6,4 & 5,8 & 4,1 & 12,0 & 2,1 \\
\hline HF (Schläge/min) & 87 & 84 & 49 & 145 & 22 \\
\hline MM-Index $\left(\mathrm{g} / \mathrm{m}^{2}\right)$ & 77 & 68 & 39 & 139 & 27 \\
\hline EDV-Index $\left(\mathrm{ml} / \mathrm{m}^{2}\right)$ & 70 & 64 & 41 & 128 & 22 \\
\hline ESV-Index $\left(\mathrm{ml} / \mathrm{m}^{2}\right)$ & 32 & 25 & 9 & 91 & 20 \\
\hline SV-Index $\left(\mathrm{ml} / \mathrm{m}^{2}\right)$ & 38 & 37 & 24 & 59 & 10 \\
\hline $\begin{array}{l}\text { HZV-Index } \\
\left(1 / \mathrm{min} / \mathrm{m}^{2}\right)\end{array}$ & 3,3 & 3,0 & 2,1 & 5,6 & 1,0 \\
\hline
\end{tabular}


Der Real-Time-SSFP-SA-Stapel konnte bei 29 von 30 Patienten segmentiert werden. Bei einem Patienten war eine Segmentation aufgrund technischer Probleme bei der Bildsortierung, die im Anschluss behoben wurden, nicht möglich. Die Ergebnisse der Segmentation zeigt Tabelle 13.

Tabelle 13: Funktionelle Parameter - Real-Time-SSFP-SA-Stapel; $n=29$ von 30.

\begin{tabular}{|c|c|c|c|c|c|}
\hline Parameter & Mittelwert & Median & Minimum & Maximum & Stabw. \\
\hline MM (g) & 108 & 98 & 56 & 187 & 39 \\
\hline $\mathrm{EDV}(\mathrm{ml})$ & 112 & 103 & 71 & 291 & 43 \\
\hline ESV (ml) & 60 & 51 & 21 & 222 & 39 \\
\hline $\mathrm{SV}(\mathrm{ml})$ & 52 & 51 & 32 & 82 & 13 \\
\hline $\mathrm{EF}(\%)$ & 50 & 52 & 23 & 78 & 13 \\
\hline $\mathrm{HZV}(1 / \mathrm{min})$ & 4,0 & 4,0 & 2,7 & 5,6 & 0,8 \\
\hline HF (Schläge/min) & 80 & 78 & 52 & 127 & 167 \\
\hline MM-Index $\left(\mathrm{g} / \mathrm{m}^{2}\right)$ & 55 & 49 & 33 & 110 & 18 \\
\hline EDV-Index $\left(\mathrm{ml} / \mathrm{m}^{2}\right)$ & 57 & 52 & 38 & 133 & 18 \\
\hline ESV-Index $\left(\mathrm{ml} / \mathrm{m}^{2}\right)$ & 30 & 24 & 14 & 101 & 18 \\
\hline SV-Index $\left(\mathrm{ml} / \mathrm{m}^{2}\right)$ & 27 & 27 & 15 & 49 & 7 \\
\hline $\begin{array}{l}\text { HZV-Index } \\
\left(1 / \mathrm{min} / \mathrm{m}^{2}\right)\end{array}$ & 2,1 & 2 & 1,2 & 3,0 & 0,4 \\
\hline
\end{tabular}


Im Real-Time-SSFP-4CV wurde ein Patient in der Qualitätsanalyse so schlecht bewertet, dass er aus der funktionellen Auswertung herausgenommen wurde. Alle übrigen Aufnahmen konnten segmentiert werden. Eine Zusammenfassung der Werte ist Tabelle $14 \mathrm{zu}$ entnehmen.

Tabelle 14: Funktionelle Parameter -Real-Time-SSFP-4CV; $\mathrm{n}=29$ von 30.

\begin{tabular}{|c|c|c|c|c|c|}
\hline Parameter & Mittelwert & Median & Minimum & Maximum & Stabw. \\
\hline MM (g) & 141 & 138 & 62 & 265 & 49 \\
\hline $\mathrm{EDV}(\mathrm{ml})$ & 127 & 123 & 63 & 280 & 49 \\
\hline ESV (ml) & 62 & 56 & 13 & 209 & 42 \\
\hline $\mathrm{SV}(\mathrm{ml})$ & 64 & 65 & 31 & 118 & 19 \\
\hline $\mathrm{EF}(\%)$ & 53 & 53 & 25 & 82 & 14 \\
\hline $\mathrm{HZV}(1 / \mathrm{min})$ & 5,1 & 5,2 & 2,7 & 8,0 & 1,3 \\
\hline HF (Schläge/min) & 83 & 79 & 51 & 144 & 20 \\
\hline MM-Index $\left(\mathrm{g} / \mathrm{m}^{2}\right)$ & 71 & 69 & 39 & 122 & 21 \\
\hline EDV-Index $\left(\mathrm{ml} / \mathrm{m}^{2}\right)$ & 64 & 59 & 33 & 127 & 21 \\
\hline ESV-Index $\left(\mathrm{ml} / \mathrm{m}^{2}\right)$ & 31 & 28 & 7 & 95 & 19 \\
\hline SV-Index $\left(\mathrm{ml} / \mathrm{m}^{2}\right)$ & 33 & 33 & 16 & 57 & 9 \\
\hline $\begin{array}{l}\text { HZV-Index } \\
\left(1 / \mathrm{min} / \mathrm{m}^{2}\right)\end{array}$ & 2,6 & 2,5 & 1,5 & 3,9 & 0,6 \\
\hline
\end{tabular}


Bei 28 von 30 Patienten lagen auswertbare Echokardiographien vor. Die Analyse mit der biplanen Scheibchensummationsmethode nach Simpson über fünf Schläge ergab folgende Ergebnisse (Tabelle 15):

Tabelle 15: Funktionelle Parameter - Echokardiographie; $\mathrm{n}=28$ von 30.

\begin{tabular}{|l|ccccc|}
\hline \multicolumn{1}{|c|}{ Parameter } & Mittelwert & Median & Minimum & Maximum & Stabw. \\
\hline EDV (ml) & 120 & 105 & 68 & 281 & 48 \\
ESV (ml) & 64 & 53 & 38 & 226 & 38 \\
SV (ml) & 56 & 54 & 22 & 106 & 20 \\
EF (\%) & 48 & 51 & 20 & 63 & 10 \\
HZV $(1 / \mathrm{min})$ & 4,9 & 4,4 & 2,5 & 8,9 & 1,8 \\
& & & & & \\
EDV-Index $\left(\mathrm{ml} / \mathrm{m}^{2}\right)$ & 60 & 55 & 36 & 128 & 19 \\
ESV-Index $\left(\mathrm{ml} / \mathrm{m}^{2}\right)$ & 32 & 27 & 19 & 103 & 16 \\
SV-Index $\left(\mathrm{ml} / \mathrm{m}^{2}\right)$ & 28 & 27 & 10 & 49 & 9 \\
$\begin{array}{l}\text { HZV-Index } \\
\left(\mathrm{l} / \mathrm{min} / \mathrm{m}^{2}\right)\end{array}$ & 2,4 & 2,4 & 1,2 & 4,1 & 0,7 \\
\hline
\end{tabular}

\subsubsection{Vergleich der linksventrikulären Funktionsparameter in CINE-SSFP und Real-Time-SSFP}

Für die funktionellen Parameter fand ein Vergleich zwischen den mit QMass erhobenen Werten aus CINE-SSFP und Real-Time-SSFP für den SA-Stapel und den 4CV statt. Bei der Real-Time-SSFP wurden die Ergebnisse der Mittelung über sieben Stapel bzw. Schläge verwendet.

Für den Vergleich der Ergebnisse aus dem SA-Stapel konnten 25 von 30 Untersuchungen verwendet werden, da vier Untersuchungen in der CINE-SSFP und eine in Real-TimeSSFP nicht sinnvoll ausgewertet werden konnten. Die Band-Altman-Analyse, bei der die Werte der CINE-SSFP von denen der Real-Time-SSFP abgezogen wurden, ergab folgendes:

Mit der Real-Time-SSFP wurden im Mittel etwas niedrigere Werte gemessen als mit der CINE-SSFP. Bei einer Verzerrung von $2 \%$ (Übereinstimmungsgrenzen: -15\% und $19 \%$ ) wurde lediglich die EF durch die Real-Time-SSFP leicht überschätzt. Tabelle 16 zeigt die 
Ergebnisse der Bland-Altman-Analyse, die in Abbildung 24 für EDV und EF graphisch dargestellt sind.

Tabelle 16: Bland-Altman-Analyse - Vergleich Real-Time-SSFP- vs. CINE-SSFP- SA-Stapel (RealTime - CINE); $\mathrm{n}=25$ von 30; ÜGr. = Übereinstimmungsgrenzen.

\begin{tabular}{|l|ccc|}
\hline \multicolumn{1}{|c|}{ Parameter } & Verzerrung & \multicolumn{3}{|c|}{ ÜGr. } \\
\hline MM (g) & -11 & -45 & 22 \\
EDV (ml) & -8 & -32 & 17 \\
ESV (ml) & -6 & -27 & 14 \\
SV (ml) & -1 & -26 & 25 \\
EF (\%) & 2 & -15 & 19 \\
HZV (l/min) & $-0,4$ & $-2,4$ & 1,5 \\
\hline
\end{tabular}
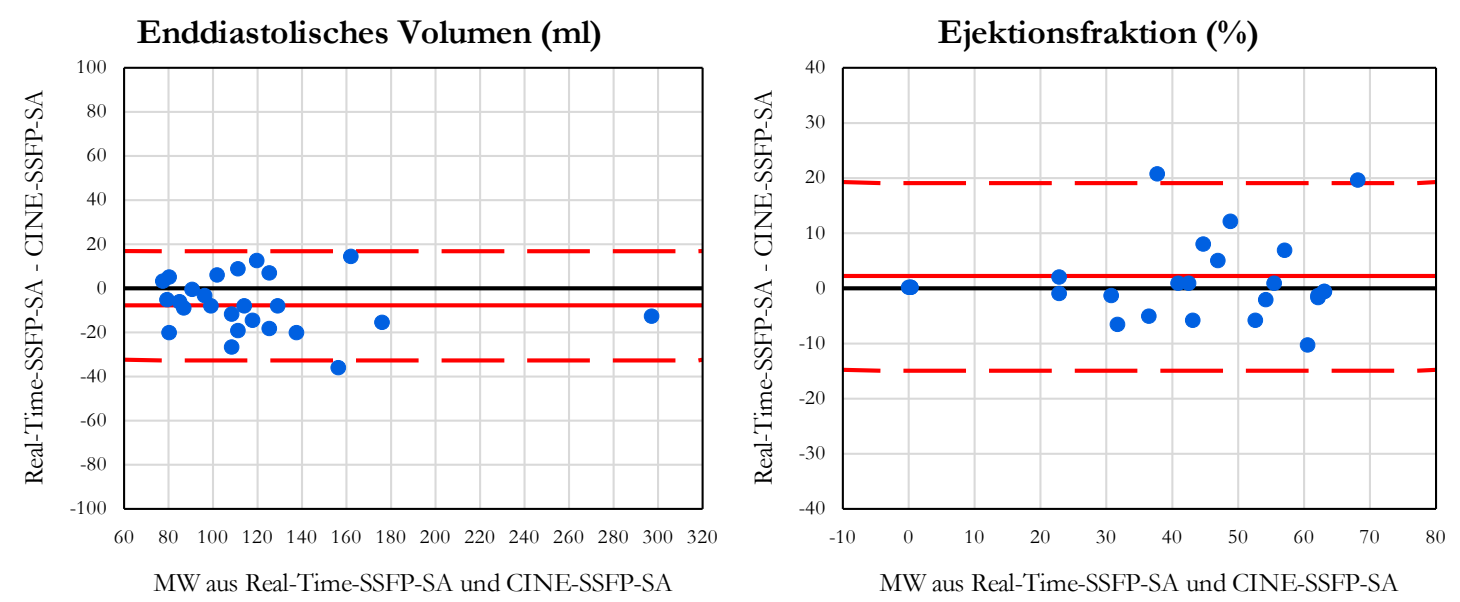

Abbildung 24: Bland-Altman-Plots zum Vergleich zwischen CINE- und Real-Time-SSFP-SAStapel für EDV und EF; MW = Mittelwert. 
Im 4CV konnten 24 Untersuchungen verglichen werden. Fünf Untersuchungen gingen wegen zu schlechter CINE-SSFP-Aufnahmen nicht in die Analyse ein, eine weil CINE und Real-Time gleichermaßen schlechte Qualität zeigten und nicht zuverlässig segmentiert werden konnten. Alle Parameter wurden durch die Real-Time-SSFP im Mittel leicht unterschätzt. Tabelle 17 zeigt die Ergebnisse der Bland-Altman-Analyse, die für EDV und EF in Abbildung 25 graphisch dargestellt sind.

Tabelle 17: Bland-Altman-Analyse: Vergleich Real-Time-SSFP- vs. CINE-SSFP-4CV (Real-Time CINE); $\mathrm{n}=24$ von 30 .

\begin{tabular}{|l|ccc|}
\hline \multicolumn{1}{|c|}{ Parameter } & Verzerrung & ÜGr. \\
\hline MM (g) & -11 & -49 & 26 \\
EDV (ml) & -10 & -38 & 18 \\
ESV (ml) & -1 & -19 & 17 \\
SV (ml) & -9 & -33 & 16 \\
EF (\%) & -3 & -16 & 9 \\
HZV (l/min) & $-1,2$ & $-4,1$ & 1,7 \\
\hline
\end{tabular}
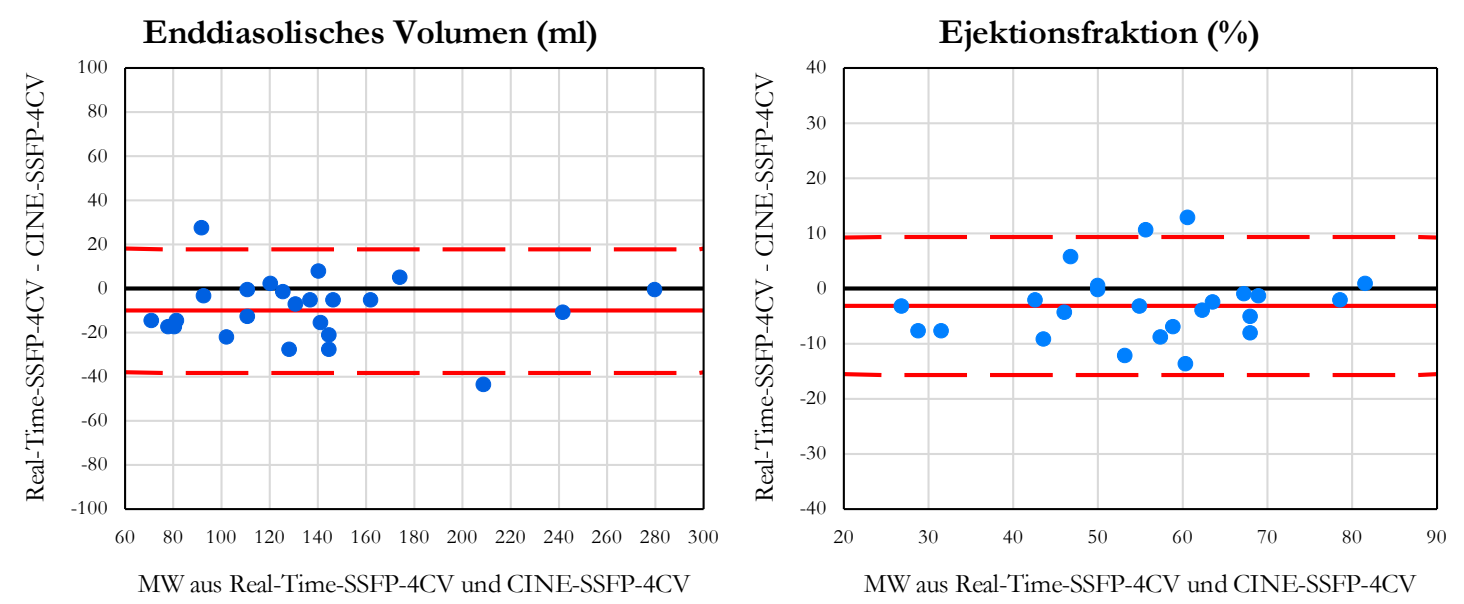

Abbildung 25: Bland-Altman-Plots zum Vergleich zwischen CINE- und Real-Time-SSFP-4CV für EDV und EF. 


\subsubsection{Vergleich der linksventrikulären Funktionsparameter in CINE-SSFP, Real- Time-SSFP und Echokardiographie}

Die Ergebnisse der Auswertung der linksventrikulären Funktion aus der Echokardiographie wurden mit den Ergebnissen der CINE- und Real-Time-SSFP jeweils aus dem 4CV verglichen. Die Zahl der in den Vergleich eingeschlossenen Untersuchungen betrug $n=23$ für den Vergleich Echokardiographie vs. CINE-SSFP-4CV und $n=27$ für den Vergleich Echokardiographie vs. Real-Time-SSFP-4CV.

Die Ergebnisse aus der Echokardiographie wurden in der Bland-Altmann-Analyse jeweils von den Ergebnissen aus der MRT abgezogen. Da die MM nur in der MRT bestimmt wurde, wurde sie bei diesem Vergleich nicht berücksichtigt.

Insgesamt zeigte sich eine Tendenz zur Überschätzung der Werte für die funktionellen Parameter durch die MRT im Vergleich zur Echokardiographie. Nur das ESV wurde durch beide MRT-Modalitäten um wenige $\mathrm{ml}$ unterschätzt. Im Falle einer Überschätzung im Vergleich zur Echokardiographie zeigte sich diese deutlicher in der CINE-SSFP als in der Real-Time-SSFP. Die Werte der Real-Time-SSFP waren also insgesamt denen der Echokardiographie etwas näher. Auffällig ist für beide Vergleiche die breite Streuung. Ergebnisse der Bland-Altman-Analysen sind in Tabelle 18 gegenübergestellt; Abbildung 26 zeigt die zugehörigen Plots für EDV und EF.

Tabelle 18: Bland-Altman-Analyse - CINE-SSFP-4CV vs. Echokardiographie ( $\mathrm{n}=23$ von 30$)$ und Real-Time-SSFP-4CV vs. Echo ( $\mathrm{n}=27$ von 30); MRT - Echo.

\begin{tabular}{|l|ccc|ccc|}
\hline \multirow{2}{*}{ Parameter } & \multicolumn{2}{|c|}{ CINE - Echokardiographie } & \multicolumn{2}{|l|}{ Real-Time - Echokardiographie } \\
& $\begin{array}{l}\text { Ver- } \\
\text { zerrung }\end{array}$ & ÜGr. & $\begin{array}{c}\text { Ver- } \\
\text { zerrung }\end{array}$ & ÜGr. \\
\hline EDV $(\mathrm{ml})$ & 12 & -50 & 74 & 4 & -53 & 61 \\
ESV (ml) & -4 & -49 & 40 & -4 & -42 & 34 \\
SV (ml) & 16 & -23 & 55 & 8 & -27 & 42 \\
EF $(\%)$ & 9 & 13 & 31 & 5 & -16 & 27 \\
HZV $(1 / \mathrm{min})$ & 1,2 & $-2,3$ & 4,7 & 0,1 & $-2,8$ & 2,9 \\
\hline
\end{tabular}



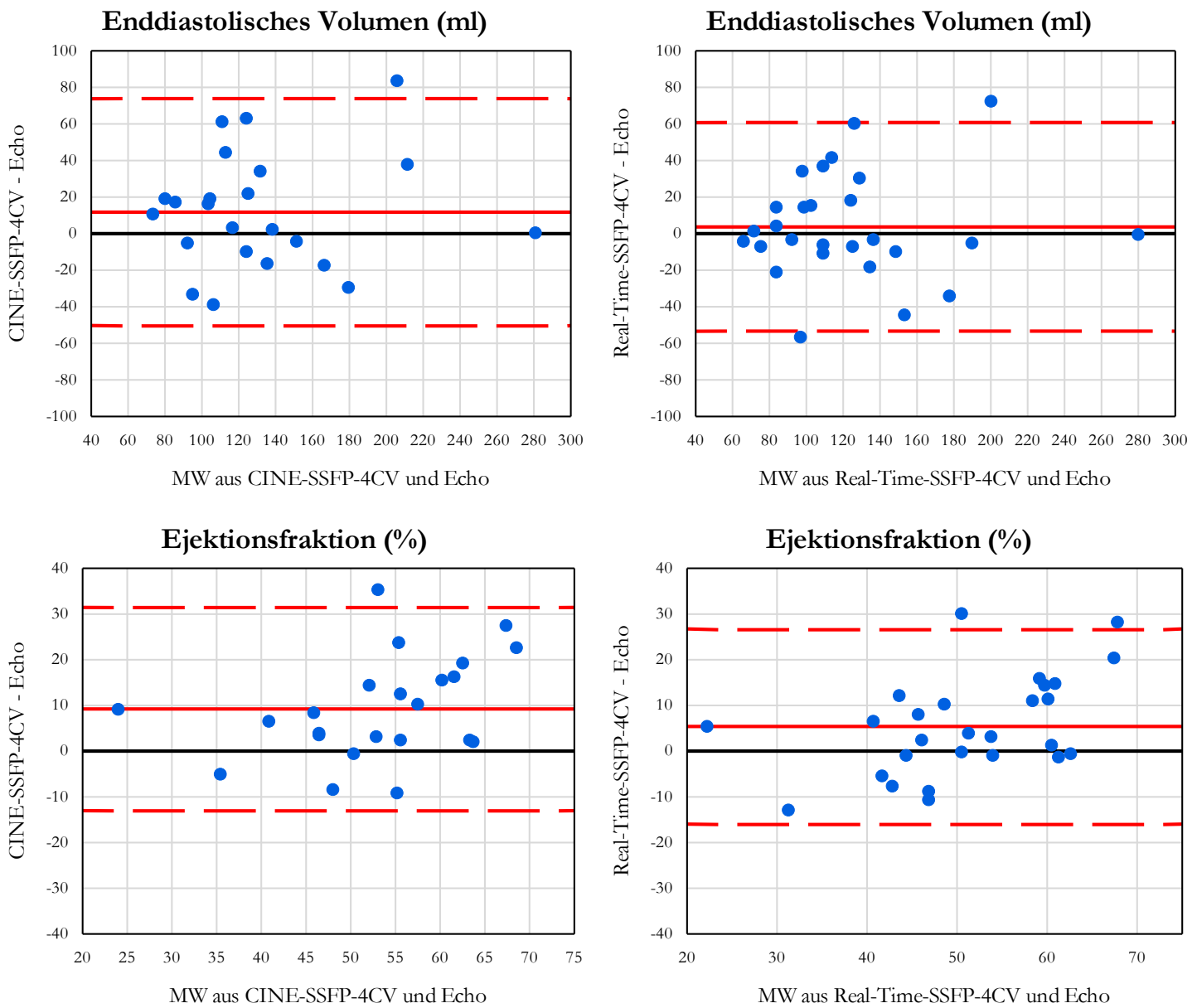

Abbildung 26: Bland-Altman-Plots zum Vergleich zwischen CINE-SSFP-4CV und Echokardiographie (links) sowie Real-Time-SSFP und Echokardiographie (rechts) für EDV und EF. Echo $=$ Echokardiographie

\subsubsection{Vergleich der Mittelung über sieben, fünf und drei Schläge des Real-Time- SSFP-4CV}

Bei 29 der 30 untersuchten Patienten lag ein segmentierbarer Real-Time-SSFP-4CV vor. Die Mittelung aus sieben Schlägen wurde nun mit der Mittelung aus fünf bzw. drei Schlägen verglichen. In der Bland-Altman-Analyse wurde der Wert der Mittelung über drei oder über fünf Schläge jeweils vom Mittelwert über sieben Schläge abgezogen.

Sowohl für den Vergleich mit der Mittelung über fünf als auch über drei Schläge ergaben sich nur sehr geringe Verzerrungen für alle erhobenen Parameter. Der Vergleich mit der Mittelung über drei Schläge weist nur für einige Werte eine geringfügig höhere Verzerrung auf, jedoch liegen die Übereinstimmungsgrenzen hier etwas weiter auseinander. Die BlandAltman-Analysen für EDV und EF sind in Tabelle 19 dargestellt. Abbildung 27 zeigt die zugehörigen Plots. 
Tabelle 19: Bland-Altman-Analyse - Vergleich der funktionellen Analyse des Real-Time-SSFP4CV über sieben, fünf und drei Schläge; $\mathrm{n}=29$ von 30 .

\begin{tabular}{|c|c|c|c|}
\hline \multicolumn{4}{|c|}{ Vergleich 7 vs. 5 Stapel } \\
\hline Parameter & Verzerrung & & \\
\hline MM (g) & 0 & -7 & 7 \\
\hline EDV (ml) & -2 & -10 & 6 \\
\hline ESV (ml) & -1 & -5 & 3 \\
\hline $\mathrm{SV}(\mathrm{ml})$ & -1 & -8 & 6 \\
\hline $\mathrm{EF}(\%)$ & 0 & -3 & 4 \\
\hline $\mathrm{HZV}(1 / \mathrm{min})$ & 0 & $-0,6$ & 0,6 \\
\hline \multicolumn{4}{|c|}{ Vergleich 7 vs. 3 Stapel } \\
\hline Parameter & Verzerrung & & \\
\hline MM (g) & 0 & -13 & 12 \\
\hline EDV (ml) & -3 & -19 & 14 \\
\hline $\mathrm{ESV}(\mathrm{ml})$ & -2 & -11 & 7 \\
\hline $\mathrm{SV}(\mathrm{ml})$ & -1 & -13 & 12 \\
\hline $\mathrm{EF}(\%)$ & 1 & -6 & 7 \\
\hline $\mathrm{HZV}(1 / \mathrm{min})$ & $-0,1$ & $-1,3$ & 1,1 \\
\hline
\end{tabular}



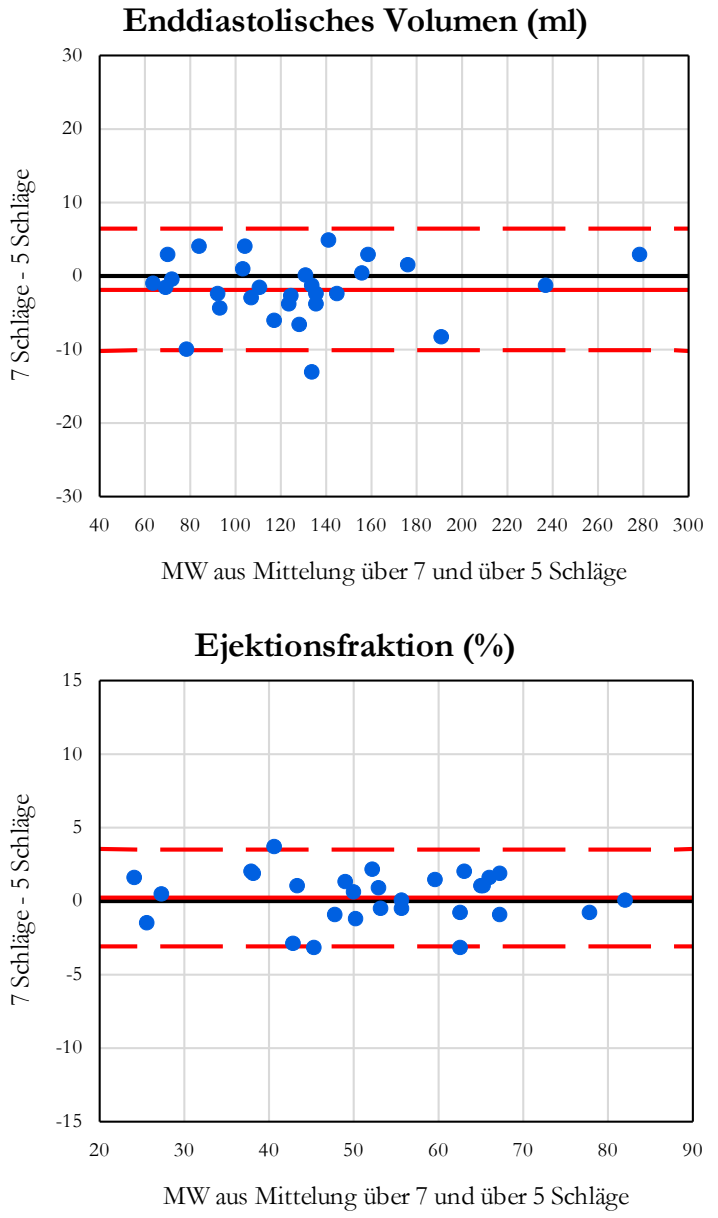
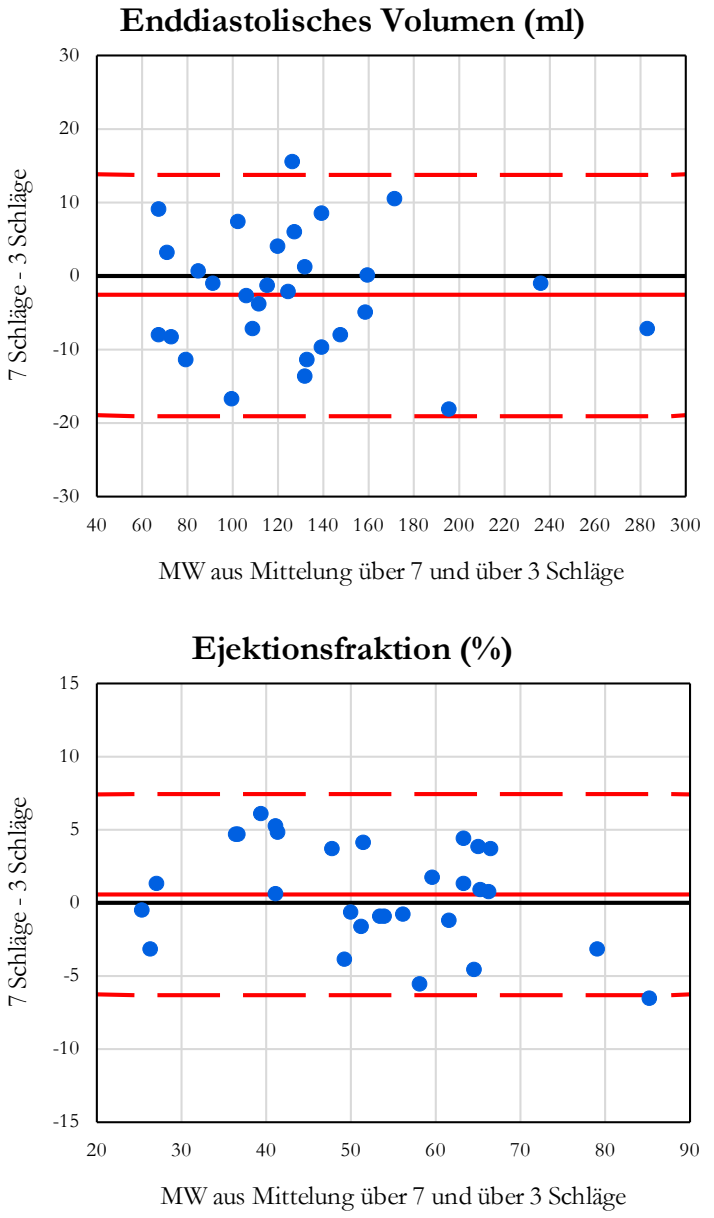

Abbildung 27: Bland-Altman-Plots für den Vergleich der Mittelung über sieben Schläge mit der Mittelung über fünf Schläge (links) und drei Schläge (rechts) des Real-Time-SSFP-4CV für EDV und EF.

\subsubsection{Schlag-zu-Schlag-Analyse im Real-Time-SSFP-4CV}

Für die Schlag-zu-Schlag-Analyse, die eine Aussage über die Schwankung der EF des Einzelpatienten über mehrere Schläge ermöglicht, wurden im Real-Time-SSFP-4CV bei 29 Patienten je sieben Schläge analysiert. Die mittlere Spannweite der Schlagdauer (Differenz zwischen längstem und kürzestem Schlag) betrug $477 \mathrm{~ms}$ bei einer Standardabweichung von 365 ms. Die Spannweite der EF pro Patient lag im Mittel bei $23 \pm 10 \%$. In Abbildung 28 ist die Spannweite der EF für jeden Patienten graphisch dargestellt. 


\section{Spannweite der EF im Real-Time-SSFP-4CV}

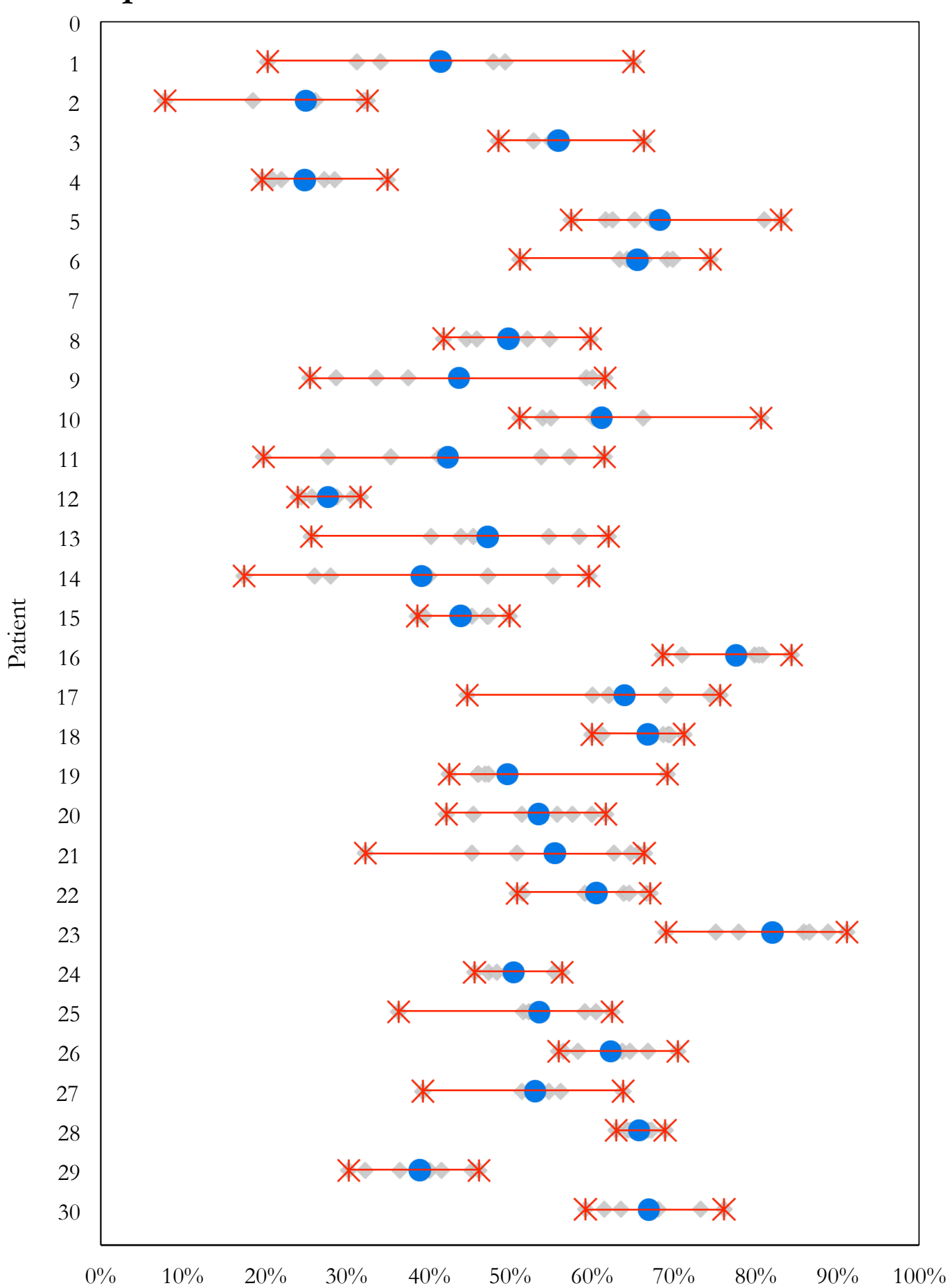

Ejektionsfraktion (\%)

EF des Einzelschlages $\quad$ Mittelwert $\quad *$ Minimum $\quad *$ Maximum

Abbildung 28: Spannweite der EF bei allen Patienten über sieben Schläge. Für jeden Schlag konnten eigene funktionelle Parameter erhoben werden. 


\section{Diskussion}

Ziel dieser Arbeit war das Evaluieren einer neuen Echtzeit-MRT hinsichtlich der Bildqualität und Eignung zur Analyse der linksventrikulären Funktion bei Patienten mit VHF im Vergleich mit der üblicherweise in der kardialen MRT angewandten CINE-SSFP. Diese kann aufgrund der in Kapitel 1.5 beschriebenen Limitationen nur als Vergleichs-, nicht aber als Referenzmethode verwendet werden. Dem übergeordnet ist die Frage, inwieweit die Real-Time-SSFP grundsätzlich für die Herzdiagnostik bei Patienten mit Herzrhythmusstörungen geeignet ist. Die Bildqualität der CINE-SSFP ist bei diesen Patienten oft reduziert (Contijoch et al. 2015). Beide Methoden wurden hinsichtlich diagnostischer Bildqualität und Eignung zur Analyse der linksventrikulären Funktion miteinander verglichen. Als ergänzende Vergleichsmethode für die funktionellen Parameter diente die Echokardiographie.

Verschiedene Verfahren sind für die Echtzeit-Bildgebung in der kardialen MRT entwickelt worden, wobei meist die Beschleunigung der Datenakquisition durch eine Unterabtastung der Bildinformation erzielt wird, was mit einer Reduktion der räumlichen Auflösung verbunden ist (Contijoch et al. 2015). Die meisten dieser Methoden benötigen vor der Bildakquisition eine Kalibrierung, die einen steady-state der Magnetisierung herstellt. Die Unterabtastung kann kartesisch, spiral oder radial erfolgen. Einige Studien haben gezeigt, dass radiale Verfahren - wie auch in dieser Studie verwendet - den anderen hinsichtlich Bildqualtät und Eignung zur funktionellen Analyse überlegen sind (Muthurangu et al. 2008; Spuentrup et al. 2003). Dass die Real-Time-MRT zuverlässige Ergebnisse bei der Analyse der Herzfunktion im Sinusrhythmus liefern kann, wurde mehrfach gezeigt (Kuhl et al. 2004; Nagel et al. 2000). Die Vorzüge der Nutzung der Real-Time-MRT für Patienten mit Arrhythmien wurden bereits erkannt (Contijoch et al. 2015; Zhang et al. 2014). Der Vorteil der hier verwendeten Real-Time-Technik liegt in einer guten räumlichen Auflösung trotz starker Unterabtastung, die durch die effiziente Bildrekonstruktion mit nichtlinearer Inversion zustande kommt (Uecker et al. 2010a). Inzwischen gelingt dies nicht nur bei einer zeitlichen Auflösung von 40 ms; auch eine noch höhere zeitliche Auflösung von 20 bis $30 \mathrm{~ms}$ ist bereits möglich (Uecker et al. 2010b). Außerdem ist keinerlei Kalibrierung erforderlich und Bildserien können ohne Pause konsekutiv aufgenommen werden. Damit können Bildfolgen vom Herzschlag in Echtzeit gewonnen werden, was eine Betrachtung des Einzelschlages möglich macht und viele weitere im Folgenden beschriebene Vorteile bietet:

Bei der Echtzeit-MRT sind im Gegensatz zur CINE-MRT keine Atemkommandos mehr nötig. Der Ablauf der Untersuchung wird dadurch für Personal und Patienten erleichtert. Die Aufnahmen können EKG-unabhängig gewonnen werden, wodurch es nicht zu Artefakten aufgrund eines gestörten EKG-Signals kommt. Durch die schnellere 
Datenakquisition, den Wegfall der Atemkommandos und der EKG-Triggerung ergibt sich eine erhebliche Zeitersparnis. Dies ist insbesondere für die Untersuchung von Kindern, multimorbiden Patienten oder Patienten mit Klaustrophobie sowie aus wirtschaftlicher Sicht wichtig.

Viele der genannten Studien zur kardialen Echtzeit-Bildgebung wurden auf Basis einer SSFP-Sequenz durchgeführt. Diese gilt wegen des guten Signal-zu-Rausch-Verhältnisses und des guten Kontrastes zwischen Myokard und Blutpool als Methode der Wahl in der kardialen MRT (Kramer et al. 2013), wobei in der Regel mit einer Feldstärke von 1,5 Tesla gearbeitet wird. 93,6\% der Kardio-MRT-Untersuchungen in Europa finden bei einer Feldstärke von 1,5 Tesla statt (Bruder et al. 2013). Die in dieser Studie verwendete Echtzeitmethode wurde ursprünglich für die T1-gewichtete FLASH-Sequenz auf einem 3Tesla-Gerät entwickelt. Voit et al. (2013) haben gezeigt, dass diese Form der Datenakquisition und Bildberechnung auch bei der SSFP-Sequenz und einer Feldstärke von 1,5 Tesla funktioniert; für gesunde Probanden und Patienten mit verschiedenen Herzerkrankungen liefert sie sehr ähnliche Werte für die funktionellen Parameter. Daher war es sinnvoll, die Untersuchung an Patienten mit VHF ebenfalls an einem 1,5-TeslaGerät auf Basis der SSFP-Sequenz durchzuführen. Auch Contijoch et al. (2015) führten aktuell ihre Real-Time-MRT-Studie an einem 1,5-Tesla-Gerät durch. Da die neue Echtzeitmethode vollständig auf bereits etablierten Techniken basiert und mit Ausnahme der Installation des Bildrechners keine Veränderungen an der Hardware vorgenommen werden müssen, ist es leicht möglich, sie zur breiten Anwendung zur Verfügung zu stellen.

Die Segmentation fand manuell statt, wobei die CINE-SSFP-Aufnahmen in Anlehnung an die aktuellen Leitlinien segmentiert wurden (Schulz-Menger et al. 2013). Für einen SAStapel waren pro Patient je nach Herzgröße etwa 12 bis 24 Bilder zu segmentieren. Der Zeitaufwand entsprach ungefähr dem in der klinischen Routine. Durch die bei vielen Arrhythmikern reduzierte Bildqualität war die Konturfindung allerdings deutlich erschwert. Weiterhin war problematisch, dass beim automatischen Zusammensetzen des SA-Stapels ED und ES oft nicht korrekt untereinandergelegt wurden. In diesem Falle wurden ED und ES in einer der midventrikulären Schichten festgelegt. Anschließend wurden alle Bilder derselben Spalte segmentiert. Dies führte zu einem Fehler in der Berechnung der Volumina, der methodenbedingt nicht vermeidbar ist und gelegentlich auch bei Patienten im Sinusrhythmus auftritt.

Die Konturabgrenzung gelang in vielen Fällen in der Real-Time-SSFP deutlich leichter. Jedoch war der Segmentationsaufwand dadurch erhöht, dass ED und ES in jeder Schicht neu festgelegt werden mussten. Ein automatisches Untereinanderlegen der zueinander passenden Herzphasen über alle Schichten erfolgte durch die zur Verfügung stehende Software nicht. Bei der Echtzeitakquisition wäre das aufgrund der unterschiedlichen Schlaglängen auch nicht möglich. Die Sortierung musste also manuell erfolgen. In dieser Studie wurden sieben konsekutive Stapel segmentiert, um - wie in der CINE - über 
ausreichend viele Schläge Mittelwerte bilden zu können. Dies erforderte die Segmentation von 84 bis 168 Bildern pro Patient. Die Ergebnisse (siehe Kapitel 3.3.3) haben gezeigt, dass auch weniger Schläge genügen, um zuverlässige Werte für die funktionellen Parameter zu erhalten. Dennoch bleibt die Segmentation der Echtzeitaufnahmen wegen der aufwendigen Sortierung der Herzphasen mit einem erhöhten Arbeitsaufwand verbunden. Um die neue Echtzeitmethode gut in klinische Arbeitsabläufe zu integrieren, ist also eine Weiterentwicklung der Segmentationssoftware, insbesondere der automatischen Segmentation, nötig, wie bereits Zhang et al. (2014) angemerkt haben. Eine gute automatische Segmentation würde auch das Evaluieren der Füllungsdynamik ermöglichen, da dann nicht mehr nur einzelne Herzphasen, sondern ganze Serien segmentiert werden könnten. Vielversprechende Ansätze zur automatischen Konturerkennung bei Real-TimeBildserien, die mit der hier verwendeten Bildberechnungsmethode gewonnen wurden, gibt es bereits für Flussmessungen (Chitiboi et al. 2014). Auch für die funktionelle Analyse geeignete Software ist in Arbeit (Chitiboi et al. 2015).

Da die Echtzeit-Datenakquisition bei hoher zeitlicher Auflösung noch nicht dreidimensional erfolgen kann, ist ein Real-Time-SSFP-SA-Stapel immer eine Mittelung über mehrere Schläge, denn die Schichten werden nacheinander aufgenommen. Das bedeutet, dass zwar die Datenakquisition in Echtzeit erfolgt, eine 3D-Echtzeitvolumetrie allerdings noch nicht möglich ist. Echtzeitvolumetrie gelingt bisher nur zweidimensional, also z.B. aus einer SASchicht oder mit der Flächen-Längen-Methode aus dem 4CV. Erst wenn die dreidimensionale Bildakquisition gelingt, wird die 3D-Echtzeitvolumetrie möglich sein.

\subsection{Qualitätsanalyse}

Die Bewertung der Bildqualität anhand einer Notenskala erfolgte auch in anderen Studien zum Vergleich der Real-Time-MRT mit der CINE-MRT, so z.B. bei Muthurangu et al. (2008) oder Spuentrup et al. (2003). In der hier diskutierten Studie wurde eine Likert-Skala in Anlehnung an Voit et al. (2013) verwendet. Die Qualitätsanalyse hat gezeigt, dass die Real-Time-SSFP bei beiden Untersuchern in der Bewertung besser abschneidet. Im Gesamteindruck erhielt sie mit Ausnahme des SA apex bei beiden Untersuchern signifikant bessere Bewertungen. In der Studie von Voit et al. (2013) erhielten die Real-Time-SSFPAufnahmen im Mittel eine leicht schlechtere Bewertung. Dort wurden allerdings vorwiegend Probanden und Patienten im Sinusrhythmus untersucht. Bei ihnen ist zu erwarten, dass in der CINE die Bildqualität höher liegt, da bei den über mehrere Sekunden akquirierten CINE-Daten mehr Information zur Bildberechnung zur Verfügung steht. Durch die Unterabtastung kann es in der Real-Time-SSFP zu Streifenartefakten kommen, die die Bildqualität zusätzlich herabsetzen (Block et al. 2007; Zhang et al. 2010). Dennoch waren auch bei Patienten im Sinusrhythmus die Real-Time-Aufnahmen diagnostisch gut verwertbar. Voit et al. (2013) untersuchten in ihrer Studie lediglich drei Patienten mit Arrhythmien. Dabei stellten sie eine deutlich bessere Bildqualität bei der Real-Time-SSFP 
als bei der CINE-SSFP fest. In der hier vorliegenden, daran anschließenden Studie kann dieses zunächst als Tendenz gewertete Ergebnis bestätigt werden.

Wie einleitend beschrieben (siehe Kapitel 1.5), kann die EKG-abhängige CINE-SSFP unter VHF schnell versagen, weil die Synchronisierung der CINE-Daten durch den irregulären Rhythmus misslingt, was zu Artefakten führt (Bassett et al. 2014). Durch retrospektives Aussortieren von Schlägen mit stark abweichender Dauer bei der Berechnung der CINE-SSFP-Aufnahmen wird versucht, dieses Problem zu umgehen. Allerdings werden diese Schläge dann nicht mehr bei der funktionellen Analyse berücksichtigt, obwohl sie ebenfalls Teil der Herzfunktion sind. Bei deutlicher Arrhythmie versagt diese Sortierung außerdem, da alle Schläge voneinander abweichen. Weiterhin ist die CINE-SSFP für EKG-Signal- und atembedingte Artefakte anfällig. Dies alles erklärt die schlechtere Bildqualität der CINE-SSFP, deren Bilder in einigen Fällen nicht für die funktionelle Analyse verwendbar waren.

Da die hier verwendete Real-Time-SSFP EKG-unabhängig während normaler Atmung funktioniert und keine Interpolation der Daten über mehrere Schläge erfolgt, ist die Bildqualität trotz der Limitationen meist signifikant höher als die der CINE-SSFP. Der Vorteil macht sich besonders bei Patienten mit stark ausgeprägter Arrhythmie der einzelnen Herzschläge bemerkbar, zeigt sich aber auch bei Patienten mit weniger ausgeprägter Arrhythmie.

SA apex wurde insgesamt schlechter bewertet als die anderen Aufnahmen. Nur bei einem der beiden Untersucher schneidet hier die Real-Time signifikant besser ab. Es kann vermutet werden, dass die Strukturunterscheidung durch den besonders kleinen Blutpool und die Bewegung der Spins, die zu Phasendifferenzen führt, erschwert ist. Aus den so entstandenen Phasendifferenzen kann sich eine Unschärfe ergeben (McRobbie et al. 2006).

Was für die Bewertung des Gesamteindrucks gilt, gilt auch für einen Großteil der Einzelkriterien: In den meisten Fällen schneidet die Real-Time-SSFP in allen Blicken mit Ausnahme von SA apex besser ab. Hinsichtlich Abgrenzbarkeit der Papillarmuskeln wird die Real-Time-Sequenz nur von einem Untersucher als signifikant besser bewertet. Die Papillarmuskeln sind für die Bestimmung von Masse und Volumina deutlich weniger von Bedeutung als die Abgrenzbarkeit der großen Strukturen Septum und Seitenwand. Bei einigen Segmentationsprogrammen ist ihre Segmentation gar nicht möglich (SchulzMenger et al. 2013).

Es fällt auf, dass Untersucher 2 viele Blicke hinsichtlich Gesamteindruck sowie der Einzelkriterien signifikant besser bewertete als Untersucher 1. Wie bei jedem visuellen Scoring-System fließen also auch hier die Untersuchererfahrung und individuelle Bewertungsschwerpunkte in das Ergebnis ein.

Die überwiegend bessere Bewertung der Real-Time-SSFP im Gesamteindruck und hinsichtlich Einzelkriterien durch beide Untersucher zeigt das Potenzial der Technik bei 
Patienten mit VHF, wobei die scharfe Darstellung des Apex nach wie vor problematisch ist.

\subsection{Linksventrikuläre Funktionsparameter}

Zur funktionellen Analyse wurden nur Aufnahmen mit ausreichend guter Bildqualität herangezogen, da bei den übrigen keine sinnvolle Segmentation möglich war. Von 30 CINE-SSFP-SA-Stapeln waren vier nicht auswertbar; dies entspricht 13,33\%. In der RealTime-SSFP erfolgten keine Ausschlüsse wegen schlechter Bildqualität. Sechs von 30 CINE-SSFP-4CV mussten ausgeschlossen werden (20\%), während nur ein Real-TimeSSFP-4CV nicht auswertbar war (3,33\%). Die Real-Time-SSFP liefert also mit deutlich höherer Sicherheit Bilddaten, die zur funktionellen Analyse herangezogen werden können.

Mit Bland-Altman-Plots wurden die Ergebnisse der CINE-SSFP mit denen der Real-TimeSSFP verglichen. Sowohl im SA-Stapel als auch im 4CV ist die Verzerrung in einem diagnostisch akzeptablen Bereich. Es besteht eine klare Tendenz der Real-Time-SSFP, kleinere Werte für die funktionellen Parameter zu messen. Auffällig sind die weiten Übereinstimmungsgrenzen, die sich durch einige Ausreißer erklären lassen. Für die aus dem SA-Stapel bestimmte EF beispielsweise misst die Real-Time-SSFP in zwei Fällen deutlich höhere Werte, was auch erklärt, warum im Gegensatz zu allen anderen Parametern für die EF im Mittel etwas höhere Werte mit der Real-Time-SSFP angegeben werden. Dies kann dadurch zustande gekommen sein, dass durch starke Artefakte in der CINE-SSFP und eine ungenaue Sortierung der Schichten enddiastolisch und endsystolisch die Volumetrie erschwert war. Möglicherweise erschien das Myokard durch die Unschärfe enddiastolisch breiter, sodass der Blutpool unterschätzt wurde und dadurch die Ejektionsfraktion erniedrigt erschien.

Die CINE-SSFP-Aufnahmen wurden unter Atemanhalt gewonnen, während die RealTime-SSFP-Aufnahmen unter freier Atmung akquiriert wurden. Dadurch entsteht ein Unterschied in den physiologischen Parametern, der zu Differenzen zwischen den Aufnahmemodalitäten führt. Allerdings wurden bereits bei Voit et al. (2013) in einer Studie, die vorwiegend Patienten im Sinusrhythmus einschloss, mit der Real-Time-SSFP tendenziell niedrigere Werte gemessen. Die Unterschätzung der Werte durch die RealTime-SSFP bei Patienten mit VHF kommt möglicherweise dadurch zustande, dass bei dieser Methode konsekutiv jede Ventrikelkontraktion einbezogen wurde und dadurch auch Schläge mit besonders schlechter Auswurfleistung in die Auswertung einflossen. Bei der CINE-SSFP werden einige Schläge durch die Arrhythmie-Korrektur (siehe Kapitel 1.5) außenvorgelassen und die Herzfunktion erscheint besser. Auch die besonders arrhythmischen Schläge sind aber Bestandteil der Herzfunktion des Patienten mit VHF, sodass die Ergebnisse der Real-Time-SSFP tatsächlich realitätsnäher sind.

Der Vergleich mit der Echokardiographie wurde nur im 4CV durchgeführt. Vergleiche der 2D-Echokardiographie mit der kardialen MRT (CINE) haben schon vielfach vorher 
stattgefunden (Bellenger et al. 2000; R Hoffmann et al. 2005; Mistry et al. 2010). In diesen Studien hatten sich nur kleine Differenzen zwischen den mit MRT und Echokardiographie gemessenen Werten ergeben; EDV und ESV wurden durch die Echokardiographie in allen Studien unterschätzt (Wood et al. 2014). In der vorliegenden Studie zeigte sich, dass die Echokardiographie die EF deutlicher als in anderen Studien als kleiner bewertet, wohingegen diese Tendenz für das EDV weniger ausgeprägt war und das ESV sogar leicht größer gemessen wurde. Insgesamt war die Tendenz zur „Unterschätzung“ der Werte durch die Echokardiographie aber auch in dieser Studie zu beobachten. Die Verzerrung von EDV, SV, EF und HZV war beim Vergleich mit der Real-Time-SSFP etwas geringer als beim Vergleich mit der CINE-SSFP und auch die Streuung war für die meisten Parameter geringer. Dennoch fällt bei beiden Vergleichen die breite Streuung der Werte ins Auge. Alle Echokardiographien wurden von einem erfahrenen Untersucher durchgeführt, um eine Interobserver-Variabilität zu vermeiden. Die 2D-Echokardiographie bleibt trotzdem ein eher untersucherabhängiges Verfahren mit höherer Inter- und IntraobserverVariabilität (R Hoffmann et al. 2005). Auch dadurch kann die breite Streuung erklärt werden.

Zusammenfassend kann festgestellt werden, dass die Real-Time-SSFP dadurch, dass deutlich weniger Aufnahmen wegen schlechter Bildqualität ausgeschlossen werden mussten, für die Analyse der Herzfunktion bei Patienten mit VHF die zuverlässigere Technik als die CINE-SSFP ist. Die Tendenz der Real-Time, die CINE hinsichtlich Volumina und Ejektionsfraktion zu unterschätzen, ist deutlich erkennbar. Weil in der Auswertung der Real-Time-SSFP keine Arrhythmie-Korrektur vorgenommen wurde, wurden auch Schläge mit kürzerer Schlagdauer und eher schlechterer Auswurfleistung berücksichtigt, weshalb die Werte der Real-Time-SSFP hier plausibler sind. Außerdem sprechen die Ergebnisse der Qualitätsanalyse sowie der Vergleich mit der Echokardiographie dafür, dass die Real-Time-SSFP die zuverlässigere Messung ist. Dass die Verzerrung zwischen CINE und Real-Time nicht allzu groß ist, ist positiv, obgleich die CINE-SSFP bei Arrhythmikern aufgrund der eingeschränkten Bildqualität nicht als Goldstandard betrachtet werden kann. Vielmehr kann aus den Ergebnissen geschlossen werden, dass die CINE-SSFP bei weniger ausgeprägter Arrhythmie und diagnostischer Bildqualität auch zuverlässig segmentiert werden kann.

\subsubsection{Vergleich der Mittelung über sieben, fünf und drei Schläge des Real-Time- SSFP-4CV}

Sowohl für den Vergleich mit fünf Schlägen als auch mit drei Schlägen ergab sich eine sehr geringe Verzerrung: Für die EF lag sie unter einem Prozent. Die Spannweite der Übereinstimmungsgrenzen ist beim Vergleich mit drei Schlägen etwas breiter. Auch eine Segmentation von weniger Schlägen liefert also zuverlässige Werte für die Herzfunktion, wobei eine Segmentation von fünf Schlägen genauer erscheint. Ähnlich wie in der Echokardiographie könnte bei Verwendung der Real-Time-SSFP je nach Ausprägung der 
Arrhythmie und Bildqualität entschieden werden, wie viele Schläge aufgenommen und analysiert werden. Diese Flexibilität ist ein Vorteil der Real-Time-MRT. Eine Reduktion auf weniger Schläge führt zu einer Verkürzung der Akquisitions- und Nachanalysezeit. Außerdem reduziert sich dadurch die Menge an Bilddaten, was Datentransfer und Datenspeicherung erleichtert.

\subsubsection{Schlag-zu-Schlag-Analyse im Real-Time-SSFP-4CV}

Im Real-Time-SSFP-4CV war eine Schlag-zu-Schlag-Analyse der linksventrikulären Funktion mit der Flächen-Längen-Methode möglich. Die Leitlinien empfehlen, die linksventrikuläre Funktion aus dem SA-Stapel zu erheben. Die Analyse aus dem 4CV ist ebenso anerkannt, solange keine wesentlichen Wandbewegungsstörungen zu erwarten sind (Schulz-Menger et al. 2013). Derzeit ist eine Schlag-zu-Schlag-Analyse nur zweidimensional möglich, da das gleichzeitige Akquirieren mehrerer Schichten noch nicht gelingt.

Die EF konnte für jeden Einzelschlag erhoben werden. Von jedem Patienten wurden sieben Schläge betrachtet und es ergab sich eine mittlere Spannweite der EF von $23 \pm 10 \%$ Standardabweichung. Nach ausführlicher Literaturrecherche konnten nur wenige Arbeiten gefunden werden, die sich mit der Funktion des Einzelschlages bei Patienten mit VHF befasst haben. Schneider et al. (1983) ermittelten die Ejektionsfraktion mit einer nuklearmedizinischen Methode unter Verwendung von Technetium-99m. Die funktionellen Parameter wurden über 50 konsekutive Schläge ermittelt und eine über alle Patienten gepoolte Standardabweichung der EF von 6,9\% bei Patienten mit VHF errechnet. Die Analyse der Schlag-zu-Schlag-Variation bei Patienten mit VHF war nicht der Schwerpunkt dieser Studie. Die durchgeführte Auswertung vermochte dennoch zu zeigen, dass eine Evaluation des Einzelschlages in der MRT möglich ist. Um wirklich aussagekräftige Ergebnisse zu erhalten, ist allerdings die dreidimensionale Datenakquisition zur Darstellung des ganzen Ventrikels erforderlich.

\subsection{Fazit}

Die Studie hat gezeigt, dass die Real-Time-SSFP im Vergleich zur CINE-SSFP bei Patienten mit VHF eine höhere Bildqualität aufweist. Das Bildmaterial ist mit höherer Zuverlässigkeit für die funktionelle Analyse geeignet, wobei für Masse und Volumina mit der Real-Time-SSFP tendenziell kleinere Werte gemessen werden. Der Vergleich mit der Echokardiographie zeigte bei der Real-Time-SSFP eine etwas geringere Verzerrung. Für eine zuverlässige Aussage scheint die Segmentation von drei bis fünf Herzschlägen in der Real-Time-SSFP zu genügen, wobei die Mittelung über fünf Schläge genauer ist. Dies bedeutet eine erhebliche Zeitersparnis in der Nachverarbeitung und eine Reduktion der Datenmenge. Durch die Echtzeit-Datenakquisition ist bereits eine Schlag-zu-SchlagAnalyse zweidimensional möglich. Weitere Studien, die die klinische Relevanz der Schlagzu-Schlag-Analyse evaluieren, sollten folgen. Für eine reelle Echtzeit-3D-Volumetrie ist 
allerdings eine dreidimensionale Echtzeit-Datenakquisition erforderlich - eine Herausforderung der MRT-Forschung und der Nachverarbeitungslösungen in den nächsten Jahren. Problematisch sind die großen Datenmengen, die durch die EchtzeitBildgebung entstehen, und die damit verbundene lange Nachbearbeitungszeit. SoftwareEntwicklungen mit dem Ziel des schnelleren Datentransfers und der vollautomatischen Segmentation sind unbedingt erforderlich, um die Real-Time-Sequenz, die schon eine gute diagnostische Bildqualität aufweist, in den klinischen Arbeitsalltag integrieren zu können. $\mathrm{Da}$ an vielen Instituten auch heute noch 1,5-Tesla-Geräte in der klinischen Routine verwendet werden, war es wichtig, zu zeigen, dass Real-Time-MRT mit radialer Unterabtastung und Rekonstruktion durch NLINV auch bei einem 1,5-Tesla-Gerät diagnostisch verwertbare Bilder liefert. Um dem Trend zur höheren Feldstärke gerecht zu werden, sollten weitere Studien bei einer Feldstärke von 3 Tesla erfolgen, auch unter der Fragestellung, ob SSFP- oder FLASH-Sequenz besser geeignet sind. Über die Analyse der linksventrikulären Funktion hinaus kann nun die Eignung der Real-Time-Sequenz für andere Anwendungen der Herzbildgebung bei VHF wie z.B. Fluss oder Perfusion evaluiert werden. Die Ergebnisse dieser Studie zeigen, dass die vollständige MRT-Herzuntersuchung in Echtzeit EKG-unabhängig und ohne Atemkommandos auch bei Patienten mit Arrhythmien möglich ist. 


\section{$5 \quad$ Zusammenfassung}

Die kardiale MRT gilt als „Goldstandard“ zur linksventrikulären Funktionsanalyse. Üblicherweise wird hierfür die CINE-SSFP verwendet, die auf einer Interpolation der Bilddaten über mehrere Herzschläge beruht. Dieses Verfahren ist gut geeignet für Patienten im Sinusrhythmus. Bei Arrhythmien gerät es an seine Grenzen, da die Zuordnung der Rohdaten zur richtigen Herzphase erschwert ist. Ein neues Echtzeitverfahren mit einer zeitlichen Auflösung von $40 \mathrm{~ms}$ (Real-Time-SSFP) kann konsekutiv Bilder von der Herzbewegung aufnehmen.

30 Patienten mit Vorhofflimmern (VHF) zum Zeitpunkt der Untersuchung wurden mit der CINE-SSFP und mit der Real-Time-SSFP untersucht. Zusätzlich erhielten sie eine transthorakale Echokardiographie. Sowohl die CINE- als auch die Real-Time-Bilder wurden von zwei Untersuchern hinsichtlich der Diagnosequalität beurteilt. Aus beiden Aufnahmemodalitäten wurden die funktionellen Parameter mit einer gängigen Segmentationssoftware erhoben. Es erfolgte ein Vergleich der Bildqualität und der funktionellen Parameter. Außerdem wurden sowohl die CINE- als auch die Real-TimeWerte mit der Echokardiographie verglichen. Real-Time-SSFP-Aufnahmen ermöglichten die zweidimensionale Schlag-zu-Schlag-Analyse mit Angabe der Spannweite der Ejektionsfraktion.

Bei der Analyse der Diagnosequalität schnitt die Real-Time-SSFP deutlich besser ab. Mehr als zehn Prozent der CINE-Aufnahmen konnten wegen schlechter Bildqualität nicht funktionell ausgewertet werden. Beim Vergleich der funktionellen Werte ergab sich ein Unterschied zwischen den Methoden im diagnostisch akzeptablen Bereich, wobei die RealTime-SSFP tendenziell niedrigere Werte maß. Der Vergleich mit der Echokardiographie ergab für die Real-Time-SSFP meist eine etwas geringere mittlere Abweichung und Streuung als für die CINE-SSFP.

Die Studie hat gezeigt, dass es mit der Real-Time-SSFP möglich ist, diagnostisch hochwertige MRT-Bilder von Patienten mit VHF zu generieren. Auch in der funktionellen Analyse zeigt sie sich gegenüber der CINE-SSFP überlegen. Die Weiterentwicklung der Segmentationssoftware für die klinische Anwendung hat bereits begonnen. Eine echte 3DVolumetrie bedarf der 3D-Datenakquisition - aktuell eine große Herausforderung der MRT-Forschung. Weitere Einsatzmöglichkeiten der Real-Time-Sequenz bei Arrhythmiepatienten können nun evaluiert werden. 


\section{$6 \quad$ Literaturverzeichnis}

Attili AK, Schuster A, Nagel E, Reiber JH, van der Geest RJ (2010): Quantification in cardiac MRI: advances in image acquisition and processing. Int J Cardiovasc Imaging 26 Suppl 1, 27-40

Bassett EC, Kholmovski EG, Wilson BD, DiBella EV, Dosdall DJ, Ranjan R, McGann CJ, Kim D (2014): Evaluation of highly accelerated real-time cardiac cine MRI in tachycardia. NMR Biomed 27, 175-182

Bauer RW, Radtke I, Block KT, Larson MC, Kerl JM, Hammerstingl R, Graf TG, Vogl TJ, Zhang S (2013): True real-time cardiac MRI in free breathing without ECG synchronization using a novel sequence with radial $\mathrm{k}$-space sampling and balanced SSFP contrast mode. Int J Cardiovasc Imaging 29, 1059-1067

Bellenger NG, Burgess MI, Ray SG, Lahiri A, Coats AJ, Cleland JG, Pennell DJ (2000): Comparison of left ventricular ejection fraction and volumes in heart failure by echocardiography, radionuclide ventriculography and cardiovascular magnetic resonance; are they interchangeable? Eur Heart J 21, 1387-1396

Bland JM, Altman DG (1999): Measuring agreement in method comparison studies Stat Methods. Med Res 8, 135-160

Bloch F (1946): Nuclear Induction. Phys Rev 70, 460-474

Block KT, Uecker M, Frahm J (2007): Undersampled radial MRI with multiple coils. Iterative image reconstruction using a total variation constraint. Magn Reson Med 57, 1086-1098

Bluemke DA, Boxerman JL, Atalar E, McVeigh ER (1997): Segmented K-space cine breath-hold cardiovascular MR imaging: Part 1. Principles and technique. Am J Roentgenol 169, 395-400

Bruder O, Wagner A, Lombardi M, Schwitter J, van Rossum A, Pilz G, Nothnagel D, Steen H, Petersen S, Nagel E, et al. (2013): European Cardiovascular Magnetic Resonance (EuroCMR) registry--multi national results from 57 centers in 15 countries. J Cardiovasc Magn Reson 15, 9

Buck T, Breithardt O-A, Faber L, Fehske W, Flachskampf F, Franke A, Hagendorff A, Hoffmann R, Kruck I, Kücherer H et al. (2009): Manual zur Indikation und Durchführung der Echokardiographie. Clin Res Cardiol Suppl 51, 3-51

Carr HY (1958): Steady-State Free Precession in Nuclear Magnetic Resonance. Phys Rev 112, 1693-1701

Chapman B, Turner R, Ordidge RJ, Doyle M, Cawley M, Coxon R, Glover P, Mansfield P (1987): Real-time movie imaging from a single cardiac cycle by NMR. Magn Reson Med 5, 246-254

Chitiboi T, Hennemuth A, Tautz L, Huellebrand M, Frahm J, Linsen L, Hahn H (2014): Context-based segmentation and analysis of multi-cycle real-time cardiac MRI. IEEE Int Symp Biomed Imaging 11, 943-946

Chitiboi T, Hennemuth A, Tautz L, Huellebrand M, Frahm J, Hahn H (2015): Automatic multi-cycle analysis of cardiac functionfrom real-time MRI. J Cardiovasc Magn Reson 17, 385 
Contijoch F, Witschey WR, Rogers K, Rears H, Hansen M, Yushkevich P, Gorman J, 3rd, Gorman RC, Han Y (2015): User-initialized active contour segmentation and golden-angle real-time cardiovascular magnetic resonance enable accurate assessment of LV function in patients with sinus rhythm and arrhythmias. J Cardiovasc Magn Reson 17, 37

Dewey M, Muller M, Eddicks S, Schnapauff D, Teige F, Rutsch W, Borges AC, Hamm B (2006): Evaluation of global and regional left ventricular function with 16-slice computed tomography, biplane cineventriculography, and two-dimensional transthoracic echocardiography: comparison with magnetic resonance imaging. J Am Coll Cardiol 48, 2034-2044

Du Bois D, Du Bois EF (1916): Clinical calorimetry: tenth paper a formula to estimate the approximate surface area if height and weight be known. Arch Intern Med 17, 863871

Edler I, Lindstrom K (2004): The history of echocardiography. Ultrasound Med Biol 30, 1565-1644

Evangelista A, Flachskampf F, Lancellotti P, Badano L, Aguilar R, Monaghan M, Zamorano J, Nihoyannopoulos P, European Association of E (2008): European Association of Echocardiography recommendations for standardization of performance, digital storage and reporting of echocardiographic studies. Eur J Echocardiogr 9 , 438-448

Fasshauer M, Joseph AA, Kowallick JT, Unterberg-Buchwald C, Merboldt KD, Voit D, Steinmetz M, Staab W, Schaetz S, Zhang S, et al. (2014): Real-time phase-contrast flow MRI of haemodynamic changes in the ascending aorta and superior vena cava during Mueller manoeuvre. Clin Radiol 69, 1066-1071

Friedrich MG, Marcotte F (2013): Cardiac magnetic resonance assessment of myocarditis. Circ Cardiovasc Imaging $\underline{6}$, 833-839

Fuchs F, Laub G, Othomo K (2003): TrueFISP--technical considerations and cardiovascular applications. Eur J Radiol 46, 28-32

Go AS, Hylek EM, Phillips KA, Chang Y, Henault LE, Selby JV, Singer DE (2001): Prevalence of diagnosed atrial fibrillation in adults: national implications for rhythm management and stroke prevention: the AnTicoagulation and Risk Factors in Atrial Fibrillation (ATRIA) Study. JAMA 285, 2370-2375

Goldman MR, Pohost GM, Ingwall JS, Fossel ET (1980): Nuclear magnetic resonance imaging: potential cardiac applications. Am J Cardiol 46, 1278-1283

Haase A, Frahm J, Matthaei D, Hanicke W, Merboldt KD (1986): Flash Imaging - Rapid Nmr Imaging Using Low Flip-Angle Pulses. J Magn Reson 67, 258-266

Hawkes RC, Holland GN, Moore WS, Roebuck EJ, Worthington BS (1981): Nuclear magnetic resonance (NMR) tomography of the normal heart. J Comput Assist Tomogr $\underline{5}, 605-612$

Hoffmann KT, Hosten N, Ehrenstein T, Gutberlet M, Roricht S, Felix R (2000): [The T2weighted half-Fourier acquired single-shot turbo-spin-echo technic compared to the conventional T2-weighted turbo-spin-echo technic for cerebral magnetic resonance tomography. A sequence comparison]. Rofo 172, 521-526 
Hoffmann R, von Bardeleben S, ten Cate F, Borges AC, Kasprzak J, Firschke C, Lafitte S, Al-Saadi N, Kuntz-Hehner S, Engelhardt M et al. (2005): Assessment of systolic left ventricular function: a multi-centre comparison of cineventriculography, cardiac magnetic resonance imaging, unenhanced and contrast-enhanced echocardiography. Eur Heart J 26, 607-616

Joseph A, Kowallick JT, Merboldt KD, Voit D, Schaetz S, Zhang S, Sohns JM, Lotz J, Frahm J (2014): Real-time flow MRI of the aorta at a resolution of $40 \mathrm{msec}$. J Magn Reson Imaging 40, 206-213

Kellman P, Chefd'hotel C, Lorenz CH, Mancini C, Arai AE, McVeigh ER (2009): High spatial and temporal resolution cardiac cine MRI from retrospective reconstruction of data acquired in real time using motion correction and resorting. Magn Reson Med 62, 1557-1564

Kino A, Zuehlsdorff S, Sheehan JJ, Weale PJ, Carroll TJ, Jerecic R, Carr JC (2009): Threedimensional phase-sensitive inversion-recovery turbo FLASH sequence for the evaluation of left ventricular myocardial scar. Am J Roentgenol 193, W381-388

Kowallick JT, Joseph AA, Unterberg-Buchwald C, Fasshauer M, van Wijk K, Merboldt KD, Voit D, Frahm J, Lotz J, Sohns JM (2014): Real-time phase-contrast flow MRI of the ascending aorta and superior vena cava as a function of intrathoracic pressure (Valsalva manoeuvre). Br J Radiol 87, 20140401

Kramer CM, Barkhausen J, Flamm SD, Kim RJ, Nagel E, Society for Cardiovascular Magnetic Resonance Board of Trustees Task Force on Standardized P (2013): Standardized cardiovascular magnetic resonance (CMR) protocols 2013 update. J Cardiovasc Magn Reson 15, 91

Kuhl HP, Spuentrup E, Wall A, Franke A, Schroder J, Heussen N, Hanrath P, Gunther RW, Buecker A (2004): Assessment of myocardial function with interactive nonbreath-hold real-time MR imaging: comparison with echocardiography and breathhold Cine MR imaging. Radiology 231, 198-207

Lang RM, Bierig M, Devereux RB, Flachskampf FA, Foster E, Pellikka PA, Picard MH, Roman MJ, Seward J, Shanewise JS, et al. (2005): Recommendations for chamber quantification: a report from the American Society of Echocardiography's Guidelines and Standards Committee and the Chamber Quantification Writing Group, developed in conjunction with the European Association of Echocardiography, a branch of the European Society of Cardiology. J Am Soc Echocardiogr 18, 1440-1463

Lauterbur PC (1973): Image Formation by Induced Local Interactions: Examples Employing Nuclear Magnetic Resonance. Nature 242, 190-191

Lehmann EL, D’Abrera H: Nonparametrics: Statistical Methods Based on Ranks (rev. ed.) Prentice-Hall.Springer Verlag, New York 1998

Lenz GW, Haacke EM, White RD (1989): Retrospective cardiac gating: a review of technical aspects and future directions. Magn Reson Imaging $\underline{7}, 445-455$

Leschka S, Scheffel H, Desbiolles L, Plass A, Gaemperli O, Valenta I, Husmann L, Flohr TG, Genoni M, Marincek B (2007): Image quality and reconstruction intervals of dual-source CT coronary angiography: recommendations for ECG-pulsing windowing. Invest Radiol 42, 543-549

Likert R (1932): A technique for the measurement of attitudes. Arch Psychol 22 140, 55

Mansfield P, Maudsley A (1977): Medical imaging by NMR. Br J Radiol 50, 188-194 
McRobbie DW, Moore EA, Graves MJ, Prince MR: MRI from Picture to Proton. Cambridge university press, Cambridge 2006

Mistry N, Halvorsen S, Hoffmann P, Muller C, Bohmer E, Kjeldsen SE, Bjornerheim R (2010): Assessment of left ventricular function with magnetic resonance imaging vs. echocardiography, contrast echocardiography, and single-photon emission computed tomography in patients with recent ST-elevation myocardial infarction. Eur J Echocardiogr 11, 793-800

Moon JC, Lorenz CH, Francis JM, Smith GC, Pennell DJ (2002): Breath-hold FLASH and FISP cardiovascular MR imaging: left ventricular volume differences and reproducibility. Radiology 223, 789-797

Muthurangu V, Lurz P, Critchely JD, Deanfield JE, Taylor AM, Hansen MS (2008): Realtime assessment of right and left ventricular volumes and function in patients with congenital heart disease by using high spatiotemporal resolution radial k-t SENSE. Radiology 248, 782-791

Nagel E, Schneider U, Schalla S, Ibrahim T, Schnackenburg B, Bornstedt A, Klein C, Lehmkuhl HB, Fleck E (2000): Magnetic resonance real-time imaging for the evaluation of left ventricular function. J Cardiovasc Magn Reson 2, 7-14

Nijm GM, Swiryn S, Larson AC, Sahakian AV (2008): Extraction of the magnetohydrodynamic blood flow potential from the surface electrocardiogram in magnetic resonance imaging. Med Biol Eng Comput 46, 729-733

O’Donnell T, Funka-Lea G, Tek H, Jolly M-P, Rasch M, Setser R (2006): Comprehensive cardiovascular image analysis using MR and CT at Siemens Corporate Research. Int J Comput Vis $\underline{70}, 165-178$

Olthoff A, Zhang S, Schweizer R, Frahm J (2014): On the physiology of normal swallowing as revealed by magnetic resonance imaging in real time. Gastroenterol Res Pract 2014, 493174

Omoto R (1982): Echocardiographic evaluation of left ventricular size, shape and functionadvantages and limitations of this method. Jpn Circ J 46, 1121-1126

Petitjean C, Dacher J-N (2011): A review of segmentation methods in short axis cardiac MR images. Med Image Anal 15, 169-184

Petrov G, Kelle S, Fleck E, Wellnhofer E (2015): Incremental cost-effectiveness of dobutamine stress cardiac magnetic resonance imaging in patients at intermediate risk for coronary artery disease. Clin Res Cardiol 104, 401-409

Picano E (2005): Economic and biological costs of cardiac imaging. Cardiovasc Ultrasound $\underline{3}, 13$

Purcell EM, Torrey HC, Pound RV (1946): Resonance Absorption by Nuclear Magnetic Moments in a Solid. Phys Rev 69, 37-38

Rauschenberg J: Optimierte Pulssequenzen für die interventionelle Magnetresonanztomographie mit einem robotischen Assistenzsystem. Dissertation. Naturwissenschaftlich-Mathematische Gesamtfakultät der Ruprecht-Karls-Universität Heidelberg. 2008

Ridgway JP (2010): Cardiovascular magnetic resonance physics for clinicians: part I. J Cardiovasc Magn Reson 12, 71

Rzedzian RR, Pykett IL (1987): Instant images of the human heart using a new, wholebody MR imaging system. Am J Roentgenol 149, 245-250 
Saeed M, Van TA, Krug R, Hetts SW, Wilson MW (2015): Cardiac MR imaging: current status and future direction. Cardiovasc Diagn Ther $\underline{5}, 290-310$

Scheffler K, Lehnhardt S (2003): Principles and applications of balanced SSFP techniques. Eur Radiol 13, 2409-2418

Schneider J, Berger HJ, Sands MJ, Lachman AB, Zaret BL (1983): Beat-to-beat left ventricular performance in atrial fibrillation: radionuclide assessment with the computerized nuclear probe. Am J Cardiol 51, 1189-1195

Schulz-Menger J, Bluemke DA, Bremerich J, Flamm SD, Fogel MA, Friedrich MG, Kim RJ, von Knobelsdorff-Brenkenhoff F, Kramer CM, Pennell DJ, et al. (2013): Standardized image interpretation and post processing in cardiovascular magnetic resonance: Society for Cardiovascular Magnetic Resonance (SCMR) board of trustees task force on standardized post processing. J Cardiovasc Magn Reson 15, 35

Siadecki SD, Frasure SE, Lewiss RE, Saul T (2015): High Body Mass Index is Strongly Correlated with Decreased Image Quality in Focused Bedside Echocardiography. J Emerg Med 50.2, 295-301

Spuentrup E, Schroeder J, Mahnken AH, Schaeffter T, Botnar RM, Kuhl HP, Hanrath P, Gunther RW, Buecker A (2003): Quantitative assessment of left ventricular function with interactive real-time spiral and radial MR imaging. Radiology 227, 870-876

Stewart S, Hart CL, Hole DJ, McMurray JJ (2001): Population prevalence, incidence, and predictors of atrial fibrillation in the Renfrew/Paisley study. Heart 86, 516-521

Uecker M, Zhang S, Frahm J (2010a): Nonlinear inverse reconstruction for real-time MRI of the human heart using undersampled radial FLASH. Magn Reson Med 63, 14561462

Uecker M, Zhang S, Voit D, Karaus A, Merboldt KD, Frahm J (2010b): Real-time MRI at a resolution of 20 ms. NMR Biomed 23, 986-994

van der Geest RJ, Jansen E, Buller V, Reiber J: Automated detection of left ventricular epiand endocardial contours in short-axis MR images. IEEE 1994, 33-36

Voit D, Zhang S, Unterberg-Buchwald C, Sohns JM, Lotz J, Frahm J (2013): Real-time cardiovascular magnetic resonance at $1.5 \mathrm{~T}$ using balanced SSFP and $40 \mathrm{~ms}$ resolution. J Cardiovasc Magn Reson 15, 79

Wood PW, Choy JB, Nanda NC, Becher H (2014): Left ventricular ejection fraction and volumes: it depends on the imaging method. Echocardiography 31, 87-100

Xue H, Kellman P, Larocca G, Arai AE, Hansen MS (2013): High spatial and temporal resolution retrospective cine cardiovascular magnetic resonance from shortened free breathing real-time acquisitions. J Cardiovasc Magn Reson 15, 102

Zhang S: Real-time magnetic resonance imaging. Ph.D. Diss., Göttingen 2009

Zhang S, Uecker M, Voit D, Merboldt KD, Frahm J (2010): Real-time cardiovascular magnetic resonance at high temporal resolution: radial FLASH with nonlinear inverse reconstruction. J Cardiovasc Magn Reson 12, 39

Zhang S, Joseph AA, Voit D, Schaetz S, Merboldt KD, Unterberg-Buchwald C, Hennemuth A, Lotz J, Frahm J (2014): Real-time magnetic resonance imaging of cardiac function and flow-recent progress. Quant Imaging Med Surg 4, 313-329 
Zhang S, Joseph AA, Gross L, Ghadimi M, Frahm J, Beham AW (2015): Diagnosis of Gastroesophageal Reflux Disease Using Real-time Magnetic Resonance Imaging. Sci Rep $\underline{5}, 12112$

Zhou X, Thavendiranathan P, Chen Y, Cheng L, Qian Z, Liu S, Houle H, Zhi G, Vannan MA (2015): Feasibility of Automated Three-Dimensional Rotational Mechanics by Real-Time Volume Transthoracic Echocardiography: Preliminary Accuracy and Reproducibility Data Compared with Cardiovascular Magnetic Resonance. J Am Soc Echocardiogr 29(1), 62-73 


\section{Danksagung}

Mein Dank gilt allen, die mich bei der Erstellung dieser Dissertation unterstützt haben. Im Einzelnen sind zu nennen:

- Prof. Dr. med. Joachim Lotz - Als Abteilungsleiter hat er die Durchführung der Studie angeregt und jederzeit mit Expertise und Innovation gefördert.

- Prof. Dr. med. Christina Unterberg-Buchwald - Als erste Berichterstatterin hat sie die Dissertation von Anfang an begleitet, als kardiologische Oberärztin alle Echokardiographien durchgeführt und die Qualitätsbeurteilung der MRT-Bilder selbst vorgenommen.

- PD Dr. med. Jan-Martin Sohns - Der Betreuer war insbesondere an der Ausarbeitung der Qualitätsanalyse und der Segmentationsprozesse beteiligt. Er hat ebenfalls alle MRT-Bilder qualitativ beurteilt.

- Das Herzzentrum der UMG unter Leitung von Prof. Dr. Gerd Hasenfuß - Aus dieser Klinik wurden die Studienpatienten rekrutiert.

- Die Biomedizinische NMR Forschungs GmbH aus Göttingen unter Leitung von Prof. Dr. rer. nat. Jens Frahm - Dort wurde das Echtzeitverfahren entwickelt, mit dem hier gearbeitet wurde. Während der Durchführung der Studie stand das Team mit Rat und Tat zur Seite.

- Das Deutsche Zentrum für Herz-Kreislauf-Forschung e.V., das mich während der Studienarbeit finanziell unterstützte. 


\section{Lebenslauf}

Geboren wurde ich am 31.08.1990 in Kassel als Sohn von Elisabeth Hornschuh-von Loesch (staatlich geprüfte Betriebswirtin) und Eckhart von Loesch (Berufsreitlehrer FN).

Von 1997 bis 2001 besuchte ich die Grundschule in Immenhausen-Holzhausen und wechselte anschließend bis 2003 auf die Freiherr-vom-Stein-Schule (Gesamtschule) in Immenhausen. Von 2003 bis 2010 war ich Schüler der Albert-Schweitzer-Schule (Gymnasium) in Kassel. Hier legte ich mein Abitur ab und erwarb das Zertifikat „Level 2 English for Business (Reading and Writing)“ der London Chamber of Commerce and Industry.

Den Zivildienst leistete ich in der Zeit vom 01.07.2010 bis zum 31.12.2010 beim ArbeiterSamariter-Bund (ASB), Ortsverband Kassel, im Bereich soziale Dienste ab.

Am 01.04.2011 begann ich das Studium der Humanmedizin an der Universitätsmedizin Göttingen. Im März 2013 bestand ich den Ersten Abschnitt der Ärztlichen Prüfung. Seit dem 01.04.2013 studiere ich im klinischen Studienabschnitt an der UMG. Von Oktober 2013 bis März 2014 arbeitete ich im Rahmen eines Freisemesters ausschließlich an dieser Dissertation, wobei ich vom Deutschen Zentrum für Herz-Kreislauf-Forschung e.V. finanziell unterstützt wurde. Weiterhin erhielt ich vom 01.04.2014 bis zum 31.09.2014 das „Gö4Med“-Stipendium der Universitätsmedizin Göttingen für semesterbegleitendes wissenschaftliches Arbeiten. Seit Oktober 2014 arbeite ich als hilfswissenschaftlicher Mitarbeiter am Institut für Diagnostische- und Interventionelle Radiologie. 